\title{
CHARACTERISTIC CLASSES VIA 4-DIMENSIONAL GAUGE THEORY
}

\author{
HOKUTO KONNO
}

\begin{abstract}
We construct characteristic classes of 4-manifold bundles using $S O(3)$-Yang-Mills theory and Seiberg-Witten theory for families.
\end{abstract}

\section{Contents}

1. Introduction

2. Structure groups

3. ASD setting and SW setting

4. Cocycle condition modulo gauge

5. Virtual neighborhood for families

5.1. Virtual neighborhood

5.2. Family virtual neighborhood

6. Construction of the characteristic classes $\quad 19$

6.1. Main construction 20

6.2. Well-definedness and naturality 23

7. Characteristic classes as obstruction 27

8. Calculations 28

8.1. Almost localized harmonic forms 29

8.2. $\quad$ SW for connected sum with $n\left(S^{2} \times S^{2}\right)$

8.3. Behavior under the composition $1 \quad 41$

8.4. Behavior under the composition 2

8.5. Ruberman's calculation and $\mathbb{D} \quad 45$

9. Concluding remarks 46

References 46

\section{INTRODUCTION}

The aim of this paper is to construct characteristic classes for bundles whose fiber is a 4-manifold using gauge theory. Our construction can be regarded as an infinite dimensional analogue of that of familiar characteristic classes typified by the Mumford-Morita-Miller classes [29 31] for surface bundles. To explain it, let us recall the definition of the Mumford-Morita-Miller classes. From a topological point of view, the definition of these classes includes the following two steps:

(1): Consider a "linearization" of a given surface bundle, more precisely, the vector bundle consisting of tangent vectors along the fibers.

(2): Consider the Euler class of the linearization. 
The Mumford-Morita-Miller classes are defined as the fiber integration of cup products of the Euler class in (2). On the other hand, there are the following infinite dimensional analogues of (1) and (2):

(I): Consider a functional space on a given a non-linear object, say a manifold. (II): Consider the zero set of a given Fredholm section of a Hilbert bundle.

The reason why (I) and (II) correspond to (1) and (2) can be explained as follows. On (I), a functional space on a given manifold is also regarded as a "linearization" of the manifold. For example, in representation theory, given a manifold acted by a Lie group, the induced infinite dimensional representation on a functional space on the manifold can be regarded as a linearization of the original non-linear action. We also note that, if the non-linear object is given as a fiber bundle, one can consider a family of functional spaces as its linearization. On (II), suppose that we are given an infinite dimensional Hilbert bundle on a Hilbert manifold defined as some functional spaces on a finite dimensional manifold and that we are also given a Fredholm section of it. The zero set of the section morally corresponds to the "Euler class" of the infinite dimensional bundle. We recall that (II) is one of the main ideas to define the invariants of 4-manifolds emerging from gauge theory, say the Donaldson invariant [8] and the Seiberg-Witten invariant [43. In this paper, using gauge theory, we shall construct characteristic classes for bundles of 4-manifolds via the above analogy between (1), (2) and (I), (II).

The initial roots of this work have been given by S. K. Donaldson [7,9. He has suggested the idea of characteristic classes based on family gauge theory, however there are several problems to justify the idea. The first one is the natural structure groups which are compatible with gauge theoretic equations are not subgroups of the diffeomorphism group of the 4-manifold given as a fiber. (For example, see M. Szymik [41.) The second problem arises if we want to establish the universal theory: the base space of the universal bundle of the diffeomorphism group, namely the classifying space of the diffeomorphism group, is not a finite dimensional smooth manifold. Since the usual family gauge theory is considered on smooth finite dimensional parameter spaces, it cannot be applied to this situation. In this paper we use an idea given by N. Nakamura [33] and a family version of Y. Ruan's virtual neighborhood technique 37] to overcome these difficulties of the construction. In general, even if we could succeed to construct a characteristic class of bundles of smooth manifolds, it is a difficult problem to prove that the characteristic class is non-trivial. (For example, in the case of the Mumford-Morita-Miller classes, the proof of the non-triviality is one of difficult parts of S. Morita 30.) However, for our characteristic classes, we can use D. Ruberman's results [38] and author's [20] on invariants of diffeomorphisms, and they ensure that our characteristic classes are non-trivial. We also give other calculations in Section 8

We now explain our main results. We can construct our theory of characteristic classes using both the $S O(3)$-Yang-Mills anti-self-dual (ASD) equation and the Seiberg-Witten equations. Let $X$ be an oriented closed smooth 4-manifold, $\operatorname{Diff}^{+}(X)$ be the group of orientation preserving diffeomorphisms on $X, \mathfrak{P}$ be the isomorphism class of an $S O(3)$-bundle on $X$ with $w_{2}(\mathfrak{P}) \neq 0$, and $\mathfrak{s}$ be the isomorphism class of a $\operatorname{spin}^{c}$ structure on $X$. (Although an $S O(3)$ )-bundle (or a $\operatorname{spin}^{c}$ structure) and the isomorphism class of it are often denoted by a same notation, we explicitly distinguish them in this paper.) We define subgroups $\operatorname{Diff}(X, \mathfrak{P})$ and 
$\operatorname{Diff}(X, \mathfrak{s})$ of $\operatorname{Diff}^{+}(X)$ by

$$
\begin{aligned}
& \operatorname{Diff}(X, \mathfrak{P}):=\left\{f \in \operatorname{Diff}^{+}(X) \mid f^{*} \mathfrak{P}=\mathfrak{P}\right\}, \text { and } \\
& \operatorname{Diff}(X, \mathfrak{s}):=\left\{f \in \operatorname{Diff}^{+}(X) \mid f^{*} \mathfrak{s}=\mathfrak{s}\right\} .
\end{aligned}
$$

Let $\mathcal{O}$ be a homology orientation of $X$ (i.e. an orientation of the vector space $H^{1}(X ; \mathbb{R}) \oplus H^{+}(X ; \mathbb{R})$, where $H^{+}(X ; \mathbb{R})$ is a maximal positive definite subspace of $H^{2}(X ; \mathbb{R})$ with respect to the intersection form of $\left.X\right)$. We define

$$
\begin{aligned}
& \operatorname{Diff}(X, \mathfrak{P}, \mathcal{O}):=\left\{f \in \operatorname{Diff}(X, \mathfrak{P}) \mid f^{*} \mathcal{O}=\mathcal{O}\right\}, \text { and } \\
& \operatorname{Diff}(X, \mathfrak{s}, \mathcal{O}):=\left\{f \in \operatorname{Diff}(X, \mathfrak{s}) \mid f^{*} \mathcal{O}=\mathcal{O}\right\} .
\end{aligned}
$$

We denote by $d(\mathfrak{P})$ and $d(\mathfrak{s})$ the formal dimension of the moduli space of the $S O(3)$ ASD equations for $\mathfrak{P}$ and the Seiberg-Witten equations for $\mathfrak{s}$ respectively: given by the formula $d(\mathfrak{P})=-2 p_{1}(\mathfrak{P})-3\left(1-b_{1}(X)+b^{+}(X)\right)$ and $d(\mathfrak{s})=\left(c_{1}(\mathfrak{s})^{2}-2 \chi(X)-\right.$ $3 \operatorname{sign}(X)) / 4$. We shall construct characteristic classes of bundles of a 4-manifold $X$ with structure group $\operatorname{Diff}(X, \mathfrak{P}), \operatorname{Diff}(X, \mathfrak{P}, \mathcal{O})$ and $\operatorname{Diff}(X, \mathfrak{s}), \operatorname{Diff}(X, \mathfrak{s}, \mathcal{O})$ using families of ASD equations and Seiberg-Witten equations respectively, summarized as follows:

Theorem 1.1. Let $n$ be a non-negative integer satisfying that $b^{+}(X) \geq n+2$.

(1) Assume that $d(\mathfrak{P})=-n$. Then we can give cohomology classes

$$
\mathbb{D}(X, \mathfrak{P}) \in H^{n}(B \operatorname{Diff}(X, \mathfrak{P}) ; \mathbb{Z} / 2)
$$

and

$$
\mathbb{D}(X, \mathfrak{P}, \mathcal{O}) \in H^{n}(B \operatorname{Diff}(X, \mathfrak{P}, \mathcal{O}) ; \mathbb{Z})
$$

via n-parameter families of $S O(3)$-Yang-Mills ASD equations. These cohomology classes depend only on the pair $(X, \mathfrak{P})$ and the triple $(X, \mathfrak{P}, \mathcal{O})$ respectively.

(2) Assume that $d(\mathfrak{s})=-n$. Then we can give cohomology classes

$$
\mathbb{S W}(X, \mathfrak{s}) \in H^{n}(B \operatorname{Diff}(X, \mathfrak{s}) ; \mathbb{Z} / 2)
$$

and

$$
\mathbb{S W}(X, \mathfrak{s}, \mathcal{O}) \in H^{n}(B \operatorname{Diff}(X, \mathfrak{s}, \mathcal{O}) ; \mathbb{Z})
$$

via n-parameter families of Seiberg-Witten equations. These cohomology classes depend only on the pair $(X, \mathfrak{s})$ and the triple $(X, \mathfrak{s}, \mathcal{O})$ respectively.

See Definition 6.10 for the corresponding statement. The cases that $n=0$ in (1) and (2) of Theorem 1.1 are nothing other than the mod 2 and the usual $S O(3)$ Donaldson invariants of $(X, \mathfrak{P})$ and Seiberg-Witten invariants of $(X, \mathfrak{s})$ respectively, via $H^{0}(B \operatorname{Diff}(X, \mathfrak{P}) ; \mathbb{Z} / 2) \cong \mathbb{Z} / 2$ and $H^{0}(B \operatorname{Diff}(X, \mathfrak{P}, \mathcal{O}) ; \mathbb{Z}) \cong \mathbb{Z}$, and similar isomorphisms for $\mathfrak{s}$.

As usual, Theorem 1.1 can be rephrased as follows. For $n \geq 0$ and a CW complex $B$, if we are given a bundle $X \rightarrow E \rightarrow B$ whose structure group $G$ is given as (1), (22), (3), or (4), we can functorially define a cohomology class $\mathbb{D}(E) \in H^{n}(B)$ or $\mathbb{S W}(E) \in H^{n}(B)$ under suitable assumptions on $b^{+}$and the formal dimension. This cohomology class is given as the pull-back of the universal characteristic class given in Theorem 1.1. (In fact our characteristic classes are defined even if $B$ is a general topological space. See Theorem 6.9 for the summarized statements.) If $n>0$ and the characteristic class $\mathbb{D}$ or $\mathbb{S W}$ of $E$ is non-trivial, then we can see that $E$ is a non-trivial $G$-bundle, explained in Remark 6.5. We mention that non-vanishing 
of our characteristic classes implies indecomposability as fiberwise connected sum under certain conditions, and $\mathbb{S W}$ also obstructs the existence of a family of positive scalar curvature metrics, described in Section 7.

Since it is hard to compute gauge theoretic invariants in general, one may wonder computability of our characteristic classes. In particular, it is natural to ask whether there are non-trivial examples for a positive degree $n>0$. In Section 8 we shall give several non-trivial calculations of our characteristic classes for $n>0$. As well as the usual Donaldson invariants and Seiberg-Witten invariants, SW is relatively easy to compute rather than $\mathbb{D}$. We here note that, if $X$ is simply connected and $\mathfrak{s}$ is the isomorphism class of a spin ${ }^{c}$ structure coming from a spin structure on $X$, then we have $\operatorname{Diff}(X, \mathfrak{s})=\operatorname{Diff}^{+}(X)$, and thus we can get a cohomology class on $B \operatorname{Diff}^{+}(X)$ by considering $\mathbb{S W}$. For example, denoting by $\mathfrak{s}$ the isomorphism class of a $\operatorname{spin}^{c}$ structure coming from a spin structure on $K 3 \# n\left(S^{2} \times S^{2}\right)$ for a positive integer $n$, we see that

$$
\mathbb{S W}\left(K 3 \# n\left(S^{2} \times S^{2}\right), \mathfrak{s}\right) \neq 0 \text { in } H^{n}\left(B \operatorname{Diff}^{+}\left(K 3 \# n\left(S^{2} \times S^{2}\right)\right) ; \mathbb{Z} / 2\right),
$$

which is explained in Example 8.10. We also mention that $\mathbb{S W}$ can detect a difference between $\operatorname{Homeo}(X, \mathfrak{s})$-bundles and $\operatorname{Diff}(X, \mathfrak{s})$-bundles. Here $\operatorname{Homeo}(X, \mathfrak{s})$ is the group of homeomorphisms which preserve the orientation and $\mathfrak{s}$, as in the definition of $\operatorname{Diff}(X, \mathfrak{s})$. For example, for some natural numbers $k, l>0$, we can find the isomorphism class $\mathfrak{s}$ of a $\operatorname{spin}^{c}$ structure on $X=k \mathbb{C P}^{2} \# l\left(-\mathbb{C P}^{2}\right)$ and a bundle $X \rightarrow E \rightarrow S^{1}$ with structure group $\operatorname{Diff}(X, \mathfrak{s})$ such that $E$ is trivial as a $\operatorname{Homeo}(X, \mathfrak{s})$-bundle, but non-trivial as a $\operatorname{Diff}(X, \mathfrak{s})$-bundle, deduced by showing $\mathbb{S W}(E) \neq 0$. See Example 8.19, This argument is a Seiberg-Witten analogue of one in D. Ruberman 38.

We note that family gauge theory has been studied by several people: 18, 20, $22,25,32,33,38,41$. The relation between some of these works and this paper is as follows. (See also Section 9]) D. Ruberman [38 40] has given invariants of diffeomorphisms, and the author generalized a part of 38] to a tuple of commutative diffeomorphisms in 20]. Our characteristic classes can be regarded as a generalization of the invariants of (tuples of) diffeomorphisms given in [20,38]. T.-J. Li and A.-K. Liu [25] and M. Szymik [41] have considered families of $\operatorname{spin}^{c}$ 4-manifolds and Seiberg-Witten equations. In addition, Szymik has considered characteristic cohomotopy classes for bundles of $\operatorname{spin}^{c} 4$-manifolds using a finite dimensional approximation of Seiberg-Witten equations, in other words, family version of BauerFuruta invariant [5]. (See Remark 9.2.) One of the big differences between theories of [25, 41] and our theory is the structure group: the structure group of their family is the automorphism group of a given $\operatorname{spin}^{c} 4$-manifold. This group does not coincide with our structure group $\operatorname{Diff}(X, \mathfrak{s})$ (or $\operatorname{Diff}(X, \mathfrak{s}, \mathcal{O})$ ). This is because $\operatorname{Diff}(X, \mathfrak{s})$ respects only the isomorphism class of a $\operatorname{spin}^{c}$ structure. As we mentioned, this is the first difficulty to construct a theory of characteristic classes whose structure group is (a subgroup of) the diffeomorphism group, and we use an idea of Nakamura 33 to deal with it. In Section 8, to calculate our characteristic classes we consider a higher-dimensional generalization of an argument given in D. Ruberman [38 40] and the idea due to N. Nakamura [33] on double mapping tori as in 20]. Kronheimer [22] has considered a homological formulation of family gauge theory to study the space of symplectic forms of a 4-manifold, and the author [19] has considered a cohomological formulation of family gauge theory to study the adjunction inequality. One of the important differences between [19, 22, and the 
current paper is a type of families. Families of perturbations or Riemannian metrics of a fixed 4-manifold are considered in [19,22, and they are therefore trivial families as a family of 4-manifolds. On the other hand, in this paper we consider families of 4-manifolds themselves, and our characteristic classes can detect the non-triviality as families of 4-manifolds, as we explained.

We also note that, in symplectic geometry, several people have studied characteristic classes of symplectic fibrations based on the study of a family version of Gromov-Witten invariant. (See H.-V. Lê and K. Ono [24], O. Buşe [6], and D. McDuff [27.) Our theory of characteristic classes may be regarded as the gauge theoretic counterpart of their work.

The author hopes that this paper will be the first of a series of a cohomological study of family gauge theory, which was also considered in [22] and [19] from different points of view as we explained. Further potential developments hoped to be given in subsequent papers include a Bauer-Furuta version of our characteristic classes, calculations relating to algebraic geometry, and Floer homology analogue of this paper. (See Section 9 for details of some of them.)

Acknowledgement. The author would like to express his deep gratitude to Mikio Furuta for the helpful suggestions and for continuous encouragement during this work. The author would also like to express his appreciation to Yosuke Morita for asking him about fiberwise connected sum formula. It induces the author to consider Theorem 7.1. The author also wishes to thank David Baraglia for pointing out some mistakes and giving comments for a preprint version of this paper. The author was supported by JSPS KAKENHI Grant Number 16J05569 and the Program for Leading Graduate Schools, MEXT, Japan.

\section{Structure Groups}

In this section we define the structure groups for our characteristic classes and some related groups. We first consider Yang-Mills theory. To construct a theory of characteristic classes fruitfully, we need some compactness, corresponding to Assumptions 5.1 and 5.8. We therefore consider $S O(3)$-Yang-Mills ASD equations rather than $S U(2)$-Yang-Mills ASD equations. Let $X$ be an oriented smooth 4manifold, $P \rightarrow X$ be an $S O(3)$-bundle, and $\mathfrak{P}$ be the isomorphism class of $P$. We have already defined the groups $\operatorname{Diff}(X, \mathfrak{P})$ and $\operatorname{Diff}(X, \mathfrak{P}, \mathcal{O})$ in the introduction. We here define

$$
\operatorname{Aut}(X, P):=\left\{\begin{array}{l|c}
(f, \tilde{f}) & \begin{array}{c}
f \in \operatorname{Diff}(X, \mathfrak{P}), \\
\tilde{f}: P \rightarrow P \text { is an isomorphism } \\
\text { between } S O(3) \text {-bundles covering } f .
\end{array}
\end{array}\right\} .
$$

This group $\operatorname{Aut}(X, P)$ is the automorphism group of the pair $(X, P)$ in the category of pairs of a 4-manifold and an $S O(3)$-bundle on it. Let $\mathcal{O}$ be a homology orientation of $X$, and define

$$
\operatorname{Aut}(X, P, \mathcal{O}):=\{(f, \tilde{f}) \in \operatorname{Aut}(X, P) \mid f \in \operatorname{Diff}(X, \mathfrak{P}, \mathcal{O})\} .
$$

Let $\mathscr{G}_{P}$ be the gauge group of $P \rightarrow X$. Then we have the following exact sequences

$$
1 \rightarrow \mathscr{G}_{P} \rightarrow \operatorname{Aut}(X, P) \rightarrow \operatorname{Diff}(X, \mathfrak{P}) \rightarrow 1
$$

and

$$
1 \rightarrow \mathscr{G}_{P} \rightarrow \operatorname{Aut}(X, P, \mathcal{O}) \rightarrow \operatorname{Diff}(X, \mathfrak{P}, \mathcal{O}) \rightarrow 1
$$


We next consider Seiberg-Witten theory. To avoid taking care with Riemannian metrics, we use $\operatorname{spin}_{G L}^{c}$ structure as in [20] rather than $\operatorname{spin}^{c}$ structure. Let us review the definition of $\operatorname{spin}_{G L}^{c}$ structure. Fix a double covering $G L_{4}^{+}(\mathbb{R})$ of $G L_{4}^{+}(\mathbb{R})$, where $G L_{4}^{+}(\mathbb{R})$ is the group of invertible real $4 \times 4$-matrices with det $>0$. Set

$$
\operatorname{Spin}_{G L}^{c}(4):=\left(\widetilde{G L}_{4}^{+}(\mathbb{R}) \times U(1)\right) / \pm 1 .
$$

We have the natural map $\operatorname{Spin}_{G L}^{c}(4) \rightarrow G L_{4}^{+}(\mathbb{R})$ as the map $\operatorname{Spin}^{c}(4) \rightarrow S O(4)$. We denote by $\operatorname{Fr}_{G L}(X) \rightarrow X$ the frame bundle whose fiber at $x \in X$ is the set of oriented frames of $T_{x} X$. We define a $\operatorname{spin}_{G L}^{c}$ structure on $X$ as a pair $s=\left(P_{G L}, \psi\right)$ consisting of a $\operatorname{Spin}_{G L}^{c}(4)$-bundle $P_{G L} \rightarrow X$ and an isomorpshism

$$
\psi: P_{G L} \times_{\operatorname{Spin}_{G L}^{c}(4)} G L_{4}^{+}(\mathbb{R}) \rightarrow \operatorname{Fr}_{G L}(X)
$$

between $G L_{4}^{+}(\mathbb{R})$-bundles. Given a $\operatorname{spin}_{G L}^{c}$ structure $s$, a $\operatorname{spin}^{c}$ structure $s_{g}$ is induced corresponding to each Riemannian metric $g$ on $X$. For a fixed metric, an isomorphism class of $\operatorname{spin}_{G L}^{c}$ structures corresponds one-to-one with an isomorphism class of $\operatorname{spin}^{c}$ structures. We do not therefore distinguish $\operatorname{spin}_{G L}^{c}$ structure from $\operatorname{spin}^{c}$ structure when we consider them at the level of isomorphism classes.

Let $s=\left(P_{G L}, \psi\right)$ be a $\operatorname{spin}_{G L}^{c}$ structure on $X, \mathfrak{s}$ be the isomorpshim class of $s$, and $\mathcal{O}$ be a homology orientation of $X$. We have already defined the groups $\operatorname{Diff}(X, \mathfrak{s})$ and $\operatorname{Diff}(X, \mathfrak{s}, \mathcal{O})$ in the introduction. The automorphism group of the pair $(X, s)$ in the category of $\operatorname{spin}_{G L}^{c} 4$-manifolds is given as

$\operatorname{Aut}(X, s):=\left\{\begin{array}{l|c}f \in \operatorname{Diff}(X, \mathfrak{s}), \\ \tilde{f}) \mid \begin{array}{c}\tilde{f}: P_{G L} \rightarrow P_{G L} \text { is an isomorphism between } \\ \operatorname{Spin}_{G L}^{c}(4) \text {-bundles such that the diagram (5) commutes. }\end{array}\end{array}\right\}$,

where (5) is given by

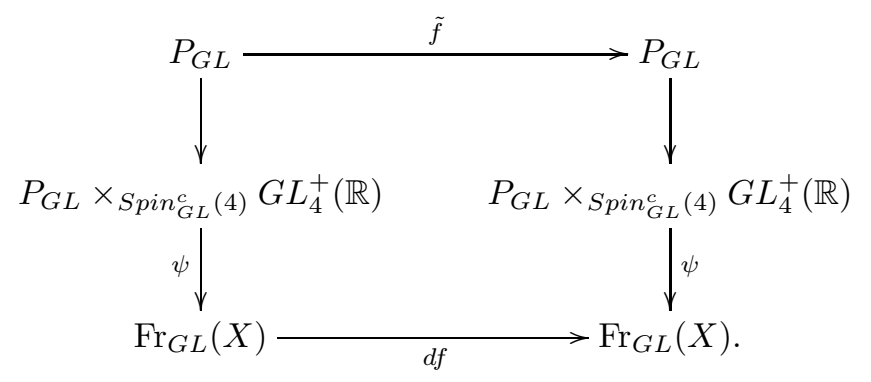

We also define

$$
\operatorname{Aut}(X, s, \mathcal{O}):=\{(f, \tilde{f}) \in \operatorname{Aut}(X, s) \mid f \in \operatorname{Diff}(X, \mathfrak{s}, \mathcal{O})\} .
$$

For the gauge group $\mathscr{G}_{s}$ of the $\operatorname{spin}_{G L}^{c}$ structure $s$, we have exact sequences

$$
1 \rightarrow \mathscr{G}_{s} \rightarrow \operatorname{Aut}(X, s) \rightarrow \operatorname{Diff}(X, \mathfrak{s}) \rightarrow 1
$$

and

$$
1 \rightarrow \mathscr{G}_{s} \rightarrow \operatorname{Aut}(X, s, \mathcal{O}) \rightarrow \operatorname{Diff}(X, \mathfrak{s}, \mathcal{O}) \rightarrow 1 .
$$

For a given metric $g$ on $X$, it is easy to see that the gauge group of $s$ is isomorphic to that of the induced $\operatorname{spin}^{c}$ structure $s_{g}$; both gauge groups are isomorphic to $\operatorname{Map}\left(X, S^{1}\right)$. 


\section{ASD SETting AND SW SETting}

Most of the story of this paper is common to ASD and Seiberg-Witten equations. We present here a summary of settings in which we work for both equations. Let $X$ be an oriented closed smooth 4-manifold and $B$ be a topological space. The first setting is on ASD equations, to which we refer as the $A S D$ setting:

- An $S O(3)$-bundle $P \rightarrow X$ with $w_{2}(P) \neq 0$ is given. Let $\mathfrak{P}$ be the isomorphism class of $P$.

- We write $\mathscr{G}$ for the gauge group $\mathscr{G}_{P}$ of $P$ which is $L_{k+1}^{2}$-completed for $k>1$.

- The groups $G$ and $\tilde{G}$ are either one of the following (11) and (2):

(1) $G=\operatorname{Diff}(X, \mathfrak{P})$ and $\tilde{G}=\operatorname{Aut}(X, P)$.

(2) $G=\operatorname{Diff}(X, \mathfrak{P}, \mathcal{O})$ and $\tilde{G}=\operatorname{Aut}(X, P, \mathcal{O})$ for a given homology orientation $\mathcal{O}$.

- A continuous fiber bundle $X \rightarrow E \rightarrow B$ with structure group $G$ is given.

- For the single 4 -manifold $X$, define $\Pi(X):=\operatorname{Met}(X)$, where $\operatorname{Met}(X)$ is the space of Riemannian metrics on $X$. We also define $\Pi(E):=\bigsqcup_{b \in B} \Pi\left(E_{b}\right)$.

- For each $g \in \Pi(X)$, define

$$
\mathscr{C}_{g}:=\mathscr{A}_{L_{k}^{2}}(P), \quad \mathscr{D}_{g}:=L_{k-1}^{2}\left(\Lambda_{g}^{+}(X) \otimes \operatorname{ad} P\right),
$$

where $\mathscr{A}_{L_{k}^{2}}(P)$ is the space of $S O(3)$-connections on $P$ which is $L_{k}^{2}$-completed, $\Lambda_{g}^{+}(X)$ is the $g$-self-dual part of $\Lambda^{2} T^{*} X$, ad $P \rightarrow X$ is the adjoint bundle associated with $P$ with fiber $\mathfrak{s o}(3)$, and $L_{k-1}^{2}(\cdot)$ means the completion. In fact $\mathscr{C}_{g}$ is independent of $g$ in this case, we use this notation. Let $\mathscr{C}_{g}^{*}$ be the subset of $\mathscr{C}_{g}$ consisting of reducible connections.

- Let $\tilde{s}_{g}: \mathscr{C}_{g} \rightarrow \mathscr{D}_{g}$ be the map defined by the ASD equation

$$
A \mapsto F_{A}^{+g} .
$$

- We call the integer $d$ defined by the formula

$$
d=-2 p_{1}(\mathfrak{P})-3\left(1-b_{1}(X)+b^{+}(X)\right)
$$

the formal dimension of the moduli space of the solutions to the $S O(3)$-ASD equation.

The second setting is on Seiberg-Witten equations, to which we refer as the $S W$ setting:

- A $\operatorname{spin}_{G L}^{c}$ structure $s=\left(P_{G L}, \psi\right)$ is given. Let $\mathfrak{s}$ be the isomorphism class of $s$.

- We write $\mathscr{G}$ for the gauge group $\mathscr{G}_{s}$ of $s$ which is $L_{k+1}^{2}$-completed for $k>1$.

- The groups $G$ and $\tilde{G}$ are either one of the following (1) and (2):

(1) $G=\operatorname{Diff}(X, \mathfrak{s})$ and $\tilde{G}=\operatorname{Aut}(X, s)$.

(2) $G=\operatorname{Diff}(X, \mathfrak{s}, \mathcal{O})$ and $\tilde{G}=\operatorname{Aut}(X, s, \mathcal{O})$ for a given homology orientation $\mathcal{O}$.

- A continuous fiber bundle $X \rightarrow E \rightarrow B$ with structure group $G$ is given.

- For the single 4-manifold $X$, define $\Pi(X):=\bigsqcup_{g \in \operatorname{Met}(X)} L_{k-1}^{2}\left(\Lambda_{g}^{+}(X)\right)$. We also define $\Pi(E):=\bigsqcup_{b \in B} \Pi\left(E_{b}\right)$.

- Let $\pi: \Pi(X) \rightarrow \operatorname{Met}(X)$ be the projection. For each $\mu \in \Pi(X)$, define $\mathscr{C}_{\mu}:=\mathscr{A}_{L_{k}^{2}}(s) \times L_{k}^{2}\left(S_{\pi(\mu)}^{+}\right), \mathscr{D}_{\mu}:=L_{k-1}^{2}\left(i \Lambda_{\pi(\mu)}^{+}(X)\right) \times L_{k-1}^{2}\left(S_{\pi(\mu)}^{-}\right)$ 
where $\mathscr{A}_{L_{k}^{2}}(s)$ is the $L_{k}^{2}$-completion of the space of $U(1)$-connections of the determinant line bundle of $s$, and $S_{\pi(\mu)}^{ \pm}$is the positive and negative spinor bundles of the $\operatorname{spin}^{c}$ structure $s_{\pi(\mu)}$ induced from $s$ corresponding to the metric $\pi(\mu)$. Let $\mathscr{C}_{\mu}^{*}$ be the subset of $\mathscr{C}_{\mu}$ consisting of reducible configurations.

- Let $\tilde{s}_{\mu}: \mathscr{C}_{\mu} \rightarrow \mathscr{D}_{\mu}$ be the map defined by the Seiberg-Witten equations

$$
(A, \Phi) \mapsto\left(F_{A}^{+\pi(\mu)}+i \mu-\rho^{-1}(\sigma(\Phi, \Phi)), D_{A} \Phi\right),
$$

where $\rho: \Lambda_{\pi(\mu)}^{+}(X) \rightarrow \mathfrak{s u}\left(S_{\pi(\mu)}^{+}\right)$is the map obtained from the Clifford multiplication, $\sigma(\cdot, \cdot)$ is the quadratic form given by $\sigma(\Phi, \Phi)=\Phi \otimes \Phi^{*}-$ $|\Phi|^{2} \mathrm{id} / 2$, and $D_{A}: L_{k}^{2}\left(S_{\pi(\mu)}^{+}\right) \rightarrow L_{k-1}^{2}\left(S_{\pi(\mu)}^{-}\right)$is the Dirac operator.

- We call the integer $d$ defined by the formula

$$
d=\left(c_{1}(\mathfrak{s})^{2}-2 \chi(X)-3 \operatorname{sign}(X)\right) / 4
$$

the formal dimension of the moduli space of the solutions to the SeibergWitten equation.

In both settings, we use the following notations

$$
\mathscr{G}, \quad G, \quad \tilde{G}, \quad X \rightarrow E \rightarrow B, \quad \Pi(X), \quad \Pi(E), \quad \tilde{s}_{\bullet}: \mathscr{C}_{\bullet} \rightarrow \mathscr{D}_{\bullet}, \quad \mathscr{C}_{\bullet}^{*}(\bullet \in \Pi(X))
$$

as we defined above. We refer to the case that we consider $G=\operatorname{Diff}(X, \mathfrak{P}, \mathcal{O})$ and $\tilde{G}=\operatorname{Aut}(X, P, \mathcal{O})$, or $G=\operatorname{Diff}(X, \mathfrak{s}, \mathcal{O})$ and $\tilde{G}=\operatorname{Aut}(X, s, \mathcal{O})$ as the homology oriented case. For each $\bullet \in \Pi(X)$, we can define the moduli space of the ASD/Seiberg-Witten equations corresponding to $\bullet$ by

$$
\mathcal{M}_{\bullet}:=\tilde{s}_{\bullet}^{-1}(0) / \mathscr{G} \text {. }
$$

We also define

$$
\mathscr{B}_{\bullet}:=\mathscr{C}_{\bullet} / \mathscr{G}, \quad \mathscr{B}_{\bullet}^{*}:=\mathscr{C}_{\bullet}^{*} / \mathscr{G}, \quad \mathscr{E}_{\bullet}:=\mathscr{C}_{\bullet}^{*} \times \mathscr{G} \mathscr{D}_{\bullet}
$$

Recall that $\mathscr{B}_{\bullet}^{*}$ is a paracompact Hausdorff Hilbert manifold and $\mathscr{E}_{\bullet}$ is a Hilbert bundle on $\mathscr{B}_{\bullet}^{*}$ with fiber $\mathscr{D}_{\bullet}$. Since the map $\tilde{s}_{\bullet}: \mathscr{C}_{\bullet}^{*} \rightarrow \mathscr{D}_{\bullet}$ is $\mathscr{G}_{\text {-equivariant, this }}$ defines a section

$$
s_{\bullet}: \mathscr{B}_{\bullet}^{*} \rightarrow \mathscr{E}_{\bullet}
$$

\section{Cocycle condition modulo gauge}

In Section [6] we shall construct characteristic classes with structure groups $\operatorname{Diff}(X, \mathfrak{P})$, $\operatorname{Diff}(X, \mathfrak{s})$, and the versions of them obtained by adding $\mathcal{O}$ given in Section 2 In the usual theory of characteristic classes, the natural candidate of the structure group is the automorphism group of the fiber in the category in which the fiber belongs. However, our groups are not the automorphism groups in the categories arising from gauge theory, i.e. in the category of the pairs of a 4-manifold and an $S O(3)$-bundle on it and that of $\operatorname{spin}^{c} 4$-manifolds. (This has been noticed in M. Szymik [41.) To resolve it we use N. Nakamura's idea [33] for families of ASD/Seiberg-Witten equations. The idea can be regarded as a "global version" of D. Ruberman's idea given in [38 40] used to define invariants of diffeomorphisms of a 4-manifold. We also note that this kind of idea is initially given by M. Furuta [14] for consideration of group action rather than that of family. Although Nakamura has considered the idea for parameterized moduli spaces, we apply the idea also to families of Fredholm sections themselves arising from ASD/Seiberg-Witten equations. This is because 
we shall use a virtual neighborhood technique in Section 6 6 Let us choose one of the ASD setting or the SW setting, and henceforth work on it in this section.

We first recall the observation due to Ruberman [38 40]: each $f \in G$ defines a well-defined "isomorphism"

$$
f^{*}: \mathcal{M}_{\bullet} \rightarrow \mathcal{M}_{f^{*} \bullet}
$$

between the moduli spaces for any $\bullet \in \Pi(X)$. Here the term "isomorphism" means, for example, a diffeomorphism if $\bullet$ is generic (and the moduli $\mathcal{M}_{\bullet}$ is a manifold). This map is defied using a lift $\tilde{f} \in \tilde{G}$ of $f$ as follows. Recall we have the exact sequence

$$
1 \rightarrow \mathscr{G} \rightarrow \tilde{G} \rightarrow G \rightarrow 1 .
$$

The pull-back by $\tilde{f}$ defines an invertible map

$$
\tilde{f}^{*}: \mathscr{C}_{\bullet} \rightarrow \mathscr{C}_{f^{*} \bullet}
$$

The gauge group $\mathscr{G}$ is a normal subgroup of $\tilde{G}$ because of (7), and therefore the map (8) induces a map between quotients

$$
\tilde{f}^{*}: \mathscr{B}_{\bullet} \rightarrow \mathscr{B}_{f^{*} \bullet}
$$

This map (9) is independent of the choice of lift $\tilde{f}$ again because of the exact sequence (7). We therefore write $f^{*}: \mathscr{B}_{\bullet} \rightarrow \mathscr{B}_{f^{*}} \bullet$ for the map (9), and define the map (6) as the restriction of this map.

We next use Nakamura's idea [33] of considering the "cocycle condition modulo gauge" to construct the parameterized moduli space which is globally defined on the whole of $B$. Fix a section $\sigma: B \rightarrow \Pi(E)$. Since $G$ is not the automorphism group in the suitable categories in both settings, we cannot write down families of ASD/Seiberg-Witten equations corresponding to $\sigma$. Nevertheless, we can consider the moduli space parameterized by $\sigma$ on the whole of $B$ as follows. Let $\left\{U_{\alpha}\right\}_{\alpha}$ be an open covering of $B$ satisfying that $U_{\alpha} \cap U_{\beta}$ is contractible for any $\alpha, \beta$. (We can take such a covering by mimicking the argument, for example given in Hatcher's book [16], to prove that a CW complex is locally contractible.) We write $U_{\alpha \beta}$ and $U_{\alpha \beta \gamma}$ for $U_{\alpha} \cap U_{\beta}$ and $U_{\alpha} \cap U_{\beta} \cap U_{\gamma}$ respectively. Take local trivializations of $E \rightarrow B$ on this covering and let $\left\{g_{\alpha \beta}: U_{\alpha \beta} \rightarrow G\right\}_{\alpha, \beta}$ be the transition functions corresponding to the local trivializations. Since $U_{\alpha \beta}$ is contractible for each $\alpha, \beta$, there exists a lift $\tilde{g}_{\alpha \beta}: U_{\alpha \beta} \rightarrow \tilde{G}$ of $g_{\alpha \beta}$. Note that $\left\{\tilde{g}_{\alpha \beta}\right\}$ satisfies the "cocycle condition modulo gauge", namely,

$$
\tilde{g}_{\alpha \beta} \tilde{g}_{\beta \gamma} \tilde{g}_{\gamma \alpha}(b) \in \mathscr{G}
$$

holds for any $b \in U_{\alpha \beta \gamma}$ since $\left\{g_{\alpha \beta}\right\}$ satisfies the cocycle condition and we have the exact sequence (7). The given section $\sigma: B \rightarrow \Pi(E)$ corresponds to a system of maps $\left\{\sigma_{\alpha}: U_{\alpha} \rightarrow \Pi(X)\right\}_{\alpha}$ satisfying that $\sigma_{\alpha}=g_{\alpha \beta} \cdot \sigma_{\beta}$ on $U_{\alpha \beta}$. Here the action of $g_{\alpha \beta}$ to $\sigma_{\beta}$ is given by the action of $G$ on $\Pi(X)$ via $G \hookrightarrow \operatorname{Diff}^{+}(X)$, namely, $g_{\alpha \beta} \cdot \sigma_{\beta}=g_{\alpha \beta}^{*} \sigma_{\beta}$. For each $\alpha$, let us define the "locally defined" parameterized moduli space

$$
\mathcal{M}_{\sigma_{\alpha}}:=\bigsqcup_{b \in U_{\alpha}} \mathcal{M}_{\sigma_{\alpha}(b)} .
$$

For each point $b \in U_{\alpha \beta}$, we obtain an invertible map

$$
\tilde{g}_{\alpha \beta}(b)^{*}: \mathcal{M}_{\sigma_{\beta}(b)} \rightarrow \mathcal{M}_{g_{\alpha \beta}(b)^{*} \sigma_{\beta}(b)}
$$


like (6). (In fact this map is independent of lift $\tilde{g}_{\alpha \beta}$ as in the definition of (6), we keep using the notation $\tilde{g}_{\alpha \beta}^{*}$.) Because of the relation $\sigma_{\alpha}=g_{\alpha \beta} \cdot \sigma_{\beta}=g_{\alpha \beta}^{*} \sigma_{\beta}$ on $U_{\alpha \beta}$, we eventually have

$$
\tilde{g}_{\alpha \beta}^{*}:\left.\left.\mathcal{M}_{\sigma_{\beta}}\right|_{U_{\alpha \beta}} \rightarrow \mathcal{M}_{\sigma_{\alpha}}\right|_{U_{\alpha \beta}}
$$

The composition

$$
\tilde{g}_{\alpha \beta}^{*} \circ \tilde{g}_{\beta \gamma}^{*} \circ \tilde{g}_{\gamma \alpha}^{*}:\left.\left.\mathcal{M}_{\sigma_{\alpha}}\right|_{U_{\alpha \beta \gamma}} \rightarrow \mathcal{M}_{\sigma_{\alpha}}\right|_{U_{\alpha \beta \gamma}}
$$

coincides with the identity because of (10). This is again a consequence of the definition of the moduli space: it is the quotient space by the gauge group. We can therefore obtain the well-defined quotient space:

Definition 4.1. We define the space $\mathcal{M}_{\sigma}$ equipped with a map $\mathcal{M}_{\sigma} \rightarrow B$ by

$$
\mathcal{M}_{\sigma}:=\bigsqcup_{\alpha} \mathcal{M}_{\sigma_{\alpha}} / \sim
$$

where the equivalence relation $\sim$ is given by the invertible maps $\left\{\tilde{g}_{\alpha \beta}^{*}\right\}$. We refer to the space $\mathcal{M}_{\sigma}$ as the (globally defined) parameterized moduli space.

We now consider this idea at the level of Fredholm sections. Namely, we construct the "globally defined" family of Fredholm sections as follows. For a fixed section $\sigma: B \rightarrow \Pi(E)$, set

$$
\mathscr{B}_{\sigma_{\alpha}}^{*}:=\bigsqcup_{b \in U_{\alpha}} \mathscr{B}_{\sigma_{\alpha}(b)}^{*}, \quad \mathscr{E}_{\sigma_{\alpha}}:=\bigsqcup_{b \in U_{\alpha}} \mathscr{E}_{\sigma_{\alpha}(b)}, \quad s_{\sigma_{\alpha}}:=\bigsqcup_{b \in U_{\alpha}} s_{\sigma_{\alpha}(b)}: \mathscr{B}_{\sigma_{\alpha}}^{*} \rightarrow \mathscr{E}_{\sigma_{\alpha}}
$$

Let us take $f \in G$ and $\bullet \in \Pi(X)$. Then we can consider the invertible map $f^{*}$ : $\mathscr{B}_{\bullet} \rightarrow \mathscr{B}_{f^{*} \bullet}$ as in the definition of ([6). The restriction of this map gives an invertible map $f^{*}: \mathscr{B}_{\bullet}^{*} \rightarrow \mathscr{B}_{f^{*}}^{*}$ between irreducible configurations. We can therefore define a parameterized Hilbert manifold and a parameterized Hilbert bundle using $\mathscr{B}_{\bullet}^{*}$ and $\mathscr{E}_{\bullet}:$

Definition 4.2. We define

$$
\mathscr{B}_{\sigma}^{*}:=\bigsqcup_{\alpha} \mathscr{B}_{\sigma_{\alpha}}^{*} / \sim
$$

by the same argument to define $\mathcal{M}_{\sigma}$ as in Definition 4.1. This is a Hilbert manifold bundle parameterized on $B$. Similarly we can define a parameterized Hilbert bundle $\mathscr{E}_{\sigma} \rightarrow \mathscr{B}_{\sigma}^{*} \rightarrow B$ by

$$
\mathscr{E}_{\sigma}:=\bigsqcup_{\alpha} \mathscr{E}_{\sigma_{\alpha}} / \sim .
$$

In addition, the Fredholm sections themselves are also obtained as the quotient of the gauge group, and we can define:

Definition 4.3. We define a family of Fredholm sections $s_{\sigma}: \mathscr{B}_{\sigma}^{*} \rightarrow \mathscr{E}_{\sigma}$ parameterized on $B$ by

$$
s_{\sigma}:=\bigsqcup_{\alpha} s_{\sigma_{\alpha}} / \sim .
$$




\section{VIRTUAL NEIGHBORHOOD FOR FAMILIES}

One of the precepts of Atiyah-Singer index theory for families 4 is that the natural class of base spaces for a theory on families of Fredholm operators is not smooth manifolds: the smoothness of base spaces is an excessive assumption. Therefore, in our theory of characteristic classes, we do not assume that the base space of a given family of ASD/Seiberg-Witten equations is a smooth manifold. (In fact, we shall eventually extend our theory to families on any topological space.) The problem here is, of course, how to deal with the transversality to count the moduli spaces parameterized on a non-smooth space. To justify this counting argument, we describe a family version of Y. Ruan's virtual neighborhood technique [37. This provides a foundation for our construction of characteristic classes in Section 6 .

5.1. Virtual neighborhood. In this subsection we consider the usual (i.e. unparameterized) virtual neighborhood. The purposes of this subsection is to rewrite the counting argument in the virtual neighborhood technique in terms of the relative Euler class: although Ruan has defined the invariant associated with a given nonlinear Fredholm section of a given Hilbert bundle using the finite dimensional Sard's theorem, we define the invariant using the relative Euler class given in M. Kervaire 17] instead of Sard's theorem. We note that P. Feehan and T. Leness 11, 12 have used Ruan's technique in a similar form. This description of the invariant allows us easily to extend Ruan's technique to families on non-smooth spaces.

We work in the following general setting.

- Let $\mathscr{X}$ be a Hilbert manifold. We assume that $\mathscr{X}$ is paracompact Hausdorff and the model of $\mathscr{X}$ is a separable Hilbert space. These topological conditions are used to assure that there exists a smooth partition of unity on $\mathscr{X}$. (See, for example, Lang [23, Chapter II, Corollary 3.8.)

- Let $\mathscr{H}$ be a Hilbert space and $\mathscr{E} \rightarrow \mathscr{X}$ be a Hilbert bundle with fiber $\mathscr{H}$.

- Let $s: \mathscr{X} \rightarrow \mathscr{E}$ be a Fredholm section.

Here the definition of a Fredholm section in this paper is as follows. For $x \in$ $\mathscr{X}$, we have the canonical decomposition $T_{(x, 0)} \mathscr{E}=T_{x} \mathscr{X} \oplus \mathscr{H}$. We also write $d s_{x}: T_{x} \mathscr{X} \rightarrow \mathscr{H}$ for the composition of $d s_{x}: T_{x} \mathscr{X} \rightarrow T_{(x, 0)} \mathscr{E}$ and the projection $T_{(x, 0)} \mathscr{E}=T_{x} \mathscr{X} \oplus \mathscr{H} \rightarrow \mathscr{H}$. We call a smooth section $s: \mathscr{X} \rightarrow \mathscr{E}$ a Fredholm section if the differential $d s_{x}: T_{x} \mathscr{X} \rightarrow \mathscr{H}$ is a Fredholm map for each $x \in s^{-1}(0)$. We assume that the Fredholm index of $d s_{x}$ is common to all $x \in s^{-1}(0)$ and write ind $s$ for the index. For the zero set

$$
\mathcal{M}:=s^{-1}(0),
$$

we assume the compactness:

Assumption 5.1. The zero set $\mathcal{M}$ is compact.

A typical example of $s$ satisfying this assumption is given as the Seiberg-Witten equations modulo the gauge group. Under this assumption, we shall define the "counted number $\# \mathcal{M}$ " $\in \mathbb{Z}$ or $\mathbb{Z} / 2$, corresponding to the Seiberg-Witten invariant in the case of this example, via a virtual neighborhood.

We first review the construction of a virtual neighborhood. Let $x$ be a point in $\mathcal{M}$. Then there exists $N_{x} \in \mathbb{N}$ and a linear map $f_{x}: \mathbb{R}^{N_{x}} \rightarrow \mathscr{H}$ such that

$$
d s_{x}+f_{x}: T_{x} \mathscr{X} \oplus \mathbb{R}^{N_{x}} \rightarrow \mathscr{H}
$$


is surjective. Since surjectivity are open conditions, there exists a small open neighborhood $U_{x}$ of $x$ in $\mathscr{X}$ such that $d s_{y}+f_{x}: T_{y} \mathscr{X} \oplus \mathbb{R}^{N_{x}} \rightarrow \mathscr{H}$ is surjective for any $y \in U_{x} \cap \mathcal{M}$. Because $\mathcal{M}$ is compact, there exists finite points $x_{1}, \ldots, x_{m} \in \mathcal{M}$ such that $\mathcal{M} \subset \bigcup_{i=1}^{m} U_{x_{i}}$. Set $N_{i}:=N_{x_{i}}, f_{i}:=f_{x_{i}}, U_{i}:=U_{x_{i}}$ and $N:=N_{1}+\cdots+N_{m}$. As we mentioned, we can take a smooth partition of unity $\left\{\rho_{i}\right\}_{i=1}^{m}$ subordinate to $\left\{U_{i}\right\}_{i=1}^{m}$. We also use the notation $\mathscr{E}$ for its pull-back to $\mathscr{X} \times \mathbb{R}^{N}$. Fix a local trivialization of $\mathscr{E}$ on each $U_{i}$, and define a map

$$
\varphi: \mathscr{X} \times \mathbb{R}^{N} \rightarrow \mathscr{E}
$$

by

$$
\left(x,\left(a_{1}, \ldots, a_{m}\right)\right) \mapsto \sum_{i=1}^{m} \rho_{i}(x) f_{i}\left(a_{i}\right),
$$

where $x \in \mathscr{X}$ and $\left(a_{1}, \ldots, a_{m}\right) \in \mathbb{R}^{N_{1}} \times \cdots \times \mathbb{R}^{N_{m}}=\mathbb{R}^{N}$, and the map $\left(x, a_{i}\right) \mapsto$ $\rho_{i}(x) f_{i}\left(a_{i}\right)$ is regarded as a local section of $\mathscr{E}$ via the fixed local trivialization. Note that $\mathscr{X} \times\{0\} \subset \varphi^{-1}(0)$ holds. We call $\varphi$ a finite dimensional perturbation of $s$. For the new section

$$
\tilde{s}:=s+\varphi: \mathscr{X} \times \mathbb{R}^{N} \rightarrow \mathscr{E}
$$

the following lemma is straightforward:

Lemma 5.2 (Ruan [37, Lemma 2.3). For any $x \in \mathcal{M}$, the differential

$$
d \tilde{s}_{(x, 0)}: T_{x} \mathscr{X} \oplus \mathbb{R}^{N} \rightarrow \mathscr{H}
$$

is surjective.

Since surjectivity is an open condition, there exists a neighborhood $\mathscr{N}$ of $\mathcal{M} \times\{0\}$ in $\mathscr{X} \times \mathbb{R}^{N}$ such that the differential of $\tilde{s}$ is surjective on any point of $\left(\left.\tilde{s}\right|_{\mathscr{N}}\right)^{-1}(0)=$ $\tilde{s}^{-1}(0) \cap \mathscr{N}$. Set

$$
\mathcal{U}=\mathcal{U}(s, \varphi):=\tilde{s}^{-1}(0) \cap \mathscr{N} .
$$

Because of the implicit function theorem, $\mathcal{U}$ is a smooth manifold. Since $\operatorname{dim} \operatorname{Ker}\left(d \tilde{s}_{(x, 0)}\right)=$ ind $s+N$ holds for any $x \in \mathscr{X}$, we have

$$
\operatorname{dim} \mathcal{U}=\operatorname{ind} s+N .
$$

Henceforth we regard the zero set $\mathcal{M}$ as a subspace of $\mathcal{U}$ by

$$
\mathcal{M} \cong \mathcal{M} \times\{0\}=\tilde{s}^{-1}(0) \cap(\mathscr{X} \times\{0\}) \cap \mathscr{N} \subset \mathcal{U} .
$$

Definition 5.3 (Ruan [37]). The finite dimensional manifold $\mathcal{U}$ constructed above is called a virtual neighborhood for the Fredholm section $s: \mathscr{X} \rightarrow \mathscr{E}$.

The restriction of the projection $\mathscr{X} \times \mathbb{R}^{N} \rightarrow \mathbb{R}^{N}$ is equipped with for a virtual neighborhood $\mathcal{U}$. We denote by $h_{\mathcal{U}}: \mathcal{U} \rightarrow \mathbb{R}^{N}$ this map. The space $\mathcal{M}$ is the level set of $h_{\mathcal{U}}$ for the height zero. We are interested only in the "germ" of $h_{\mathcal{U}}$ near $\mathcal{M}$, and we do not distinguish $\mathcal{U}$ and another virtual neighborhood $\mathcal{U}^{\prime}$ obtained from the common finite dimensional perturbation $\varphi$ (even if another $\mathscr{N}^{\prime}$ is used instead of $\mathscr{N})$.

Here we remark the orientation of $\mathcal{U}$. The determinant line bundle det $s \rightarrow s^{-1}(0)$ is associated with the section $s: \mathscr{X} \rightarrow \mathscr{H}$. Since $\tilde{s}: \mathscr{X} \times \mathbb{R}^{N} \rightarrow \mathscr{H}$ is also a Fredholm section, we can also define $\operatorname{det} \tilde{s} \rightarrow \tilde{s}^{-1}(0)$. 
Lemma 5.4 (Ruan [37, Lemma 2.4). If $\operatorname{det} s \rightarrow s^{-1}(0)$ has a nowhere-vanishing section, it induces a a nowhere-vanishing section on $\operatorname{det} \tilde{s} \rightarrow \tilde{s}^{-1}(0)$. (Thus a nowhere-vanishing section on $\operatorname{det} s \rightarrow s^{-1}(0)$ gives an orientation of $\mathcal{U}$.)

We now define the "counted number $\# \mathcal{M}$ " in terms of the relative Euler class. Assume that $k:=$ ind $s$ is non-negative and fix a cohomology class $\alpha \in H^{k}(\mathscr{X} ; \mathbb{Z})$. (If we work with $\mathbb{Z} / 2$-coefficient, we can take a general element of $H^{k}(\mathscr{X} ; \mathbb{Z} / 2)$ as $\alpha$.) Then we obtain the cohomology class $p^{*} \alpha \in H^{k}(\mathcal{U} ; \mathbb{Z})$, where $p: \mathcal{U} \rightarrow \mathscr{X}$ is the restriction of the projection $\mathscr{X} \times \mathbb{R}^{N} \rightarrow \mathscr{X}$. The map $h_{\mathcal{U}}: \mathcal{U} \rightarrow \mathbb{R}^{N}$ can be regarded as a section

$$
h_{\mathcal{U}}: \mathcal{U} \rightarrow \mathcal{U} \times \mathbb{R}^{N}
$$

of the trivial bundle $\mathcal{U} \times \mathbb{R}^{N} \rightarrow \mathcal{U}$. (The trivial bundle $\mathcal{U} \times \mathbb{R}^{N} \rightarrow \mathcal{U}$ and the section $h_{\mathcal{U}}: \mathcal{U} \rightarrow \mathcal{U} \times \mathbb{R}^{N}$ can be regarded as a "finite dimensional approximation" of the Hilbert bundle $\mathscr{E} \rightarrow \mathscr{X}$ and one of the Fredholm section $s: \mathscr{X} \rightarrow \mathscr{E}$ respectively.) Since $h_{\mathcal{U}}^{-1}(0) \cong \mathcal{M}$ is compact, one can take a relatively compact neighborhood $K$ of $h_{\mathcal{U}}^{-1}(0)$ in $\mathcal{U}$. Let

$$
\tau\left(\mathcal{U} \times \mathbb{R}^{N}\right) \in H^{N}\left(D\left(\mathcal{U} \times \mathbb{R}^{N}\right), S\left(\mathcal{U} \times \mathbb{R}^{N}\right) ; \mathbb{Z}\right)
$$

be the Thom class of the trivial bundle $\mathcal{U} \times \mathbb{R}^{N} \rightarrow \mathcal{U}$, where $D(\cdot)$ and $S(\cdot)$ mean the disk bundle and sphere bundle respectively. Then one can consider the relative Euler class with respect to $h_{\mathcal{U}}$ :

$$
e_{\mathcal{U}}:=h_{\mathcal{U}}^{*} \tau\left(\mathcal{U} \times \mathbb{R}^{N}\right) \in H^{N}(\mathcal{U}, \mathcal{U} \backslash K ; \mathbb{Z}) .
$$

We use the same notation $e_{\mathcal{U}}$ for the image of $e_{\mathcal{U}}$ by the map

$$
H^{N}(\mathcal{U}, \mathcal{U} \backslash K ; \mathbb{Z}) \rightarrow H_{\mathrm{cpt}}^{N}(\mathcal{U} ; \mathbb{Z}),
$$

where $H_{\mathrm{cpt}}^{*}(\cdot ; \mathbb{Z})$ means the cohomology with compact supports. Since $\mathcal{U}$ is an (open) manifold, we can consider the fundamental class $[\mathcal{U}]_{B M}$ in the sense of BorelMoore homology. Here $[\mathcal{U}]_{B M}$ is regarded as a homology class with $\mathbb{Z}$-coefficient if a nowhere-vanishing section of $\operatorname{det} s$ is given, and otherwise with $\mathbb{Z} / 2$-coefficient.

Definition 5.5. We define the number $\mathfrak{m}(s, \alpha) \in \mathbb{Z}$ or $\mathbb{Z} / 2$ by

$$
\mathfrak{m}(s, \alpha):=\left\langle e_{\mathcal{U}} \cup p^{*} \alpha,[\mathcal{U}]_{B M}\right\rangle .
$$

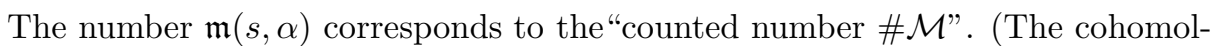
ogy class $\alpha$ corresponds to a cutting of the moduli space of higher dimension.) The proof of the following lemma can be regarded as a non-linearization of the proof of the well-definedness of the index for families.

Remark 5.6. Assume that the Poincaré dual (in the sense of Borel-Moore) of $p^{*} \alpha$ can be represented by a smooth submanifold $V \subset \mathcal{U}$ of $\mathcal{U}$, and also that $\mathcal{M} \cap V=\emptyset$. Then we have $\mathfrak{m}(s, \alpha)=0$.

Lemma 5.7 (corresponding to Ruan [37, Proposition 2.6). The number $\mathfrak{m}(s, \alpha)$ depends only on the section $s: \mathscr{X} \rightarrow \mathscr{H}$ and the cohomology class $\alpha \in H^{k}(\mathscr{X} ; \mathbb{Z})$.

Proof. Let $\mathcal{U}_{i}(i=1,2)$ be two virtual neighborhoods obtained from $\tilde{s}_{i}:=s+\varphi_{i}$ : $\mathscr{X} \times \mathbb{R}^{N_{i}} \rightarrow \mathscr{E}$. Let us consider two maps

$$
\begin{aligned}
& \tilde{s}_{1}^{\prime}:=s+\varphi_{1}+0: \mathscr{X} \times \mathbb{R}^{N_{1}} \times \mathbb{R}^{N_{2}} \rightarrow \mathscr{E}, \text { and } \\
& \tilde{s}_{12}:=s+\varphi_{1}+\varphi_{2}: \mathscr{X} \times \mathbb{R}^{N_{1}} \times \mathbb{R}^{N_{2}} \rightarrow \mathscr{E} .
\end{aligned}
$$


The differential of the map

$$
s+\varphi_{1}+\bullet \varphi_{2}: \mathscr{X} \times \mathbb{R}^{N_{1}} \times \mathbb{R}^{N_{2}} \times[0,1] \rightarrow \mathscr{E}
$$

defined as

$$
\left(x, a_{1}, a_{2}, \tau\right) \mapsto s(x)+\varphi_{1}\left(x, a_{1}\right)+\tau \varphi_{2}\left(x, a_{2}\right)
$$

is surjective at each point on $\mathcal{M} \times\{0\} \times\{0\} \times[0,1]$, and so on a neighborhood of $\mathcal{M} \times\{0\} \times\{0\} \times[0,1]$ in $\mathscr{X} \times \mathbb{R}^{N_{1}} \times \mathbb{R}^{N_{2}} \times[0,1]$. Thus we obtain a manifold $\mathcal{U} \subset \mathscr{X} \times \mathbb{R}^{N_{1}} \times \mathbb{R}^{N_{2}} \times[0,1]$ with boundary from the Fredholm section (16) via the same procedure used to define a virtual neighborhood as above. By replacing $\mathcal{U}$ and virtual neighborhoods to some smaller neighborhoods of $\mathcal{M}$ if we need, $\mathcal{U}$ gives a cobordism between a virtual neighborhood $\mathcal{U}_{1}^{\prime}$ obtained from $\tilde{s}_{1}^{\prime}$ and a virtual neighborhood $\mathcal{U}_{12}$ obtained from $\tilde{s}_{12}$. (Such a manifold $\mathcal{U}$ is called a virtual neighborhood cobordism in Ruan [37.) Let us consider the section

$$
h_{\mathcal{U}}: \mathcal{U} \times[0,1] \rightarrow \mathcal{U} \times \mathbb{R}^{N_{1}} \times \mathbb{R}^{N_{2}} \times[0,1]
$$

and the relative Euler class $e_{\mathcal{U}} \in H_{\mathrm{cpt}}^{N_{1}+N_{2}}(\mathcal{U} \times[0,1] ; \mathbb{Z})$ obtained in a similar way to define (12) and (13). Since the restrictions of $h_{\mathcal{U}}$ to $\tau=0,1$ are the sections $h_{\mathcal{U}_{1}^{\prime}}$ and $h_{\mathcal{U}_{12}}$ respectively, the relative Euler classes $e_{\mathcal{U}_{1}^{\prime}}$ and $e_{\mathcal{U}_{12}}$ are the restrictions of $e_{\mathcal{U}}$ to $\mathcal{U}_{1}^{\prime}$ and to $\mathcal{U}_{12}$ respectively. Thus we have

$$
\left\langle e_{\mathcal{U}_{1}^{\prime}} \cup p^{*} \alpha,\left[\mathcal{U}_{1}^{\prime}\right]_{B M}\right\rangle-\left\langle e_{\mathcal{U}_{12}} \cup p^{*} \alpha,\left[\mathcal{U}_{12}\right]_{B M}\right\rangle=\left\langle e_{\mathcal{U}} \cup p^{*} \alpha, \partial[\mathcal{U}]_{B M}\right\rangle=0,
$$

where $p$ in the left-hand side is the map given by the projection $\mathscr{X} \times \mathbb{R}^{N_{1}} \times \mathbb{R}^{N_{2}} \rightarrow \mathscr{X}$ and $p$ in the right-hand side is that given by $\mathscr{X} \times \mathbb{R}^{N_{1}} \times \mathbb{R}^{N_{2}} \times[0,1] \rightarrow \mathscr{X}$. Let $e_{\mathcal{U}_{1} \times \mathbb{R}^{N_{2}}} \in H_{\mathrm{cpt}}^{N_{1}+N_{2}}\left(\mathcal{U}_{1} \times \mathbb{R}^{N_{2}} ; \mathbb{Z}\right)$ be the relative Euler class defined by the section

$$
h_{\mathcal{U}_{1}} \times \operatorname{id}_{\mathbb{R}^{N_{2}}}: \mathcal{U}_{1} \times \mathbb{R}^{N_{2}} \rightarrow \mathcal{U}_{1} \times \mathbb{R}^{N_{1}} \times \mathbb{R}^{N_{2}} .
$$

Since the virtual neighborhood $\mathcal{U}_{1}^{\prime}$ is same to $\mathcal{U}_{1} \times \mathbb{R}^{N_{2}}$ if we focus only on neighborhoods of $\mathcal{M}$, we have

$$
\left\langle e_{\mathcal{U}_{1}^{\prime}} \cup p^{*} \alpha,\left[\mathcal{U}_{1}^{\prime}\right]_{B M}\right\rangle=\left\langle e_{\mathcal{U}_{1} \times \mathbb{R}^{N_{2}}} \cup p^{*} \alpha,\left[\mathcal{U}_{1} \times \mathbb{R}^{N_{2}}\right]_{B M}\right\rangle .
$$

In addition, since $e_{\mathcal{U}_{1} \times \mathbb{R}^{N_{2}}}$ corresponds to $e_{\mathcal{U}_{1}}$ via the suspension isomorphism

$$
H_{\mathrm{cpt}}^{*}\left(\mathcal{U}_{1} \times \mathbb{R}^{N_{2}} ; \mathbb{Z}\right) \cong H_{\mathrm{cpt}}^{*-N_{2}}\left(\mathcal{U}_{1} ; \mathbb{Z}\right)
$$

we obtain

$$
\left\langle e_{\mathcal{U}_{1} \times \mathbb{R}^{N_{2}}} \cup p^{*} \alpha,\left[\mathcal{U}_{1} \times \mathbb{R}^{N_{2}}\right]_{B M}\right\rangle=\left\langle e_{\mathcal{U}_{1}} \cup p^{*} \alpha,\left[\mathcal{U}_{1}\right]_{B M}\right\rangle .
$$

The equalities (17), (18), and (19) imply that

$$
\left\langle e_{\mathcal{U}_{1}} \cup p^{*} \alpha,\left[\mathcal{U}_{1}\right]_{B M}\right\rangle=\left\langle e_{\mathcal{U}_{12}} \cup p^{*} \alpha,\left[\mathcal{U}_{12}\right]_{B M}\right\rangle .
$$

Via $\left\langle e_{\mathcal{U}_{12}} \cup p^{*} \alpha,\left[\mathcal{U}_{12}\right]_{B M}\right\rangle$, it follows that

$$
\left\langle e_{\mathcal{U}_{1}} \cup p^{*} \alpha,\left[\mathcal{U}_{1}\right]_{B M}\right\rangle=\left\langle e_{\mathcal{U}_{2}} \cup p^{*} \alpha,\left[\mathcal{U}_{2}\right]_{B M}\right\rangle
$$

from the equality (20) and the similar equality relating $\mathcal{U}_{2}$ and $\mathcal{U}_{12}$ shown by the same argument. 
5.2. Family virtual neighborhood. We give a family version of Ruan's virtual neighborhood technique in this subsection. We work in the following setting in this subsection.

- Let $B$ be a normal space. (Then we can take continuous cut-off functions.)

- Let $\pi: \mathscr{X}=\bigsqcup_{b \in B} \mathscr{X}_{b} \rightarrow B$ be a parametrized Hilbert manifold, namely, be a continuous fiber bundle and suppose that each fiber $\mathscr{X}_{b}$ be a paracompact Hausdorff Hilbert manifold whose model Hilbert space is separable.

- Let $\mathscr{E}=\bigsqcup_{b \in B} \mathscr{E}_{b} \rightarrow \bigsqcup_{b \in B} \mathscr{X}_{b} \rightarrow B$ be a parametrized Hilbert bundle. Namely, let $\mathscr{E} \rightarrow B$ be a continuous fiber bundle and suppose that $\mathscr{E}_{b} \rightarrow \mathscr{X}_{b}$ is a smooth Hilbert bundle whose fiber is a Hilbert space $\mathscr{H}_{b}$ for each $b \in B$.

- Let $s=\bigsqcup_{b \in B} s_{b}: \mathscr{X} \rightarrow \mathscr{E}$ be a parametrized Fredholm section, namely, be a continuous section and suppose that the restriction to each fiber $s_{b}$ : $\mathscr{X}_{b} \rightarrow \mathscr{E}_{b}$ is a smooth Fredholm section.

- We assume that the index of $d\left(s_{b}\right)_{x}: T_{x} \mathscr{X}_{b} \rightarrow \mathscr{H}_{b}$ is common to all $b \in B$ and $x \in \mathscr{X}_{b}$ and write ind $s$ for the index. We also assume that $d\left(s_{b}\right)$ continuously depends on $b$. Namely, for the bundles $T_{\text {fiber }} \mathscr{X}=\bigsqcup_{b \in B} T \mathscr{X}_{b}$ and $T_{\text {fiber }} \mathscr{E}=\bigsqcup_{b \in B} T \mathscr{E}_{b}$, the section of $\operatorname{Hom}\left(T_{\text {fiber }} \mathscr{X}, s^{*} T_{\text {fiber }} \mathscr{E}\right) \rightarrow B$ induced from $d s$ is continuous.

Set

$$
\mathcal{M}=\bigsqcup_{b \in B} \mathcal{M}_{b}:=s^{-1}(0)=\bigsqcup_{b \in B} s_{b}^{-1}(0),
$$

corresponding to the parametrized moduli space. We assume that $s$ satisfies the following assumption on compactness:

Assumption 5.8. The space $\mathcal{M}$ is compact.

We now construct a "family virtual neighborhood". Let $x$ be a point in $\mathcal{M}$. Then there exists $N_{x} \in \mathbb{N}$ and a linear map $f_{x}$ such that

$$
d\left(s_{\pi(x)}\right)_{x}+f_{x}: T_{x} \mathscr{X}_{\pi(x)} \oplus \mathbb{R}^{N_{x}} \rightarrow \mathscr{H}_{\pi(x)}
$$

is surjective. Take a small neighborhood $U_{x}$ of $x$ in $\mathscr{X}$ such that $d\left(s_{\pi(y)}\right)_{y}+f_{x}$ is surjective for any $y \in U_{x} \cap \mathcal{M}$. We can choose $U_{x}$ such as it is the product of open sets $U_{x}^{\text {base }}$ of $B$ and $U_{x}^{\text {fiber }}$ of the fiber $\mathscr{X}_{b}$ via a fixed local trivialization of $\mathscr{X} \rightarrow B$, i.e. $U_{x} \cong U_{x}^{\text {base }} \times U_{x}^{\text {fiber }}$. Let $p_{x}: U_{x}^{\text {base }} \times U_{x}^{\text {fiber }} \rightarrow U_{x}^{\text {fiber }}$ be the projection. Take a (continuous) cut-off function $\rho_{x}^{\text {base }}: U_{x}^{\text {base }} \rightarrow[0,1]$ supported in $U_{x}^{\text {base }}$ satisfying $\rho_{x}^{\text {base }}(x)>0$, and do a smooth partition of unity $\left\{\rho_{x}^{\text {fiber }}\right\}_{x \in \mathcal{M}_{b}}$ subordinate to $\left\{U_{x}^{\text {fiber }}\right\}_{x \in \mathcal{M}_{b}}$ in $\mathscr{X}_{b}$. Define

$$
\rho_{x}: U_{x} \rightarrow[0,1]
$$

as

$$
\rho_{x}^{\text {base }} \cdot p_{x}^{*} \rho_{x}^{\text {fiber }}: U_{x}^{\text {base }} \times U_{x}^{\text {fiber }} \rightarrow[0,1]
$$

via the fixed local trivialization. Take an open set $U_{x}^{\prime}$ in $\mathscr{X}$ with $x \in U_{x}^{\prime} \subset U_{x}$ such that $\rho_{x}>0$ on $U_{x}^{\prime}$. Then $\left\{U_{x}^{\prime}\right\}_{x \in \mathcal{M}}$ is an open covering of $\mathcal{M}$, and hence there exists finite points $x_{1}, \ldots, x_{m} \in \mathcal{M}$ such that $\mathcal{M} \subset \bigcup_{i=1}^{m} U_{x_{i}}$. Set $N_{i}:=N_{x_{i}}$, $f_{i}:=f_{x_{i}}, U_{i}:=U_{x_{i}}$, and $N:=N_{1}+\cdots+N_{m}$. Fix a local trivialization of $\mathscr{E}$ on each $U_{i}$, and define a map

$$
\varphi: \mathscr{X} \times \mathbb{R}^{N} \rightarrow \mathscr{E}
$$


by the formula (11) via the fixed local trivializations. Set

$$
\begin{aligned}
& \tilde{s}:=s+\varphi: \mathscr{X} \times \mathbb{R}^{N} \rightarrow \mathscr{E}, \text { and } \\
& \tilde{s}_{b}:=s_{b}+\varphi: \mathscr{X}_{b} \times \mathbb{R}^{N} \rightarrow \mathscr{E}_{b} .
\end{aligned}
$$

We now have the following lemma by the completely same argument to prove Lemma 5.2 since $\rho_{i}$ is smooth along the fiber direction of $\mathscr{X} \rightarrow B$ and for any $x \in \mathcal{M}$ there exists $i$ such that $\rho_{i}(x)>0$ :

Lemma 5.9. For any $b \in B$ and any $x \in \mathcal{M}_{b}$, the differential

$$
d\left(\tilde{s}_{b}\right)_{(x, 0)}: T_{x} \mathscr{X}_{b} \oplus \mathbb{R}^{N} \rightarrow \mathscr{H}_{b}
$$

is surjective.

From this lemma, there exists a neighborhood $\mathscr{N}$ of $\mathcal{M} \times\{0\}$ in $\mathscr{X} \times \mathbb{R}^{N}$ such that such that the differential of $\tilde{s}_{b}$ is surjective on any point of $\left(\left.\tilde{s}_{b}\right|_{\mathscr{N}}\right)^{-1}(0)=$ $\tilde{s}_{b}^{-1}(0) \cap \mathscr{N}$ for any $b$. Set

$$
\begin{aligned}
& \mathcal{U}=\mathcal{U}(s, \varphi):=\tilde{s}^{-1}(0) \cap \mathscr{N}, \text { and } \\
& \mathcal{U}_{b}:=\tilde{s}_{b}^{-1}(0) \cap \mathscr{N} .
\end{aligned}
$$

Then each $\mathcal{U}_{b}$ is a smooth manifold with $\operatorname{dim} \mathcal{U}_{b}=\operatorname{ind} s+N$.

Definition 5.10. The family of manifolds

$$
\mathcal{U}=\mathcal{U}(s, \varphi)=\bigsqcup_{b \in B} \mathcal{U}_{b}
$$

constructed above is called a family virtual neighborhood for the parametrized Fredholm section $s: \mathscr{X} \rightarrow \mathscr{H}$.

As in the non-parameterized case, the restriction of the projection $\mathscr{X} \times \mathbb{R}^{N} \rightarrow \mathbb{R}^{N}$ is equipped with for a family virtual neighborhood $\mathcal{U}$. We denote by $h_{\mathcal{U}}: \mathcal{U} \rightarrow \mathbb{R}^{N}$ this map. The space $\mathcal{M}$ is regarded as a subspace of $\mathcal{U}$ and is the level set of $h_{\mathcal{U}}$ for the height zero.

Remark 5.11. We note that a family virtual neighborhood $\mathcal{U}$ is not a fiber bundle in general, and also note that, although $\mathcal{U}$ is parameterized on the whole of $B$, it is "supported" on the subspace

$$
B^{s}:=\left\{b \in B \mid s_{b}^{-1}(0) \neq \emptyset\right\},
$$

i.e. if $s_{b}^{-1}(0)=\emptyset$ holds, then a small neighborhood of $\mathcal{M}_{b}$ in $\mathcal{U}_{b}$ is also empty.

We now construct a (compactly supported) cohomology class on $B$ from a family virtual neighborhood. For $k \geq 0$, fix a cohomology class $\alpha$ in $H^{k}(\mathscr{X} ; \mathbb{Z})$ or $H^{k}(\mathscr{X} ; \mathbb{Z} / 2)$. Let $p: \mathcal{U} \rightarrow \mathscr{X}$ be the restriction of the projection $\mathscr{X} \times \mathbb{R}^{N} \rightarrow \mathscr{X}$. The map $h_{\mathcal{U}}: \mathcal{U} \rightarrow \mathbb{R}^{N}$ can be regarded as a section

$$
h_{\mathcal{U}}: \mathcal{U} \rightarrow \mathcal{U} \times \mathbb{R}^{N}
$$

of the trivial bundle $\mathcal{U} \times \mathbb{R}^{N} \rightarrow \mathcal{U}$. Since $h_{\mathcal{U}}^{-1}(0) \cong \mathcal{M}$ is compact, one can take a relatively compact neighborhood $K$ of $h_{\mathcal{U}}^{-1}(0)$ in $\mathcal{U}$. Let us consider the relative Euler class with respect to $h_{\mathcal{U}}$ :

$$
e_{\mathcal{U}}:=h_{\mathcal{U}}^{*} \tau\left(\mathcal{U} \times \mathbb{R}^{N}\right) \in H^{N}(\mathcal{U}, \mathcal{U} \backslash K ; \mathbb{Z})
$$


using the Thom class $\tau\left(\mathcal{U} \times \mathbb{R}^{N}\right)$ of the trivial bundle $\mathcal{U} \times \mathbb{R}^{N} \rightarrow \mathcal{U}$. We use the same notation $e_{\mathcal{U}}$ for the image of $e_{\mathcal{U}}$ by the map

$$
H^{N}(\mathcal{U}, \mathcal{U} \backslash K ; \mathbb{Z}) \rightarrow H_{\mathrm{cpt}}^{N}(\mathcal{U} ; \mathbb{Z}) .
$$

Since $\mathcal{M}$ is compact and $\mathcal{U}$ is supported on $B^{s}$, one can find a fiberwise embedding $\mathcal{U} \rightarrow B \times \mathbb{R}^{n}$ for large $n$. Although $\mathcal{U} \rightarrow B$ is not a fiber bundle in general, one can therefore define "integration along the fiber"

$$
\pi_{!}: H_{\mathrm{cpt}}^{*}(\mathcal{U}) \rightarrow H_{\mathrm{cpt}}^{*-(\text { ind } s+N)}(B)
$$

as follows. For each $b \in B$, let $\nu_{\mathcal{U}_{b}} \rightarrow \mathcal{U}_{b}$ be the normal bundle for the embedding $\mathcal{U}_{b} \hookrightarrow\{b\} \times \mathbb{R}^{n}$, and let $\nu_{\mathcal{U}} \rightarrow \mathcal{U}$ be the "parametrized normal bundle", i.e. $\nu_{\mathcal{U}}:=$ $\bigsqcup_{b \in B} \nu_{\mathcal{U}_{b}} \rightarrow \mathcal{U} \rightarrow B$. Then $\nu_{\mathcal{U}} \rightarrow \mathcal{U}$ is a continuous vector bundle on $\mathcal{U}$ of rank $n-($ ind $s+N)$. We regard $\nu_{\mathcal{U}}$ as a small neighborhood of $\mathcal{U}$ in $B \times \mathbb{R}^{n}$. Using the Thom isomorphism and the excision isomorphism, we get the map

$$
\begin{aligned}
H^{*}(\mathcal{U}) & \cong H^{*+n-(\text { ind } s+N)}(D(\nu \mathcal{U}), S(\nu \mathcal{U})) \\
& \cong H^{*+n-(\text { ind } s+N)}\left(D\left(B \times \mathbb{R}^{n}\right), \overline{D\left(B \times \mathbb{R}^{n}\right) \backslash \nu \mathcal{U}}\right) \\
& \rightarrow H^{*+n-(\text { ind } s+N)}\left(D\left(B \times \mathbb{R}^{n}\right), S\left(B \times \mathbb{R}^{n}\right)\right) \cong H^{*-(\text { ind } s+N)}(B) .
\end{aligned}
$$

This map induces a map between compact supported cohmology grourps, and we define (21) as the map. Here the coefficient of the cohomology groups in (21) is $\mathbb{Z}$ if a nowhere-vanishing section of $\operatorname{det} s \rightarrow s^{-1}(0)$ is given, and otherwise $\mathbb{Z} / 2$. The map (21) is independent of the choice of fiberwise embedding as usual.

Definition 5.12. We define a cohomology class $\mathfrak{M}(s, \alpha)$ by the formula

$$
\mathfrak{M}(s, \alpha):=\pi_{!}\left(e_{\mathcal{U}} \cup p^{*} \alpha\right) \in H_{\mathrm{cpt}}^{k-\text { ind } s}(B) .
$$

Let $B^{\prime} \subset B$ be a subset satisfying $B^{\prime} \cap B^{s}=\emptyset$, i.e. $s$ is nowhere-vanishing on $B^{\prime}$. Then the cohomology class (22) can be regarded as an element of the relative cohomology:

$$
\mathfrak{M}\left(s, \alpha ; B^{\prime}\right):=\pi_{!}\left(e_{\mathcal{U}} \cup p^{*} \alpha\right) \in H_{\mathrm{cpt}}^{k-i n d} s\left(B, B^{\prime}\right) .
$$

Here the coefficient of the cohomology groups in (22) and (23) is $\mathbb{Z}$ if a nowherevanishing section of $\operatorname{det} s \rightarrow s^{-1}(0)$ is given, and otherwise is $\mathbb{Z} / 2$. We call $\mathfrak{M}(s, \alpha)$ and $\mathfrak{M}\left(s, \alpha ; B^{\prime}\right)$ the cohomological invariants emerging from $s, \alpha$ (and $B^{\prime}$ for the latter case).

Remark 5.13. Assume that the fiberwise Poincaré dual of $p^{*} \alpha$ can be represented as a family of smooth submanifolds $V \subset \mathcal{U}, V=\bigsqcup_{b \in B} V_{b}$. For a subspace $B^{\prime} \subset B$, suppose that $\mathcal{M}_{b} \cap V_{b}=\emptyset$ for any $b \in B^{\prime}$. Then, as in Remark 5.6, the cohomology class $\mathfrak{M}(s, \alpha)$ can be regarded as an element of the relative cohomology group; we can define $\mathfrak{M}\left(s, \alpha ; B^{\prime}\right) \in H_{\mathrm{cpt}}^{k-\text { ind } s}\left(B, B^{\prime}\right)$ as in (23).

Remark 5.14. For a given family of 4-manifolds, assume that the family of ASD or Seiberg-Witten equations arising from the family of 4-manifolds satisfies the condition corresponding to Assumption 5.8. Then we can obtain a cohomology class on the base space by combing Definition 5.12 with the argument given in Section 2, However, for a general family on a non-compact base (for example the universal bundle of the diffeomorphism group on the classifying space of it), there is no standard way to assure the condition corresponding to Assumption 5.8 To establish the universal theory, we need an argument obtained by mimicking the obstruction theory, given in Section 6 . 
Lemma 5.15. The cohomology class $\mathfrak{M}(s, \alpha)$ depends only on the section $s: \mathscr{X} \rightarrow$ $\mathscr{E}$ and the cohomology class $\alpha \in H^{k}(\mathscr{X} ; \mathbb{Z})$. Similarly, if $s: \mathscr{X} \rightarrow \mathscr{E}$ is nowherevanishing on a subspace $B^{\prime} \subset B$, the cohomology class $\mathfrak{M}\left(s, \alpha ; B^{\prime}\right)$ depends only on $s: \mathscr{X} \rightarrow \mathscr{E}, \alpha \in H^{k}(\mathscr{X} ; \mathbb{Z})$, and $B^{\prime}$.

Proof. Let $\mathcal{U}_{i}(i=1,2)$ be two virtual neighborhoods obtained from $\tilde{s}_{i}:=s+\varphi_{i}$ : $\mathscr{X} \times \mathbb{R}^{N_{i}} \rightarrow \mathscr{E}$ and consider two maps $\tilde{s}_{1}^{\prime}$ and $\tilde{s}_{12}$ defined by the same formulae (14) and (15). For each $b \in B$, the differential of

$$
s_{b}+\varphi_{1}+\bullet \varphi_{2}: \mathscr{X}_{b} \times \mathbb{R}^{N_{1}} \times \mathbb{R}^{N_{2}} \times[0,1] \rightarrow \mathscr{E}_{b} \times[0,1]
$$

is surjective at each point on $\mathcal{M}_{b} \times\{0\} \times\{0\} \times[0,1]$, and thus on a neighborhood of $\mathcal{M}_{b} \times\{0\} \times\{0\} \times[0,1]$ in $\mathscr{X}_{b} \times \mathbb{R}^{N_{1}} \times \mathbb{R}^{N_{2}} \times[0,1]$. Thus we obtain a family of manifolds with boundaries $\mathcal{U} \subset \mathscr{X} \times \mathbb{R}^{N_{1}} \times \mathbb{R}^{N_{2}} \times[0,1]$ and we can assume that $\mathcal{U}$ gives a fiberwise cobordism between a family virtual neighborhood $\mathcal{U}_{1}^{\prime}$ obtained from $\tilde{s}_{1}^{\prime}$ and a family virtual neighborhood $\mathcal{U}_{12}$ obtained from $\tilde{s}_{12}$. The relative Euler classes $e_{\mathcal{U}_{1}^{\prime}}$ and $e_{\mathcal{U}_{12}}$ are the restrictions of the relative Euler class obtained from $h_{\mathcal{U}}$ to $\mathcal{U}_{1}^{\prime}$ and $\mathcal{U}_{12}$ respectively. Therefore, using the following elementary Lemma 5.16 we have $\pi_{!}\left(e_{\mathcal{U}_{1}^{\prime}} \cup p^{*} \alpha\right)=\pi_{!}\left(e_{\mathcal{U}_{12}} \cup p^{*} \alpha\right)$. The rest of the proof is an argument based on the suspension isomorphism, which is same as the proof of Lemma 5.7.

Lemma 5.16. Let $\mathcal{V}_{0} \rightarrow B, \mathcal{V}_{1} \rightarrow B$ and $\mathcal{V} \rightarrow B$ be continuous maps (not necessary fiber bundles). Assume that the inverse images by these maps of each point of $B$ are smooth manifolds, and also assume that they admit fiberwise embeddings into a trivial vector bundle. (Then one can define integration along the fiber for these families.) Suppose that $\mathcal{V}$ gives a fiberwise cobordism between $\mathcal{V}_{0}$ and $\mathcal{V}_{1}$. Then, for any cohomology class $\beta \in H_{\mathrm{cpt}}^{*}(\mathcal{V})$,

$$
\pi_{!}\left(\left.\beta\right|_{\mathcal{E}_{0}}\right)=\pi_{!}\left(\left.\beta\right|_{\mathcal{E}_{1}}\right)
$$

holds.

Proof. Since integration along the fiber commutes with restriction, it is enough to show our statement in the case that $\mathcal{V}_{i}$ and $\mathcal{V}$ are trivial bundles. In this case, one can easily to see it using the Künneth formula and the cobordism argument as (17).

Definition 5.17. (1) If $B$ is a closed manifold of dimension of $k$ - ind $s$, we define a number $\mathfrak{m}(s, \alpha)$ by

$$
\mathfrak{m}(s, \alpha):=\langle\mathfrak{M}(s, \alpha),[B]\rangle \in \mathbb{Z} \text { or } \mathbb{Z} / 2 .
$$

Here, if a nowhere-vanishing section of $\operatorname{det} s \rightarrow s^{-1}(0)$ is given and if $B$ is oriented, $\mathfrak{m}(s, \alpha) \in \mathbb{Z}$, and otherwise $\in \mathbb{Z} / 2$.

(2) If $B$ is a compact manifold with boundary and $s$ is nowhere-vanishing on $\partial B$, we define a number $\mathfrak{m}(s, \alpha ; \partial B)$ by

$$
\mathfrak{m}(s, \alpha ; \partial B):=\langle\mathfrak{M}(s, \alpha ; \partial B),[B, \partial B]\rangle \in \mathbb{Z} \text { or } \mathbb{Z} / 2 .
$$

Here whether $\mathfrak{m}(s, \alpha ; \partial B)$ is in $\mathbb{Z}$ or not is similar to the case (1).

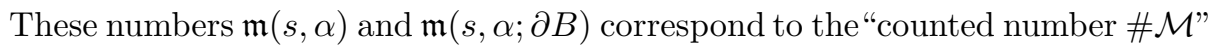
for the parameterized moduli space on $B$.

We remark a lemma relating the "naturality" at the end of this section: 
Lemma 5.18. Let $\mathscr{E} \rightarrow \mathscr{X} \rightarrow B$ and $s: \mathscr{X} \rightarrow \mathscr{E}$ be as above, $A$ be a normal space, and $f: A \rightarrow B$ be a continuous map. Assume that, for the pull-backed section $f^{*} s: f^{*} \mathscr{X} \rightarrow f^{*} \mathscr{E}$, the parameterized zero set $\left(f^{*} s\right)^{-1}(0)$ is also compact. Let $\mathcal{U}$ be a family virtual neighborhood for the parameterized Fredholm section $s$. Then, there exist a family virtual neighborhood $f^{*} \mathcal{U}$ for the pull-backed section $f^{*} s$ and a continuous map $\tilde{f}: f^{*} \mathcal{U} \rightarrow \mathcal{U}$ such that $\tilde{f}$ covers $f$ and

$$
\tilde{f}^{*} e_{\mathcal{U}}=e_{f * \mathcal{U}}
$$

holds.

Proof. Let $\varphi: \mathscr{X} \times \mathbb{R}^{N} \rightarrow \mathscr{E}$ be the finite dimensional perturbation used to define $\mathcal{U}$. Then $f^{*} \mathcal{U}$ is constructed as the virtual neighborhood obtained from $f^{*} \varphi$ : $f^{*} \mathscr{X} \times \mathbb{R}^{N} \rightarrow f^{*} \mathscr{E}$. Let $\bar{f}: f^{*} \mathscr{X} \rightarrow \mathscr{X}$ be the natural map covering $f$. Then the map $\tilde{f}$ is given as the restriction of $\bar{f} \times \operatorname{id}_{\mathbb{R}^{N}}: f^{*} \mathscr{X} \times \mathbb{R}^{N} \rightarrow \mathscr{X} \times \mathbb{R}^{N}$. If we take the open manifold $\mathscr{N}$ used in the definition of $\mathcal{U}$ and that for $f^{*} \mathcal{U}$ to be sufficiently small, one can check that the equality

$$
\left(\tilde{f} \times \operatorname{id}_{\mathbb{R}^{N}}\right)^{*} \tau\left(\mathcal{U} \times \mathbb{R}^{N}\right)=\tau\left(f^{*} \mathcal{U} \times \mathbb{R}^{N}\right)
$$

between the Thom classes holds in

$$
H^{N}\left(f^{*} \mathcal{U} \times D\left(\mathbb{R}^{N}\right), f^{*} \mathcal{U} \times S\left(\mathbb{R}^{N}\right)\right) \cong H^{0}\left(f^{*} \mathcal{U}\right) \otimes R,
$$

where $R=\mathbb{Z}$ or $\mathbb{Z} / 2$ is the coefficient ring. This implies the equality (24) between the Euler classes.

Corollary 5.19. Let $\mathscr{E} \rightarrow \mathscr{X} \rightarrow B, s: \mathscr{X} \rightarrow \mathscr{E}$, and $f: A \rightarrow B$ be that given in Lemma 5.18 and assume that $\left(f^{*} s\right)^{-1}(0)$ is also compact. Then

$$
f^{*} \mathfrak{M}(s, \alpha)=\mathfrak{M}\left(s, \bar{f}^{*} \alpha\right)
$$

holds for any $\alpha \in H^{*}(\mathscr{X})$, where $\bar{f}: f^{*} \mathscr{X} \rightarrow \mathscr{X}$ is the natural map covering $f$. Similarly, for subsets $A^{\prime} \subset A$ and $B^{\prime} \subset B$ satisfying that $f\left(A^{\prime}\right) \subset B^{\prime}$ and that $s$ is nowhere-vanishing on $A^{\prime}$, we have

$$
f^{*} \mathfrak{M}\left(s, \alpha ; B^{\prime}\right)=\mathfrak{M}\left(s, \bar{f}^{*} \alpha ; A^{\prime}\right) .
$$

Proof. This is because pull-back commutes with integration along the fiber.

\section{Construction of the Characteristic Classes}

The aim of this section is to construct characteristic classes bundles of 4-manifolds via ASD/Seiberg-Witten equations. The procedure of the construction is some analogy of that of obstruction theory. The basic reason why the analogy works is that we have an interpretation of the Donaldson/Seiberg-Witten invariants as the "Euler classes" of some Hilbert bundles, explained as the introduction. To consider the Euler class in the rigorous sense, we use some finite dimensional approximations of the Hilbert bundles, given in Section 5. We note that the story of this section is similar to that of Section 3 in [19, in which the author has given the construction of the cohomological Seiberg-Witten invariant associated with the adjunction complex of surfaces. 
6.1. Main construction. Let $X$ be an oriented closed smooth 4-manifold. Let us choose one of the ASD setting or the SW setting, and work on it in this subsection. We shall eventually define characteristic classes for bundles on a general topological space, but let $B$ be a CW complex until Corollary 6.7. Let $n$ be a non-negative integer and suppose that $b^{+}(X) \geq n+2$. Assume that the formal dimension of the moduli space is $-n$. In this subsection we define our characteristic classes under these assumptions. Let $B^{(n)}$ denote the $n$-skeleton of $B$. We first construct a section

$$
\sigma=\sigma^{(n)}:\left.B^{(n)} \rightarrow \Pi(E)\right|_{B^{(n)}}
$$

inductively as follows. For each $b \in B^{(0)}$, take a generic point in $\Pi\left(E_{b}\right)$. Then we have $\sigma^{(0)}:\left.B^{(0)} \rightarrow \Pi(E)\right|_{B^{(0)}}$. Assume that we have constructed $\sigma^{(k-1)}$ : $\left.B^{(k-1)} \rightarrow \Pi(E)\right|_{B^{(k-1)}}$ for $k \leq n$ such that the parameterized moduli space for $\sigma^{(k-1)}$ is empty: $\mathcal{M}_{\sigma^{(k-1)}}=\emptyset$. Here $\mathcal{M}_{\sigma^{(k-1)}}$ is the space obtained by substituting $\sigma^{(k-1)}$ for $\sigma$ in Definition 4.1. Note that this condition on $\sigma^{(k-1)}$ implies that $\mathcal{M}_{\sigma^{(k-1)}}$ contains no reducible solution. Let $e \subset B$ be a $k$-cell and $\varphi_{e}: D_{e}^{k} \rightarrow$ $\bar{e} \subset B$ be the characteristic map of $e$. Here $D_{e}^{k}$ is the standard $k$-dimensional disk indexed by $e$. Since the bundle $\varphi_{e}^{*} \Pi(E) \rightarrow D_{e}^{k}$ is trivial, we can take a trivialization $\psi_{e}: \varphi_{e}^{*} \Pi(E) \rightarrow D_{e}^{k} \times \Pi(X)$. Let us consider the continuous map obtained as the composition

$$
p_{2} \circ \psi_{e} \circ\left(\left.\varphi_{e}\right|_{\partial D_{e}^{k}}\right)^{*} \sigma^{(k-1)}: \partial D_{e}^{k} \rightarrow\left(\left.\varphi_{e}\right|_{\partial D_{e}^{k}}\right)^{*} \Pi(E) \rightarrow \partial D_{e}^{k} \times \Pi(X) \rightarrow \Pi(X),
$$

where $p_{2}: \partial D_{e}^{k} \times \Pi(X) \rightarrow \Pi(X)$ is the projection. Since $b^{+}(X) \geq n+1$, we can smoothly and generically extend this map to a map from $D_{e}^{k}$ into $\Pi(X)$ avoiding the wall. (Here the term "smoothly" means smoothness in the interior of $D_{e}^{k}$.) This extended map gives a section $\left.\bar{e} \rightarrow \Pi(E)\right|_{\bar{e}}$. We therefore obtain $\sigma^{(k)}: B^{(k)} \rightarrow$ $\left.\Pi(E)\right|_{B^{(k)}}$. In fact this procedure can be continued until $k=n+1$ since we assume $b^{+}(X) \geq n+2$, but we stop it until $k=n$ in this subsection. (The case that $k=n+1$ is used in Subsection 6.2) We call such $\sigma$ an inductive section. Note that whether $\mathcal{M}_{\sigma^{(k)}}$ is empty or not is independent of the choice of local trivialization. This is because another choice of trivialization induces a bijection as in (6). To detect $\mathcal{M}_{\sigma^{(k)}}$ is empty or not, we can therefore use the smooth structure of the pull-backed moduli space on $D_{e}^{n-1}$ via the trivializations $\psi_{e}$. Thus we have $\mathcal{M}_{\sigma^{(k)}}=\emptyset$ for $k<n$ because of formal dimension.

For each $n$-cell $e$, substituting $\varphi_{e}^{*} \sigma$ for $\sigma_{\sigma}$ in Definition 4.3, we can consider the family of Fredholm sections $s_{\varphi_{e}^{*} \sigma}$ corresponding to $\varphi_{e}^{*} \sigma$ and parameterized on $D_{e}^{n}$. As we mentioned, the section (25) satisfies that $\mathcal{M}_{\sigma^{(n-1)}}=\emptyset$. Namely, for each $n$-cell $e$, the family of Fredholm sections $s_{\varphi_{e}^{*} \sigma}$ is nowhere-vanishing on $\partial D_{e}^{n}$. In addition, the parameterized moduli space $\mathcal{M}_{\varphi_{e}^{*} s}$ is compact because of the compactness of $D_{e}^{n}$ and that of the usual (i.e. unparameterized) moduli space of the solutions to the $S O(3)$-ASD equation with non-trivial $w_{2}$ or to the Seiberg-Witten equations. In other words, the family of Fredholm sections $s_{\varphi_{e}^{*} \sigma}$ satisfies the compactness assumption corresponding to Assumption 5.8. We can therefore have the number

$$
\mathfrak{m}\left(s_{\varphi_{e}^{*} \sigma}, 1 ; D_{e}^{n}\right) \in \mathbb{Z} \text { or } \mathbb{Z} / 2
$$

by substituting $s_{\varphi_{e}^{*} \sigma}$ and $1 \in H^{0}\left(\mathscr{B}_{\varphi_{e}^{*} \sigma}^{*}\right)$ for $s$ and $\alpha$ in Definition 5.17, where $\mathscr{B}_{\varphi_{e}^{*} \sigma}^{*}$ is the Hilbert manifold obtained by substituting $\varphi_{e}^{*} \sigma$ for $\sigma$ in Definition 4.2 Here if 
we consider the homology oriented case, the number is in $\mathbb{Z}$, and otherwise in $\mathbb{Z} / 2$. We write

$$
C_{*}(B), \quad C^{*}(B), \quad \partial: C_{*}(B) \rightarrow C_{*-1}(B), \quad \delta: C^{*}(B) \rightarrow C^{*+1}(B)
$$

for the (cellular) chain complex, the cochain complex, the boundary operator, and the coboundary operator respectively, where the coefficient is $\mathbb{Z}$ if we consider the homology oriented case, and otherwise is $\mathbb{Z} / 2$. In what follows, we keep this convention on coefficient.

Definition 6.1. For the section $\sigma$ constructed above, we define a cochain

$$
\mathcal{A}(E, \sigma) \in C^{n}(B)
$$

by

$$
e \mapsto \mathfrak{m}\left(s_{\varphi_{e}^{*} \sigma}, 1 ; D_{e}^{n}\right) .
$$

We shall prove the following proposition in Subsection 6.2

Proposition 6.2. The cochain $\mathcal{A}(E, \sigma)$ constructed above is a cocycle.

We now may write down the definition of our characteristic classes:

Definition 6.3. We define

$$
\mathbb{A}(E):=[\mathcal{A}(E, \sigma)] \in H^{n}(B),
$$

where $\sigma:\left.B^{(n)} \rightarrow \Pi(E)\right|_{B^{(n)}}$ is an inductive section.

This cohomology class is invariants of $E$ :

Theorem 6.4. The cohomology class $\mathbb{A}(E)$ given in Definition 6.3 is independent of the choice of $\sigma$.

We shall prove Theorem 6.4 in Subsection 6.2 .

Remark 6.5. If we assume Theorem 6.4 we can immediately see that, if $E$ is a trivial $G$-bundle and $n>0$, then $\mathbb{A}(E)=0$. Indeed, in this case, we can take a "constant" inductive section $\sigma$, then we have $\mathcal{A}(E, \sigma)=0$ because of formal dimension.

In addition, $\mathbb{A}(\cdot)$ satisfies functoriality. Namely, $\mathbb{A}(\cdot)$ is a characteristic class:

Theorem 6.6. The correspondence $E \mapsto \mathbb{A}(E)$ is functorial. Namely, for a $C W$ complex $B^{\prime}$ and a continuous map $f: B^{\prime} \rightarrow B$, we have

$$
f^{*} \mathbb{A}(E)=\mathbb{A}\left(f^{*} E\right) \text {. }
$$

We shall prove Theorem 6.6 in Subsection 6.2 By applying Theorem 6.6 to the identity map, we also have the following independence of $\mathbb{A}(E)$ on $\mathrm{CW}$ structure of the base space:

Corollary 6.7. The cohomology class $\mathbb{A}(E)$ given in Definition 6.3 is independent of the choice of $C W$ structure of $B$.

In fact, we can functorially extend the definition of $\mathbb{A}(\cdot)$ to any bundle on any topological space using a purely topological lemma:

Lemma 6.8. For any topological space $B$, we can associate a cohomology class $\mathbb{A}(E) \in H^{n}(B)$ to any continuous fiber bundle $X \rightarrow E \rightarrow B$ with structure group $G$, and this correspondence $E \mapsto \mathbb{A}(E)$ satisfies that: 
- if $B$ is a $C W$ complex, this $\mathbb{A}(E)$ coincides with that given in Definition 6.3, and

- for a topological space $B^{\prime}$ and a continuous map $f: B^{\prime} \rightarrow B$, we have $f^{*} \mathbb{A}(E)=\mathbb{A}\left(f^{*} E\right)$.

Proof. Recall that, for any topological space $B$, there exists a CW complex $|\Delta(B)|$ equipped with a weak homotopy equivalence map $\rho_{B}:|\Delta(B)| \rightarrow B$. The space $|\Delta(B)|$ is obtained by considering the geometric realization of the simplicial set arising from singular simplices, and this construction $B \mapsto|\Delta(B)|$ is functorial with respect to the map $\rho_{B}:|\Delta(B)| \rightarrow B$. (For example, see May's book [26].) For a bundle $X \rightarrow E \rightarrow B$, let us define

$$
\mathbb{A}(E):=\left(\rho_{B}^{*}\right)^{-1} \mathbb{A}\left(\rho_{B}^{*} E\right) \in H^{n}(B),
$$

where $\mathbb{A}(\cdot)$ in the right-hand side is the one defined in Definition 6.3, Then it is straightforward to check the required conditions.

Write $\mathbb{D}(\cdot):=\mathbb{A}(\cdot)$ and $\mathbb{S W}(\cdot):=\mathbb{A}(\cdot)$ for the ASD setting and the SW setting respectively. We summarize the results in this subsection as follows.

Theorem 6.9. Let $n$ be a non-negative integer, $X$ be an oriented closed smooth 4-manifold with $b^{+}(X) \geq n+2$, and $B$ be a topological space.

(1) Let $\mathfrak{P}$ be the isomorphism class of an $S O(3)$-bundle satisfying that $w_{2}(\mathfrak{P}) \neq$ 0 and $d(\mathfrak{P})=-n$.

(a) To a continuous fiber bundle $X \rightarrow E \rightarrow B$ with structure group $\operatorname{Diff}(X, \mathfrak{P})$, we can associate

$$
\mathbb{D}(E) \in H^{n}(B ; \mathbb{Z} / 2) .
$$

(b) To a continuous fiber bundle $X \rightarrow E \rightarrow B$ with structure group $\operatorname{Diff}(X, \mathfrak{P}, \mathcal{O})$, we can associate

$$
\mathbb{D}(E) \in H^{n}(B ; \mathbb{Z}) .
$$

In both case of (1a) and (1b), the correspondence $E \mapsto \mathbb{D}(E)$ is functorial.

(2) Let $\mathfrak{s}$ be the isomorphism class of a $\operatorname{spin}^{c}$ structure on $X$ with $d(\mathfrak{s})=-n$.

(a) To a continuous fiber bundle $X \rightarrow E \rightarrow B$ with structure group $\operatorname{Diff}(X, \mathfrak{s})$, we can associate

$$
\mathbb{S W}(E) \in H^{n}(B ; \mathbb{Z} / 2) .
$$

(b) To a continuous fiber bundle $X \rightarrow E \rightarrow B$ with structure group $\operatorname{Diff}(X, \mathfrak{s}, \mathcal{O})$, we can associate

$$
\mathbb{S W}(E) \in H^{n}(B ; \mathbb{Z}) .
$$

In both case of (2a) and (2b), the correspondence $E \mapsto \mathbb{S W}(E)$ is functorial.

Definition 6.10. In the setting of Theorem 6.9, we define

$$
\begin{aligned}
& \mathbb{D}(X, \mathfrak{P}):=\mathbb{D}(E \operatorname{Diff}(X, \mathfrak{P})) \in H^{n}(B \operatorname{Diff}(X, \mathfrak{P}) ; \mathbb{Z} / 2), \\
& \mathbb{D}(X, \mathfrak{P}, \mathcal{O}):=\mathbb{D}(E \operatorname{Diff}(X, \mathfrak{P}, \mathcal{O})) \in H^{n}(B \operatorname{Diff}(X, \mathfrak{P}, \mathcal{O}) ; \mathbb{Z}) \\
& \mathbb{S W}(X, \mathfrak{s}):=\mathbb{S W}(E \operatorname{Diff}(X, \mathfrak{s})) \in H^{n}(B \operatorname{Diff}(X, \mathfrak{s}) ; \mathbb{Z} / 2), \text { and } \\
& \mathbb{S W}(X, \mathfrak{s}, \mathcal{O}):=\mathbb{S W}(E \operatorname{Diff}(X, \mathfrak{s}, \mathcal{O})) \in H^{n}(B \operatorname{Diff}(X, \mathfrak{s}, \mathcal{O}) ; \mathbb{Z})
\end{aligned}
$$


Of course the statement of Theorem 6.9 has no meaning if we cannot show the non-triviality of them. In Section 8 we shall explicitly calculate some of these characteristic classes. Note that, in the case that $n=0$, the cohomology classes given in Definition 6.10 are nothing other than the usual $S O(3)$-Donaldson invariants and Seiberg-Witten invariants (valued in $\mathbb{Z} / 2$ and in $\mathbb{Z}$ ) defined by counting the moduli space of formal dimension zero. Therefore for $n=0$ theses classes are obviously non-trivial, and so we are interested in the non-triviality in the case that $n>0$, which is the subject of Section 8 .

Remark 6.11. We have assumed that the formal dimension of the moduli space is $-n$ in this subsection. We can relax the assumptions using non-trivial cohomology class $\alpha$ appeared in Section 5, which corresponds to cutting of higher-dimensional moduli spaces. However, the following technical issue arises to consider such a generalization of characteristic classes. Let us focus on the SW case for simplicity. In the unparameterized situation, to obtain a cohomology class of positive degree on the irreducible configuration space divided by the gauge group, denoted by $\mathscr{B}^{*}$, one needs to consider an $S^{1}$-fibration $S^{1} \rightarrow \mathcal{L} \rightarrow \mathscr{B}^{*}$. The total space $\mathcal{L}$ is given as the quotient of the configuration space divided by a subgroup $\mathscr{G}_{0}$ of $\mathscr{G}$ inducing an exact sequence

$$
1 \rightarrow \mathscr{G}_{0} \rightarrow \mathscr{G} \rightarrow S^{1} \rightarrow 1 .
$$

Because of the rest symmetry of $S^{1}$, we cannot apply the argument of Section 4 to $\mathcal{L}$ : for a family with structure group $\operatorname{Diff}(X, \mathfrak{s})$ or $\operatorname{Diff}(X, \mathfrak{s}, \mathcal{O})$, we cannot obtain a line bundle which is globally defined on the whole base space by gluing together $\mathcal{L}$ 's. However, we hope one can avoid this problem by considering some stacks rather than line bundles, which will be discussed in a subsequent paper.

6.2. Well-definedness and naturality. The purpose of this subsection is to prove Proposition 6.2 and Theorems 6.4 and 6.6. Before starting it, we note the following lemma on an expression of the cochain $\mathcal{A}(\cdot)$. Henceforth we identify $C^{n}(B)$ with $H^{n}\left(B^{(n)}, B^{(n-1)}\right)$.

Lemma 6.12. In the setting of Definition 6.1, assume that $B$ is compact. Then we have

$$
\mathfrak{M}\left(s_{\sigma}, 1 ; B^{(n-1)}\right)=\mathcal{A}(E, \sigma)
$$

in $H^{n}\left(B^{(n)}, B^{(n-1)}\right)=C^{n}(B)$.

Proof. We first note that the left-hand side of (26) can be defined in $H^{n}\left(B^{(n)}, B^{(n-1)}\right)$ since $B$ is compact and $\mathcal{M}_{\sigma^{(n-1)}}=\emptyset$ holds. Let $e$ be an $n$-cell of $B$ and $\varphi_{e}: D_{e}^{n} \rightarrow B$ be its characteristic map. Because of the compactness of $B$ and $D_{e}^{n}$, we can apply Corollary 5.19 to $\varphi_{e}: D_{e}^{n} \rightarrow B$, and thus we obtain

$$
\varphi_{e}^{*} \mathfrak{M}\left(s_{\sigma}, 1 ; B^{(n-1)}\right)=\mathfrak{M}\left(\varphi_{e}^{*} s_{\sigma}, 1 ; \partial D_{e}^{n}\right)
$$

The equality and the isomorphism

$$
\prod_{e \subset B} \varphi_{e}^{*}: C^{n}(B)=H^{n}\left(B^{(n)}, B^{(n-1)}\right) \rightarrow \prod_{e \subset B} H^{n}\left(D_{e}^{n}, \partial D_{e}^{n}\right)
$$

imply (26).

We now prove the naturality at the level of cochains. 
Proposition 6.13. Let us follow the setting of Definition 6.1. Then, for a $C W$ complex $B^{\prime}$ and a cellular map $f: B^{\prime} \rightarrow B$,

$$
f^{*} \mathcal{A}(E, \sigma)=\mathcal{A}\left(f^{*} E, f^{*} \sigma\right)
$$

holds in $C^{n}\left(B^{\prime}\right)$.

Proof. We first note that the notation $\mathcal{A}\left(f^{*} E, f^{*} \sigma\right)$ makes sense: $f^{*} \sigma$ is also an inductive section on $B^{\prime(n)}$, which is a straightforward verification. Let $e \subset B^{\prime}$ be an $n$-cell and $\varphi_{e}: D_{e}^{n} \rightarrow B^{\prime}$ be the characteristic map of $e$. Take a finite subcomplex $K^{\prime} \subset B^{\prime}$ containing the image of $\varphi_{e}$, and also take a finite subcomplex $K \subset B$ containing the image $f\left(K^{\prime}\right)$. By applying Lemma 6.12 to $\left.\sigma\right|_{K^{(n)}}$, we have

$$
\mathfrak{M}\left(s_{\left.\sigma\right|_{K}(n)}, 1 ; K^{(n-1)}\right)=i_{K}^{*} \mathcal{A}(E, \sigma)
$$

in $C^{n}\left(K^{(n)}\right)=C^{n}(K)$, where $i_{K}: K \hookrightarrow B$ is the inclusion. On the other hand, because of the compactness of $K^{(n)}, K^{\prime(n)}$ and $D_{e}^{n}$, we can apply Corollary 5.19 to the restriction $f: K^{\prime(n)} \rightarrow K^{(n)}$ of $f$ and $\varphi_{e}$, and thus have

$$
\varphi_{e}^{*} f^{*} \mathfrak{M}\left(s_{\left.\sigma\right|_{K}(n)}, 1 ; K^{(n-1)}\right)=\mathfrak{M}\left(\varphi_{e}^{*} f^{*} s_{\left.\sigma\right|_{K}(n)}, 1 ; \partial D_{e}^{n}\right) .
$$

Since $\varphi_{e}^{*} f^{*} s_{\sigma}=s_{\varphi_{e}^{*} f^{*} \sigma}$ holds, the equalities (28) and (29) imply that

$$
\varphi_{e}^{*} f^{*} \mathcal{A}(E, \sigma)=\mathfrak{M}\left(s_{\varphi_{e}^{*} f^{*} \sigma}, 1 ; \partial D_{e}^{n}\right) .
$$

It therefore follows that

$$
\begin{aligned}
& f^{*} \mathcal{A}(E, \sigma)(e)=\left\langle f^{*} \mathcal{A}(E, \sigma), e\right\rangle=\left\langle\varphi_{e}^{*} f^{*} \mathcal{A}(E, \sigma),\left[D_{e}^{n}, \partial D_{e}^{n}\right]\right\rangle \\
= & \left\langle\mathfrak{M}\left(s_{\varphi_{e}^{*} f^{*} \sigma}, 1 ; \partial D_{e}^{n}\right),\left[D_{e}^{n}, \partial D_{e}^{n}\right]\right\rangle=\left\langle\mathcal{A}\left(f^{*} E, f^{*} \sigma\right), e\right\rangle .
\end{aligned}
$$

Thus we obtain (27).

Proof of Theorem 6.6. This is a direct consequence of Proposition 6.13 and the cellular approximation theorem. (Here we interpret $\mathbb{A}\left(f^{*} E\right)$ as $\left[\mathcal{A}\left(f^{*} E, f^{*} \sigma\right)\right]$, which is in fact independent of $f^{*} \sigma$ by Proposition 6.2.)

We now give the proof of Proposition 6.2 and Theorem 6.4. Both of them are shown using $(n+1)$-parameter families, corresponding to so-called arguments by cobordisms. However, in fact, any cobordism between manifolds does not explicitly appear in this subsection. This is because it has been absorbed into the well-definedness of the cohomological invariants (Lemma 5.15) in family virtual neighborhood context.

Proof of Proposition 6.2. As we noted in Subsection 6.1 an inductive section $\sigma^{(\bullet)}$ can be constructed also for $\bullet=n+1$ since $b^{+}(X) \geq n+2$. Let us fix $\sigma^{(n+1)}$ : $\left.B^{(n+1)} \rightarrow \Pi(E)\right|_{B^{(n+1)}}$ through the inductive procedure in Subsection 6.1. By abuse of notation, we write $\sigma$ for both $\sigma^{(n)}$ and $\sigma^{(n+1)}$. Take an $(n+1)$-cell $e \subset B$ and its characteristic map $\varphi_{e}: \Delta^{n+1} \rightarrow B$. Here we equip $D_{e}^{n} \cong \Delta^{n+1}$ with the $\mathrm{CW}$ structure as the standard simplex, and by cellular approximation, we can assume that $\varphi_{e}$ is cellular with respect to this CW structure on $\Delta^{n+1}$. From Proposition 6.13 it follows that

$$
\begin{aligned}
& \delta \mathcal{A}(E, \sigma)(e)=\delta \mathcal{A}(E, \sigma)\left(\varphi_{e *} \Delta^{n+1}\right)=\varphi_{e}^{*} \delta \mathcal{A}(E, \sigma)\left(\Delta^{n+1}\right) \\
= & \delta \varphi_{e}^{*} \mathcal{A}(E, \sigma)\left(\Delta^{n+1}\right)=\delta \mathcal{A}\left(\varphi_{e}^{*} E, \varphi_{e}^{*} \sigma\right)\left(\Delta^{n+1}\right)=\mathcal{A}\left(\varphi_{e}^{*} E, \varphi_{e}^{*} \sigma\right)\left(\partial \Delta^{n+1}\right) .
\end{aligned}
$$


On the other hand, by applying Lemma 6.12 to $\left.\varphi_{e}^{*} \sigma\right|_{\left.\left(\Delta^{n+1}\right)^{(n)}\right)}$, we have

$$
\mathfrak{M}\left(s_{\left.\varphi_{e}^{*} \sigma\right|_{\left(\Delta^{n+1}\right)} ^{(n)}}, 1 ;\left(\Delta^{n+1}\right)^{(n-1)}\right)=\mathcal{A}\left(\varphi_{e}^{*} E, \varphi_{e}^{*} \sigma\right) .
$$

In addition, since $\mathcal{M}_{\varphi_{e}^{*} \sigma^{(n-1)}}=\emptyset$ holds, we can define

$$
\mathfrak{M}\left(s_{\varphi_{e}^{*} \sigma}, 1 ;\left(\Delta^{n+1}\right)^{(n-1)}\right) \in H^{n}\left(\Delta^{n+1},\left(\Delta^{n+1}\right)^{(n-1)}\right)
$$

and

$$
i^{*} \mathfrak{M}\left(s_{\varphi_{e}^{*} \sigma}, 1 ;\left(\Delta^{n+1}\right)^{(n-1)}\right)=\mathfrak{M}\left(s_{\left.\varphi_{e}^{*} \sigma\right|_{\left(\Delta^{n+1}\right)(n)}}, 1 ;\left(\Delta^{n+1}\right)^{(n-1)}\right)
$$

holds, where

$$
i:\left(\left(\Delta^{n+1}\right)^{(n)},\left(\Delta^{n+1}\right)^{(n-1)}\right) \hookrightarrow\left(\Delta^{n+1},\left(\Delta^{n+1}\right)^{(n-1)}\right)
$$

is the inclusion. From the equalities (30), (31), (32), and $i_{*} \partial \Delta^{n+1}=0$, it follows that

$$
\begin{aligned}
\delta \mathcal{A}(E, \sigma)(e)=\left\langle\mathcal{A}\left(\varphi_{e}^{*} E, \varphi_{e}^{*} \sigma\right), \partial \Delta^{n+1}\right\rangle & =\left\langle i^{*} \mathfrak{M}\left(s_{\varphi_{e}^{*} \sigma}, 1 ;\left(\Delta^{n+1}\right)^{(n-1)}\right), \partial \Delta^{n+1}\right\rangle \\
& =\left\langle\mathfrak{M}\left(s_{\varphi_{e}^{*} \sigma}, 1 ;\left(\Delta^{n+1}\right)^{(n-1)}\right), i_{*} \partial \Delta^{n+1}\right\rangle=0 .
\end{aligned}
$$

Thus we have $\delta \mathcal{A}(E, \sigma)=0$.

Proof of Theorem 6.4. Let us take two inductive sections $\sigma_{i}:\left.B^{(n)} \rightarrow \Pi(E)\right|_{B^{(n)}}$ for $i=0,1$, and set $\mathcal{A}_{i}:=\mathcal{A}\left(E, \sigma_{i}\right)$. Let $p: B \times[0,1] \rightarrow B$ the the projection. For each cell $e$ of $B$, let $\varphi_{e}: D^{\text {dim } e} \rightarrow B$ be its characteristic map and fix a trivialization

$$
\tilde{\psi}_{e}:\left(\varphi_{e} \times \mathrm{id}\right)^{*} p^{*} \Pi(E) \rightarrow D^{\operatorname{dim} e} \times[0,1] \times \Pi(X) .
$$

We inductively construct a section $\tilde{\sigma}=\tilde{\sigma}^{(n)}: B^{(n)} \times\left.[0,1] \rightarrow p^{*} \Pi(E)\right|_{B^{(n)}}$ as follows. First let $e \subset B$ be a 0 -cell. We take a section $\tilde{\sigma}^{(0)}: e \times\left.[0,1] \rightarrow p^{*} \Pi(E)\right|_{e}$ such that $\left.\tilde{\sigma}^{(0)}\right|_{e \times\{i\}}=\sigma_{i}^{(0)}$ and the composition

$p_{2} \circ \tilde{\psi}_{e} \circ\left(\varphi_{e} \times \mathrm{id}\right)^{*} \tilde{\sigma}^{(0)}: D_{e}^{0} \times(0,1) \rightarrow\left(\varphi_{e} \times \mathrm{id}\right)^{*} p^{*} \Pi(E) \rightarrow D^{0} \times[0,1] \times \Pi(X) \rightarrow \Pi(X)$

is smooth (in the interior of the domain) and generic, and avoiding the wall. Then we obtain $\tilde{\sigma}^{(0)}:\left.B^{(0)} \rightarrow p^{*} \Pi(E)\right|_{B^{(0)}}$. We next assume that we have constructed $\tilde{\sigma}^{(k-1)}:\left.B^{(k-1)} \rightarrow p^{*} \Pi(E)\right|_{B^{(k-1)}}$ for $k \leq n$ such that $\left.\tilde{\sigma}^{(k-1)}\right|_{e \times\{i\}}=\sigma_{i}^{(k-1)}$ and $\mathcal{M}_{\tilde{\sigma}^{(k-1)}}=\emptyset$. For a $k$-cell $e \subset B$, we take a section $\tilde{\sigma}^{(k)}: e \times\left.[0,1] \rightarrow p^{*} \Pi(E)\right|_{e}$ such that $\left.\tilde{\sigma}^{(k)}\right|_{e \times\{i\}}=\sigma_{i}^{(k)},\left.\tilde{\sigma}^{(k)}\right|_{\bar{e} \backslash e}=\left.\tilde{\sigma}^{(k-1)}\right|_{\bar{e} \backslash e}$, and the composition

$$
p_{2} \circ \tilde{\psi}_{e} \circ\left(\varphi_{e} \times \mathrm{id}\right)^{*} \tilde{\sigma}^{(k)}: D_{e}^{k} \times(0,1) \rightarrow \Pi(X)
$$

is smooth and generic, and avoiding the wall. We can assume that this composition avoids the wall since we assume that $b^{+}(X) \geq n+2$. We now obtain $\tilde{\sigma}^{(k)}: B^{(k)} \rightarrow$ $\left.p^{*} \Pi(E)\right|_{B^{(k)}}$, and thus $\tilde{\sigma}: B^{(n)} \times\left.[0,1] \rightarrow p^{*} \Pi(E)\right|_{B^{(n)}}$. Let us define

$$
\tilde{\mathcal{A}} \in C^{n-1}(B)
$$

by

$$
\tilde{\mathcal{A}}(e):=(-1)^{n-1} \mathcal{A}\left(p^{*} E, \tilde{\sigma}\right)(e \times I)
$$

for each $(n-1)$-cell $e$ of $B$, where $I$ is the 1 -cell of $[0,1]$ equipped with the standard cell structure. We shall show that $\delta \tilde{\mathcal{A}}=\mathcal{A}_{1}-\mathcal{A}_{0}$. Let $R=\mathbb{Z}$ or $\mathbb{Z} / 2$ be the our coefficient, and let us write the basis of $C_{*}(I)$ as $C_{0}(I)=R \cdot 0 \oplus R \cdot 1, C_{1}(I)=R \cdot I$, and write the dual basis as $C^{0}(I)=R \cdot 0^{*} \oplus R \cdot 1^{*}, C^{1}(I)=R \cdot I^{*}$. Let $\Phi: C^{*}(B) \rightarrow$ 
$C^{*+1}(B \times[0,1])$ be the isomorphism given by $\Phi(c):=c \otimes I^{*}$, which commutes with $\delta$. Let us consider an $n$-cell of $B \times[0,1]$ written as

$$
\begin{aligned}
\tilde{e} & =e^{n-1} \otimes I+e_{0}^{n} \otimes 0+e_{1}^{n} \otimes 1 \\
& \in C_{n-1}(B) \otimes C_{1}([0,1]) \oplus C_{n}(B) \otimes C_{0}([0,1]) \cong C_{n}(B \times[0,1]) .
\end{aligned}
$$

Then, since $\mathcal{A}\left(p^{*} E, \tilde{\sigma}\right)(e \times i)=\mathcal{A}_{i}(e)$ holds for each $n$-cell $e$ of $B$, we have

$$
\begin{aligned}
\Phi \tilde{\mathcal{A}}(\tilde{e})=\tilde{\mathcal{A}}\left(e^{n-1}\right) & =(-1)^{n-1} \mathcal{A}\left(p^{*} E, \tilde{\sigma}\right)\left(e^{n-1} \times I\right) \\
& =(-1)^{n-1}\left\{\mathcal{A}\left(p^{*} E, \tilde{\sigma}\right)(\tilde{e})-\mathcal{A}_{0}\left(e_{0}^{n}\right)-\mathcal{A}_{1}\left(e_{1}^{n}\right)\right\} \\
& =(-1)^{n-1}\left\{\mathcal{A}\left(p^{*} E, \tilde{\sigma}\right)(\tilde{e})-\mathcal{A}_{0} \otimes 0^{*}(\tilde{e})-\mathcal{A}_{1} \otimes 1^{*}(\tilde{e})\right\} .
\end{aligned}
$$

It therefore follows that

$$
\Phi \tilde{\mathcal{A}}=(-1)^{n-1}\left(\mathcal{A}\left(p^{*} E, \tilde{\sigma}\right)-\mathcal{A}_{0} \otimes 0^{*}-\mathcal{A}_{1} \otimes 1^{*}\right) .
$$

Using this equality and Proposition 6.2, we have

$$
\begin{aligned}
\Phi \delta \tilde{\mathcal{A}}=\delta \Phi \tilde{\mathcal{A}} & =(-1)^{n-1} \delta\left(\mathcal{A}\left(p^{*} E, \tilde{\sigma}\right)-\mathcal{A}_{0} \otimes 0^{*}-\mathcal{A}_{1} \otimes 1^{*}\right) \\
& =(-1)^{n}\left\{(-1)^{n} \mathcal{A}_{0} \otimes \delta 0^{*}+(-1)^{n} \mathcal{A}_{1} \otimes \delta 1^{*}\right\} \\
& =-\mathcal{A}_{0} \otimes I^{*}+\mathcal{A}_{1} \otimes I^{*}=\Phi\left(-\mathcal{A}_{0}+\mathcal{A}_{1}\right) .
\end{aligned}
$$

Since $\Phi$ is an isomorpshism, we obtain the required equality $\delta \tilde{\mathcal{A}}=\mathcal{A}_{1}-\mathcal{A}_{0}$.

Remark 6.14. In the argument of this subsection, the cohomological invariants given in Definition 5.12 is fruitfully used. This is one of the clear merits of our family virtual neighborhood technique, and here we also mention another good point of it. In the proof of Theorem 6.4 for $i=0,1$, let $\psi_{e, i}: \varphi_{e}^{*} \Pi(E) \rightarrow D_{e}^{n} \times \Pi(X)$ be the trivialization of $\varphi_{e}^{*} \Pi(E) \rightarrow D_{e}^{n}$ used in the construction of $\sigma_{i}$. We note that we do not need to take $\tilde{\psi}_{e}$ to be an extension of $\psi_{e, i}$ : although the composition

$$
p_{2} \circ \tilde{\psi}_{e} \circ \varphi_{e}^{*} \sigma_{i}^{(n)}: D_{e}^{n} \times\{i\} \rightarrow \Pi(X)
$$

is not smooth in general, it does not matter since we here do not consider any cobordism between smooth moduli spaces. This is also one of the advantages of the family virtual neighborhood technique: we do not need to connect by a path between two local trivializations. If one tries to connect the trivializations $\psi_{e, 0}$ and $\psi_{e, 1}$ via a family of trivializations, we have to study $\pi_{0}(\operatorname{Homeo}(\Pi(X) \backslash$ wall $))$, where Homeo $(\cdot)$ denotes the homeomorphism group. Especially in the ASD setting, the structure of the wall is complicated, and hence $\operatorname{Homeo}(\Pi(X) \backslash$ wall $)$ is so. The use of the family virtual neighborhood technique allows us to avoid such a problem.

Remark 6.15. We mention other possibilities of ways to construct gauge theoretic characteristic classes on the classifying space of the diffeomorphism group avoiding family virtual neighborhood technique. A simple way is to abandon $\mathbb{Z}$ or $\mathbb{Z} / 2$ coefficient cohomology and to work over $\mathbb{Q}$. Since any homology class over $\mathbb{Q}$ on any space, say the classifying space, can be represented as a smooth finite dimensional manifold, one may apply usual family gauge theory (and Nakamura's idea described in Section 4) to the representative. For example, D. McDuff [27] describes characteristic classes obtained based on the family Gromov-Witten invariant via this way. Another possibility is to use a model of the classifying space which is a smooth infinite dimensional manifold. (For example, see Kriegl-Michor [21.) However this approach, of course, involves a lot of subtle problems on the counting 
of the moduli space, and the author does not certain whether one can completely avoid family virtual neighborhood technique via this way.

\section{Characteristic Classes as obstruction}

In this section we interpret our characteristic classes $\mathbb{D}$ and $\mathbb{S W}$ as obstructions to some structure on 4-manifold bundles. We first note that these characteristic classes are obstructions to fiberwise connected sum under suitable assumption on $b^{+}$. Let $B$ be a topological space and $X_{i} \rightarrow E_{i} \rightarrow B(i=1,2)$ be fiber bundles of oriented closed 4-manifolds $X_{i}$. Given sections $\mathcal{S}_{i}: B \rightarrow E_{i}$, if the normal bundle of $\mathcal{S}_{1}(B)$ in $E_{1}$ is isomorphic to that of $\mathcal{S}_{2}(B)$ in $E_{2}$ fiber-preservingly and fiber-orientationreversingly, we can define the fiberwise connected sum $X_{1} \# X_{2} \rightarrow E_{1} \#_{f} E_{2} \rightarrow B$ by considering the connected sum $\left(E_{1}\right)_{b} \#\left(E_{2}\right)_{b}$ along small disklike neighborhoods of $\mathcal{S}_{i}(b)$ for each $b \in B$. We call such sections $\mathcal{S}_{i}$ compatible sections. Let $\mathfrak{P}_{i}, \mathfrak{s}_{i}$, and $\mathcal{O}_{i}$ be the isomorphism class of an $S O(3)$-bundle with $w_{2}\left(\mathfrak{P}_{i}\right) \neq 0$, the isomorphism class of a $\operatorname{spin}^{c}$ structure, and a homology orientation on $X_{i}$ respectively. They define data $\mathfrak{P}=\mathfrak{P}_{1} \# \mathfrak{P}_{2}, \mathfrak{s}=\mathfrak{s}_{1} \# \mathfrak{s}_{2}$, and $\mathcal{O}=\mathcal{O}_{1} \# \mathcal{O}_{2}$ on $X=X_{1} \# X_{2}$, and we call the relations between $\mathfrak{P}, \mathfrak{s}, \mathcal{O}$ and $\mathfrak{P}_{i}, \mathfrak{s}_{i}, \mathcal{O}_{i}$ the connected sum relations. The characteristic classes $\mathbb{D}$ and $\mathbb{S} \mathbb{W}$ obstruct the fiberwise connected sum as follows. We note that D. Ruberman has pointed this kind of phenomena out as Theorem 3.3 in 39 .

Theorem 7.1. Let $X$ be an oriented closed smooth 4-manifold, $B$ be a $C W$ complex, $E \rightarrow B$ be a continuous $X$-bundle, and $n$ be a non-negative integer. Assume that $b^{+}(X) \geq n+2$ and $B$ has finitely many $n$-cells, and also suppose that either

(1) $d(\mathfrak{P})=-n$ for the isomorphism class $\mathfrak{P}$ of an $S O(3)$-bundle on $X$ with $w_{2}(\mathfrak{P}) \neq 0$, the structure group of $E$ reduces to $G=\operatorname{Diff}(X, \mathfrak{P})$ or $\operatorname{Diff}(X, \mathfrak{P}, \mathcal{O})$ for some homology orientation $\mathcal{O}$, and $\mathbb{D}(E) \neq 0$ or

(2) $d(\mathfrak{s})=-n$ for the isomorphism class $\mathfrak{s}$ of a spin ${ }^{c}$ structure on $X$, the structure group of $E$ reduces to $G=\operatorname{Diff}(X, \mathfrak{s})$ or $\operatorname{Diff}(X, \mathfrak{s}, \mathcal{O})$ for some homology orientation $\mathcal{O}$, and $\mathbb{S W}(E) \neq 0$.

Then, there are no bundles of oriented closed 4-manifolds $X_{i} \rightarrow E_{i} \rightarrow B(i=$ $1,2)$ with structure group $G_{i}$ satisfying that $E=E_{1} \#_{f} E_{2}$ along some compatible sections $\mathcal{S}_{i}: B \rightarrow E_{i}$ and that $b^{+}\left(X_{i}\right)>n$. Here $G_{i}$ is given as $\operatorname{Diff}\left(X_{i}, \mathfrak{P}_{i}\right)$, $\operatorname{Diff}\left(X_{i}, \mathfrak{P}_{i}, \mathcal{O}_{i}\right), \operatorname{Diff}\left(X_{i}, \mathfrak{s}_{i}\right)$, or $\operatorname{Diff}\left(X_{i}, \mathfrak{s}_{i}, \mathcal{O}_{i}\right)$ corresponding to $G$ for some $\mathfrak{P}_{i}$, $\mathfrak{s}_{i}$, or $\mathcal{O}_{i}$ satisfying the connected sum relations.

Proof. Let us write $\mathbb{A}$ for $\mathbb{D}$ and $\mathbb{S W}$ as Section 6 . Assume that there exist $X_{i} \rightarrow$ $E_{i} \rightarrow B(i=1,2)$ with structure group $G_{i}$ satisfying that $E=E \#_{f} E_{2}$ along some sections $\mathcal{S}_{i}: B \rightarrow E_{i}$ and that $b^{+}\left(X_{i}\right)>n$. As in the usual vanishing theorem of the Donaldson invariant or Seiberg-Witten invariant for connected sum, consider a sequence of fiberwise metrics $g_{i, b, m} \in \operatorname{Met}\left(\left(E_{i}\right)_{b}\right)(b \in B, m \in \mathbb{N})$ and $g_{b, m}=g_{1, b, m} \# g_{2, b, m} \in \operatorname{Met}\left(E_{b}\right)$ such that $g_{b, m}$ has a neck whose radius converges to 0 as $m \rightarrow \infty$ and $g_{i, b, m}$ is constant with respect to $m$ on the complement of a neighborhood of the disk used to define the neck. If we work on the ASD setting, we can define inductive sections $\sigma_{i, m}:\left.B^{(n)} \rightarrow \Pi\left(E_{i}\right)\right|_{B^{(n)}}$ as $\sigma_{i, m}(b)=g_{i, b, m}$ by taking suitable $g_{i, b, m}$ in advance. If we work on the SW setting, we take inductive sections $\sigma_{i, m}:\left.B^{(n)} \rightarrow \Pi\left(E_{i}\right)\right|_{B^{(n)}}$ so that $\sigma_{i, m}(b)$ vanishes near the disk used to define the neck, $\sigma_{i, m}(b)$ is constant with respect to $m$ on the complement of the disk, and $\pi\left(\sigma_{i, m}(b)\right)=g_{i, b, m}$ holds, where $\pi: \Pi\left(\left(E_{i}\right)_{b}\right) \rightarrow \operatorname{Met}\left(\left(E_{i}\right)_{b}\right)$ is the projection. 
The inductive sections $\sigma_{i, m}$ define the limiting inductive sections $\sigma_{i, \infty}: B^{(n)} \rightarrow$ $\left.\Pi\left(E_{i}\right)\right|_{B^{(n)}}$ by an obvious way. Let us define $\sigma_{m}(b):=\sigma_{1, m}(b) \# \sigma_{2, m}(b)$. This new section $\sigma_{m}:\left.B^{(n)} \rightarrow \Pi(E)\right|_{B^{(n)}}$ is also an inductive section. Assume that, for any $m$, there exists an $n$-cell of $B$ such that the parameterized moduli space with respect to $\sigma_{m}$ on it is not empty. Because of our finiteness assumption on $n$-cells of $B$, without loss of generality, we may assume that there exists an $n$-cell $e$ of $B$ such that, for any $m>>$, the parameterized moduli space with respect to $\sigma_{m}$ on $e$ is not empty. Let $\varphi_{e}: D_{e}^{n} \rightarrow B$ be the characteristic map of $e$. By considering limit and removing the singularity, we get an element of the parameterized moduli pace with respect to $\sigma_{i, \infty}$ on $e$ for both $i=1,2$. On the other hand, by the definition of inductive section, there exists trivializations $\psi_{e, i}: \varphi_{e}^{*} \Pi\left(E_{i}\right) \rightarrow D_{e}^{n} \times \Pi\left(X_{i}\right)$ such that $\sigma_{i, \infty}$ is smooth, generic and avoiding reducibles via $\psi_{e, i}$. The parameterized moduli spaces with respect to $\sigma_{i, \infty}$ on $e$ therefore admit smooth structure via $\psi_{e, i}$, and hence one of them is empty because of formal dimension. This contradicts the existence of the limiting elements discussed above. This argument implies that there exists $m$ such that the parameterized moduli space with respect to $\sigma_{m}$ is empty. Thus we have $\mathcal{A}\left(E, \sigma_{m}\right)=0$, and hence $\mathbb{A}(E)=0$.

We next mention that $\mathbb{S W}$ relates to families of positive scalar curvature metrics, as in D. Ruberman [40] and [20] by the author. For a given 4-manifold $X$, we denote by $\operatorname{PSC}(X)$ the space of positive scalar curvature metrics on $X$. For a fiber bundle $X \rightarrow E \rightarrow B$ with structure group $\operatorname{Diff}(X)$, the diffeomorphism group of $X$, on a topological space $B$, since $\operatorname{Diff}(X)$ acts on $\operatorname{PSC}(X)$, we get a fiber bundle $\operatorname{PSC}(E) \rightarrow B$ whose fiber is $\operatorname{PSC}(X)$. The following theorem generalizes Proposition 2.4 in [20].

Theorem 7.2. Let $X$ be an oriented closed smooth 4-manifold, $\mathfrak{s}$ be the isomorphism class of a spin ${ }^{c}$ structure on $X, B$ be a $C W$ complex, and $n$ be a nonnegative number. Assume that $\operatorname{PSC}(X) \neq \emptyset, b^{+}(X) \geq n+2, d(\mathfrak{s})=-n$, and $B^{(n)}$ is compact. Let $E \rightarrow B$ be a bundle of $X$ with structure group $\operatorname{Diff}(X, \mathfrak{s})$ or $\operatorname{Diff}(X, \mathfrak{s}, \mathcal{O})$ for some homology orientation $\mathcal{O}$. Then, if $\mathbb{S W}(E) \neq 0$, there is no section of $\left.\operatorname{PSC}(E)\right|_{B^{(n)}} \rightarrow B^{(n)}$. In particular, $\pi_{i}(\operatorname{PSC}(X)) \neq 0$ holds for at least one $i \in\{0, \ldots, n-1\}$.

Proof. We first consider the case that either $c_{1}(\mathfrak{s})^{2}>0$, or $c_{1}(\mathfrak{s})^{2}=0$ and $c_{1}(\mathfrak{s})$ is not torsion. In these cases, $\operatorname{PSC}(X)$ does not intersects with the wall. We can therefore construct an inductive section $\sigma:\left.B^{(n)} \rightarrow \Pi(E)\right|_{B^{(n)}}$ so that $\sigma$ factors through the inclusion $\operatorname{PSC}(E) \hookrightarrow \operatorname{Met}(X) \hookrightarrow \Pi(E)$. Since (unperturbed) SeibergWitten equations has no solution with respect to a positive scalar curvature metrics, we have $\mathcal{S} \mathcal{W}(E, \sigma)=0$, and hence $\mathbb{S W}(E)=0$. Here $\mathcal{S W}(\cdot)$ is the cochain corresponding to $\mathcal{A}(\cdot)$ in Subsection 6.1 for the SW setting. For other possibilities on $c_{1}(\mathfrak{s})$, one can easily modify the above argument. (In particular, for the case that $c_{1}(\mathfrak{s})$ is torsion, we have to consider perturbed Seiberg-Witten equations. For the proof of the case, we need the compactness assumption on $B^{(n)}$.) See the proof of Proposition 2.4 in 20] for the modification.

\section{Calculations}

In this section we give some calculations of our characteristic classes. In Subsection 8.2 we develop a version of higher-dimensional wall-crossing and use it to calculations of $\mathbb{S W}$. To give more subtle examples, in Subsections 8.3 and 8.4 we 
combine an argument of D. Ruberman's 38] and calculations given in 20] and in Subsection 8.2. In Subsection 8.5 we mention a calculation of $\mathbb{D}$ obtained from D. Ruberman's result [38. In Subsections 8.2 to 8.4 we use the idea of combining the higher-dimensional mapping torus and Seiberg-Witten equations due to N. Nakamura 33 .

8.1. Almost localized harmonic forms. The purpose of this subsection is to describe a tool used in Subsection 8.2 harmonic forms on a manifold given as a connected sum of several manifolds which is almost localized on one of connected sum components in $L^{2}$-sense. This is obtained by mimicking the argument of the additivity of Fredholm index of linear elliptic operators. We mainly refer to Donaldson [10] in this subsection.

For $i=1,2$, let $\left(X_{i}, g_{i}\right)$ be oriented Riemannian manifolds with $b_{1}\left(X_{i}\right)=0$ and $Y$ be an oriented closed Riemannian 3-manifold. Assume that $X_{i}$ has one end which is isometric to the cylinder $Y \times[0, \infty)$ with the product metric, and that the boundary of the complement of the end in $X_{1}$ coincides with $Y$ with the given orientation and that in $X_{2}$ do $-Y$ : the opposite orientation. For $S>0$, let $X_{i}(S)$ be the compact 4-manifold obtained by cutting the end $Y \times(S, \infty)$ from $X_{i}$. For $T>0$, by identifying $Y \times\{t\}$ in $X_{1}$ and $-Y \times\{2 T-t\}$ in $X_{2}$, we obtain a closed 4manifold $X_{T}$, often denoted by $X$. The closed 4-manifold $X_{T}$ contains a cylindrical part which is isometric to $Y \times[0,2 T]$. Let $D_{i}$ and $D=D_{T}$ be the elliptic operators given by either

$$
\begin{aligned}
& D_{i}:=d+d^{*}: \Omega^{\text {even }}\left(X_{i}\right) \rightarrow \Omega^{\text {odd }}\left(X_{i}\right), \\
& D=D_{T}:=d+d^{*}: \Omega^{\text {even }}\left(X_{T}\right) \rightarrow \Omega^{\text {odd }}\left(X_{T}\right)
\end{aligned}
$$

or

$$
\begin{aligned}
& D_{i}:=d+\left(d^{+}\right)^{*}: \Omega^{0}\left(X_{i}\right) \oplus \Omega^{+}\left(X_{i}\right) \rightarrow \Omega^{1}\left(X_{i}\right), \\
& D=D_{T}:=d+\left(d^{+}\right)^{*}: \Omega^{0}\left(X_{T}\right) \oplus \Omega^{+}\left(X_{T}\right) \rightarrow \Omega^{1}\left(X_{T}\right) .
\end{aligned}
$$

Recall that the $L^{2}$-orthogonal decomposition $\Omega^{+}(X)=\mathcal{H}^{+} \oplus \operatorname{Im} d^{+}$, where $\mathcal{H}^{+}$is the space of self-dual harmonic 2-forms. Because of this decomposition, if $\mu \in \Omega^{+}(X)$ satisfies $\left(d^{+}\right)^{*} \mu=0$, then $\mu \in \mathcal{H}^{+}$holds. In fact, for our purpose of Subsection 8.2. it is sufficient to consider only the latter operators, which we call the AtiyahHitchin-Singer operators, rather than the de Rham operators. However the whole arguments in this subsection are parallel to both operators, and so we describe this almost localization phenomena for both operators. On the cylidrical part, these operators admit the decomposition

$$
d+d^{*}=\frac{\partial}{\partial t}+L_{Y}
$$

where $t$ is the coordinate of $[0, \infty)$ (or some finite length interval) and $L_{Y}$ is a linear elliptic formally self-adjoint operator on $Y$. (For example, see Section 9.2 of Melrose [28] for the de Rham operators and Subsection 3.1 of Donaldson [10] for the Atiyah-Hitchin-Singer operators.) In fact, $L_{Y}$ has non-trivial kernel, and we therefore use weighted Sobolev spaces. Let $\delta>0$ be a sufficiently small positive number such that $[-\delta, \delta] \backslash\{0\}$ contains no spectrum of both $D_{i}$. Fix a smooth positive function $W_{i}$ on $X_{i}$ whose restriction on the cylindrical end coincides with $e^{\delta t}$. We take $W_{i}$ so that $W_{i} \geq 1$ on $X_{i}(1)$, and define $\|f\|_{L_{k, \delta}^{2}}:=\left\|W_{i} f\right\|_{L_{k}^{2}}$. We 
simply write $L_{k, \delta}^{2}\left(X_{i}\right)$ for our functional space if the bundle which we consider is clear. We now get the extended continuous operator

$$
D_{i}: L_{1, \delta}^{2}\left(X_{i}\right) \rightarrow L_{\delta}^{2}\left(X_{i}\right)
$$

which is Fredholm. Henceforth, the notations $\operatorname{Ker} D_{i}$ and Coker $D_{i}$ denote the kernel and the cokernel of this extended operator respectively. The operator $D_{i}$ is positively weighted, and hence the adjoint $D_{i}^{*}$ is negatively weighted. Therefore Coker $D_{i}$ is isomorphic to the space of $L^{2}$-solutions to

$$
D_{i}^{*}=d+d^{*}: \Omega^{\text {odd }}\left(X_{i}\right) \rightarrow \Omega^{\text {even }}\left(X_{i}\right) .
$$

If we consider the de Rham operators, thanks to Proposition 4.9 in Atiyah-PatodiSinger [3], the space of $L^{2}$-solutions to $D_{i}^{*}$ is isomorphic to $\hat{H}^{1}\left(X_{i} ; \mathbb{R}\right) \oplus \hat{H}^{3}\left(X_{i} ; \mathbb{R}\right)$, where $\hat{H}^{*}\left(X_{i} ; \mathbb{R}\right)$ is the image of $H^{*}(Y ; \mathbb{R}) \rightarrow H^{*}\left(X_{i}(0) ; \mathbb{R}\right)$. This space vanishes since we assumed that $b_{1}\left(X_{i}\right)=0$. Similarly, if we consider the Atiyah-HitchinSinger operators, we can use the adjoint version of Proposition 3.15 in Donaldson [10. It implies that $\operatorname{Ker} D_{i}^{*} \cong H^{1}\left(X_{i} ; \mathbb{R}\right)=0$. Thus we have Coker $D_{i}=0$ in both cases, and hence there exists a bounded right inverse of $D_{i}$. We here use a concrete right inverse $Q^{X_{i}}: L_{\delta}^{2}\left(X_{i}\right) \rightarrow L_{1, \delta}^{2}\left(X_{i}\right)$ : the projection to the $L_{1, \delta^{-}}^{2}$ orthogonal complement of $\operatorname{Ker} D_{i}$. Take a positive number $T_{1}>0$ with $T \geq T_{1}$. Fix cut-off functions $\rho_{1, T_{1}}^{i}: X_{i} \rightarrow[0,1]$ satisfying:

- $\rho_{1, T_{1}}^{1}$ is supported on $X_{1}\left(3 T_{1} / 2\right)$ and $\rho_{1, T_{1}}^{1}=1$ on $X_{1}\left(T_{1} / 2\right)$,

- $\left|\nabla \rho_{1, T_{1}}^{1}\right| \leq 2 / T_{1}$, and

- $\rho_{1, T_{1}}^{1}+\rho_{1, T_{1}}^{2}=1$.

Using a way given in Subsection 3.3 in [10, we can construct a right inverse $Q_{1, T_{1}}^{X}$ of $D$ as follows. We first define $P_{1, T_{1}}: L^{2}(X) \rightarrow L_{1, \delta}^{2}(X)$ by

$$
P_{1, T_{1}}(\eta):=\rho_{1, T_{1}}^{1} Q^{X_{1}}\left(\eta_{1}\right)+\rho_{1, T_{1}}^{2} Q^{X_{2}}\left(\eta_{2}\right)
$$

where $\eta_{1}$ is the restriction of $\eta$ to $X_{1}\left(2 T_{1}\right) \subset X$ and $\eta_{2}$ is that of $\eta$ to $X_{2}(2 T) \subset X$, extended by zero to the rest of $X$. Then we obtain an estimate $\left\|D P_{1, T_{1}}-\mathrm{id}\right\|_{\text {op }}<$ $C_{1} / T_{1}$, where $\|\cdot\|_{\text {op }}$ denotes the operator norm and $C_{1}$ is a universal constant. By taking $T_{1}$ as $T_{1}>C_{1}$, we can define $\left(D P_{1, T_{1}}\right)^{-1}$ using the Neumann series, and so can define $Q_{1, T_{1}}^{X}:=P\left(D P_{1, T_{1}}\right)^{-1}$. By construction, $\left\|Q_{1, T_{1}}^{X}\right\|_{\text {op }}$ continuously depends on $g_{1}$ and $g_{2}$ since $\left\|Q^{X_{i}}\right\|_{\text {op }}$ continuously do on $g_{i}$. Henceforth in this subsection we fix $T \geq T_{1}>C_{1}$. Let us define a map

$$
\Theta_{1, T_{1}}: \operatorname{Ker} D_{1} \oplus \operatorname{Ker} D_{2} \rightarrow \operatorname{Ker} D
$$

by

$$
\left(\mu_{1}, \mu_{2}\right) \mapsto \theta_{1, T_{1}}\left(\mu_{1}, \mu_{2}\right)-Q_{1, T_{1}}^{X} D \theta_{1, T_{1}}\left(\mu_{1}, \mu_{2}\right),
$$

where $\theta_{1, T_{1}}\left(\mu_{1}, \mu_{2}\right):=\rho_{1, T_{1}}^{1} \mu_{1}+\rho_{1, T_{1}}^{2} \mu_{2}$. Similarly, for $T \geq T_{2}>C_{1}$, by taking $\rho_{2, T_{2}}^{1}$ and $\rho_{2, T_{2}}^{2}$, we can define $Q_{2, T_{2}}^{X}, \theta_{2, T_{2}}$, and

$$
\Theta_{2, T_{2}}: \operatorname{Ker} D_{1} \oplus \operatorname{Ker} D_{2} \rightarrow \operatorname{Ker} D \text {. }
$$

Definition 8.1. Let $T \geq T_{1}, T_{2}>C_{1}$. For $\mu \in \operatorname{Ker} D_{1}$, we call $\Theta_{1, T_{1}}(\mu, 0) \in$ $\operatorname{Ker} D_{T}$ the almost localized harmonic form on $X_{1}$ arising from $\mu$ with respect to $T$ and $T_{1}$ (and $g_{1}, g_{2}$ ). We briefly write $\check{\mu}$ for $\Theta_{1, T_{1}}(\mu, 0)$ if there is no confusion. Similarly we define almost localized harmonic forms on $X_{2}$ using $\Theta_{2, T_{2}}$. In particular, when we consider the Atiyah-Hitchin-Singer operators and $\mu \in \operatorname{Ker} D_{1} \cap \Omega^{+}\left(X_{i}\right)$, 
we also call $\check{\mu}$ the almost localized self-dual harmonic form arising from $\mu$ to emphasize self duality.

Lemma 8.2. For $\left(\mu_{1}, \mu_{2}\right) \in \operatorname{Ker} D_{1} \oplus \operatorname{Ker} D_{2}$,

$$
\left\|Q_{i, T_{i}}^{X} D \theta_{i, T_{i}}\left(\mu_{1}, \mu_{2}\right)\right\|_{L_{\delta}^{2}} \leq \frac{C_{2}}{T_{i}}\left(\left\|\mu_{1}\right\|_{L_{\delta}^{2}}+\left\|\mu_{2}\right\|_{L_{\delta}^{2}}\right)
$$

holds, where $C_{2}=C_{2}\left(g_{1}, g_{2}\right)$ is a constant continuously depending on $g_{1}$ and $g_{2}$.

Proof. See Subsection 3.3 in [10. The continuous dependence of $C_{2}\left(g_{1}, g_{2}\right)$ follows from that of $\left\|Q_{i, T_{i}}^{X}\right\|_{\mathrm{op}}$.

The interaction between almost localized harmonic forms on $X_{1}$ and $X_{2}$ is as follows:

Lemma 8.3. Assume that $4 T \geq 3\left(T_{1}+T_{2}\right)$ and that $T_{1}, T_{2} \geq 1$. Let $\mu_{i} \in \operatorname{Ker} D_{i}$ for $i=1,2$. Then, for $\check{\mu}_{1}:=\Theta_{1, T_{1}}\left(\mu_{1}, 0\right)$ and $\check{\mu}_{2}:=\Theta_{2, T_{2}}\left(0, \mu_{2}\right)$, we have

$$
\left|\left(\check{\mu}_{1}, \check{\mu}_{2}\right)_{L^{2}}\right| \leq \frac{C_{3}}{\min \left\{T_{1}, T_{2}\right\}}\left(\left\|\mu_{1}\right\|_{L_{\delta}^{2}}+\left\|\mu_{2}\right\|_{L_{\delta}^{2}}\right)^{2}
$$

where $C_{3}=C_{3}\left(g_{1}, g_{2}\right)$ is a constant continuously depending on $g_{1}$ and $g_{2}$.

Proof. Note that $\|f\|_{L^{2}} \leq\|f\|_{L_{\delta}^{2}}$ holds for a general $f$, and also note that

$$
\left(\rho_{1, T_{1}}^{1} \mu_{1}, \rho_{2, T_{2}}^{2} \mu_{2}\right)_{L^{2}}=0
$$

follows from $4 T \geq 3\left(T_{1}+T_{2}\right)$. We therefore have

$$
\begin{aligned}
\left|\left(\check{\mu}_{1}, \check{\mu}_{2}\right)_{L^{2}}\right| \leq & \frac{C_{2}}{T_{1}}\left(\left\|\mu_{1}\right\|_{L_{\delta}^{2}}+\left\|\mu_{2}\right\|_{L_{\delta}^{2}}\right)\left\|\mu_{2}\right\|_{L_{\delta}^{2}} \\
& \left.+\frac{C_{2}}{T_{2}}\left\|\mu_{1}\right\|_{L_{\delta}^{2}}\left\|\mu_{1}\right\|_{L_{\delta}^{2}}+\left\|\mu_{2}\right\|_{L_{\delta}^{2}}\right)+\frac{C_{2}^{2}}{T_{1} T_{2}}\left(\left\|\mu_{1}\right\|_{L_{\delta}^{2}}+\left\|\mu_{2}\right\|_{L_{\delta}^{2}}\right)^{2}
\end{aligned}
$$

using Lemma 8.2. Thus we obtain the inequality (34) for suitable $C_{3}$.

The interaction between almost localized harmonic forms on a common connected sum component is as follows:

Lemma 8.4. Fix an $L^{2}$-orthonormal basis $\left\{\eta_{j}\right\}_{j}$ of Ker $D_{1}$. Then, there exists a family of positive numbers $\epsilon_{1}\left(T_{1}\right)>0$ with $\epsilon_{1}\left(T_{1}\right) \rightarrow 0$ as $T_{1} \rightarrow \infty$ satisfying the following conditions:

- $\epsilon_{1}\left(T_{1}\right)=\epsilon_{1}\left(T_{1} ; g_{1}, g_{2},\left\{\eta_{j}\right\}_{j}\right)$ continuously depends on $T_{1}, g_{1}, g_{2}$, and $\left\{\eta_{j}\right\}_{j}$.

- Let $\mu, \mu^{\prime} \in \operatorname{Ker} D_{1}$. Then, for $\check{\mu}:=\Theta_{1, T_{1}}(\mu, 0)$ and $\check{\mu}^{\prime}:=\Theta_{1, T_{1}}\left(\mu^{\prime}, 0\right)$, we have

$$
\left|\left(\check{\mu}, \check{\mu}^{\prime}\right)_{L^{2}}-\left(\mu, \mu^{\prime}\right)_{L^{2}}\right| \leq \epsilon_{1}\left(T_{1}\right)\|\mu\|_{L_{\delta}^{2}}\left\|\mu^{\prime}\right\|_{L_{\delta}^{2}} .
$$

Proof. For any $\mu \in \operatorname{Ker} D_{1}$, we have

$$
\left\|\mu-\theta_{1, T_{1}}(\mu, 0)\right\|_{L^{2}}^{2} \leq \int_{Y \times\left[T_{1} / 2, \infty\right)}|\mu|^{2} \leq\left(2 \sum_{j} \int_{Y \times\left[T_{1} / 2, \infty\right)}\left|\eta_{j}\right|^{2}\right)\|\mu\|_{L^{2}}^{2} .
$$

Note that an element of $\operatorname{Ker} D_{i}$ is smooth, and so is $\eta_{j}$. Hence

$$
\epsilon_{1}\left(T_{1}\right):=2 \max \left\{2 \sum_{j} \int_{Y \times\left[T_{1} / 2, \infty\right)}\left|\eta_{j}\right|^{2},\left(\frac{C_{2}}{T_{1}}\right)^{2}\right\}
$$


continuously depends on $T_{1}, g_{1}, g_{2}$, and $\left\{\eta_{j}\right\}_{j}$. In addition, for $\mu, \mu^{\prime} \in \operatorname{Ker} D_{1}$, we obtain the estimate

$$
\begin{aligned}
& \left|\left(\check{\mu}, \check{\mu}^{\prime}\right)_{L^{2}}-\left(\mu, \mu^{\prime}\right)_{L^{2}}\right| \\
\leq & \left|\left(\check{\mu}-\theta_{1, T_{1}}(\mu, 0), \check{\mu}^{\prime}-\theta_{1, T_{1}}\left(\mu^{\prime}, 0\right)\right)_{L^{2}}\right|+\left|\left(\theta_{1, T_{1}}(\mu, 0)-\mu, \theta_{1, T_{1}}\left(\mu^{\prime}, 0\right)-\mu^{\prime}\right)_{L^{2}}\right| \\
= & \left|\left(Q_{1, T_{1}}^{X} D \theta_{1, T_{1}}(\mu, 0), Q_{1, T_{1}}^{X} D \theta_{1, T_{1}}\left(\mu^{\prime}, 0\right)\right)_{L^{2}}\right| \\
& +\left|\left(\theta_{1, T_{1}}(\mu, 0)-\mu, \theta_{1, T_{1}}\left(\mu^{\prime}, 0\right)-\mu^{\prime}\right)_{L^{2}}\right| \\
\leq & \epsilon_{1}\left(T_{1}\right)\|\mu\|_{L_{\delta}^{2}}\left\|\mu^{\prime}\right\|_{L_{\delta}^{2}}
\end{aligned}
$$

from Lemma 8.2 and the inequality (35).

We remark a family version of the whole construction. Let $B$ be a compact topological space and $\left\{g_{i}^{b}\right\}_{b \in B}$ be a continuous family of Riemannian metrics on $X_{i}$ whose restriction on the end of $X_{i}$ is constant with respect to $b \in B$ and is isometric to $Y \times[0, \infty)$ like $g_{i}$ above. Then, for each $T>0$, we get a family of Riemannian metrics $\left\{g_{T}^{b}\right\}$ on $X$ by gluing $\left(X_{1}, g_{1}^{b}\right)$ and $\left(X_{2}, g_{2}^{b}\right)$. Since metrics on $Y$ is constant, $L_{Y}$ is also a constant operator. Thus we can take a weight $\delta>0$ uniformly with respect to $b \in B$. For this $\delta$ and $T_{1}, T_{2}$ with $T \geq T_{1}, T_{2}>C_{1}$, we can define $\Theta_{i, T_{i}}^{b}: \operatorname{Ker} D_{1}^{b} \oplus \operatorname{Ker} D_{2}^{b} \rightarrow \operatorname{Ker} D_{T}^{b}$ as $\Theta_{i, T_{i}}$ above, where $D_{i}^{b}=d+d^{*} g_{i}^{b}$ and $D_{T}^{b}=d+d^{*} g_{T}^{b}$. Of course, the 4-dimensional operators $D_{i}^{b}$ depends on $b$. However $\bigsqcup_{b \in B} \operatorname{Ker} D_{i}^{b} \rightarrow B$ forms a vector bundle as follows. We first consider the de Rham operators. In this case $\operatorname{Ker} D_{i}^{b}$ is isomorphic to the space of extended $L^{2}$-solutions to $D_{i}^{b}$ in the sense of Atiyah-Patodi-Singer [3] since $D_{i}^{b}$ is equipped with a positive weight. This space is isomorphic to $H^{0}\left(X_{i} ; \mathbb{R}\right) \oplus H^{2}\left(X_{i} ; \mathbb{R}\right) \oplus H^{4}\left(X_{i} ; \mathbb{R}\right)$. Similarly, if we consider the Atiyah-Hitchin-Singer operators, we have $\operatorname{Ker} D_{i}^{b} \cong H^{+}\left(X_{i} ; \mathbb{R}\right)$, which is the adjoint version of Proposition 3.15 in Donaldson [10]. Hence dim $\operatorname{Ker} D_{i}^{b}$ is constant with respect to $b \in B$ and $\bigsqcup_{b \in B} \operatorname{Ker} D_{i}^{b} \rightarrow B$ is a vector bundle for both cases. Suppose that $\bigsqcup_{b \in B} \operatorname{Ker} D_{i}^{b} \rightarrow B$ is trivial (for example $B$ is contractible), and take a global section $\left\{\eta_{j}^{b}\right\}_{j, b}$ of the frame bundle of $\bigsqcup_{b \in B} \operatorname{Ker} D_{1}^{b} \rightarrow B$ respecting the metric for each fiber. By replacing each of $C_{2}, C_{3}$, and $\epsilon_{1}\left(T_{1}\right)$ in the above argument with the maximum of it with respect to $b \in B$, we have the following lemmas corresponding to Lemmas 8.3 and 8.4

Lemma 8.5. Assume that $4 T \geq 3\left(T_{1}+T_{2}\right)$ and that $T_{1}, T_{2} \geq 1$. Then there exists a constant $C_{3}>0$ such that the following holds. Let $\left\{\mu_{i}^{b}\right\}_{b \in B}$ be a section of $\bigsqcup_{b \in B} \operatorname{Ker} D_{i}^{b} \rightarrow B$ for $i=1,2$. Then, for $\check{\mu}_{1}^{b}:=\Theta_{1, T_{1}}^{b}\left(\mu_{1}^{b}, 0\right)$ and $\check{\mu}_{2}^{b}:=\Theta_{2, T_{2}}^{b}\left(0, \mu_{2}^{b}\right)$, we have

$$
\left|\left(\check{\mu}_{1}^{b}, \check{\mu}_{2}^{b}\right)_{L^{2}}\right| \leq \frac{C_{3}}{\min \left\{T_{1}, T_{2}\right\}}\left(\left\|\mu_{1}^{b}\right\|_{L_{\delta}^{2}}+\left\|\mu_{2}^{b}\right\|_{L_{\delta}^{2}}\right)^{2} .
$$

Lemma 8.6. There exists a family of positive numbers $\epsilon_{1}\left(T_{1}\right)>0$ with $\epsilon_{1}\left(T_{1}\right) \rightarrow 0$ as $T_{1} \rightarrow \infty$ such that the following holds. Let $\left\{\mu^{b}\right\}_{b \in B},\left\{\mu^{\prime b}\right\}_{b \in B}$ be sections of $\bigsqcup_{b \in B} \operatorname{Ker} D_{1}^{b} \rightarrow B$. Then, for $\check{\mu}^{b}:=\Theta_{1, T_{1}}^{b}\left(\mu^{b}, 0\right)$ and $\check{\mu}^{\prime b}:=\Theta_{1, T_{1}}^{b}\left(\mu^{\prime b}, 0\right)$, we have

$$
\left|\left(\check{\mu}^{b}, \check{\mu}^{\prime b}\right)_{L^{2}}-\left(\mu^{b}, \mu^{\prime b}\right)_{L^{2}}\right| \leq \epsilon_{1}\left(T_{1}\right)\left\|\mu^{b}\right\|_{L_{\delta}^{2}}\left\|\mu^{\prime b}\right\|_{L_{\delta}^{2}} .
$$

Remark 8.7. In the argument for families, even if metrics on $Y$ varies, in fact we can take uniform $\delta$. This is because $B$ is compact and $\operatorname{dim} \operatorname{Ker} L_{Y}$ is constant. (See the above argument for $\operatorname{dim} \operatorname{Ker} D_{i}$.) 
Remark 8.8. We can work with a negative weight for $D_{i}$. For example, let us consider the de Rham operators. In this case Coker $D_{i}$ is isomorphic to the extended $L^{2}$-solutions to this operator, and is also isomorphic to $H^{1}\left(X_{i} ; \mathbb{R}\right) \oplus H^{3}\left(X_{i} ; \mathbb{R}\right)$. Therefore, if we assume $b_{1}\left(X_{i}\right)=0$, the same argument works.

8.2. SW for connected sum with $n\left(S^{2} \times S^{2}\right)$. We fix $n>0$ in this subsection. We shall calculate $\mathbb{S W}$ for a bundle whose fiber is a 4-manifold given as the connected sum with a given 4-manifold and $N:=n S^{2} \times S^{2}$. Set $\Sigma_{1}=S^{2} \times\{\mathrm{pt}\}$ and $\Sigma_{2}=$ $\{\mathrm{pt}\} \times S^{2}$. For each $i \in\{1, \ldots, n\}$, let $f_{i} \in \operatorname{Diff}^{+}\left(S^{2} \times S^{2}\right)$ be an orientation preserving diffeomorphism admitting a fixed 4-disk, and assume that the induced map $\left(f_{i}\right)_{*}$ on $H_{2}\left(S^{2} \times S^{2}\right)$ is given by either

$$
\left(\begin{array}{cc}
-1 & 0 \\
0 & -1
\end{array}\right) \text { or } \quad\left(\begin{array}{cc}
0 & -1 \\
-1 & 0
\end{array}\right)
$$

where we take $\left\{\left[\Sigma_{1}\right],\left[\Sigma_{2}\right]\right\}$ as the basis of $H_{2}\left(S^{2} \times S^{2}\right)$. We write each connected sum component of $N=\#_{i=1}^{n} S^{2} \times S^{2}$ as $N_{i}$, namely $N=\#_{i=n}^{n} N_{i}$. Regard $f_{i}$ as a diffeomorphism on $N_{i}$, and extend $f_{i}$ along the fixed disk by the identity to the whole of $N$ and to $X=M \# N$ for a given oriented closed 4-manifold $M$. These extensions are also denoted by $f_{i}$. Obviously $f_{1}, \ldots, f_{n} \in \operatorname{Diff}^{+}(X)$ mutually commutes, and thus we get a homomorphism $\pi_{1}\left(T^{n}\right) \cong \mathbb{Z}^{n} \rightarrow \operatorname{Diff}^{+}(X)$. Using this action of $\pi_{1}\left(T^{n}\right)$ on $X$, we define a family on the torus $E \rightarrow T^{n}$ by $E:=$ $\mathbb{R}^{n} \times_{\pi_{1}\left(T^{n}\right)} X$. In other words, $E$ is the mapping torus of $f_{1}, \ldots, f_{n}$. We can now write down the following non-vanishing theorem:

Theorem 8.9. Let $M$ be an oriented closed smooth 4-manifold with $b^{+}(M) \geq 2$ and $\mathfrak{s}_{0}$ be the isomorphism class of a spin ${ }^{c}$ structure on $M$ such that the Seiberg-Witten invariant of $\left(M, \mathfrak{s}_{0}\right)$ is an odd number. Let $(X, \mathfrak{s})$ be the pair defined by

$$
(X, \mathfrak{s}):=\left(M \# n\left(S^{2} \times S^{2}\right), \mathfrak{s}_{0} \# \mathfrak{t}\right),
$$

where $\mathfrak{t}$ is the isomorphism class of a spin structure on $n\left(S^{2} \times S^{2}\right)$. Then, for the bundle $X \rightarrow E \rightarrow T^{n}$ constructed above, $\mathbb{S W}(E) \in H^{n}\left(T^{n} ; \mathbb{Z} / 2\right)$ is the generator of $H^{n}\left(T^{n} ; \mathbb{Z} / 2\right)$. In particular, we have

$$
\mathbb{S W}(X, \mathfrak{s}) \neq 0 \text { in } H^{n}(B \operatorname{Diff}(X, \mathfrak{s}) ; \mathbb{Z} / 2)
$$

by functoriality (Theorem [6.6).

Note that the condition $b^{+}(X) \geq n+2$ is obviously satisfied and it is easy to see that $d(\mathfrak{s})=-n$ in the situation of Theorem 8.9. We also note that, if $X$ is simply connected and $\mathfrak{s}$ is the isomorphism class of a $\operatorname{spin}^{c}$ structure coming from a spin structure on $X$, then we have $\operatorname{Diff}(X, \mathfrak{s})=\operatorname{Diff}^{+}(X)$. A spin structure on $X$ is unique up to isomorphism in this case.

Example 8.10. For $n>0$, let $\mathfrak{s}$ be the isomorphism class of a spin structure on $K 3 \# n\left(S^{2} \times S^{2}\right)$. Then we have

$$
\mathbb{S W}\left(K 3 \# n\left(S^{2} \times S^{2}\right), \mathfrak{s}\right) \neq 0 \text { in } H^{n}\left(B \operatorname{Diff}^{+}\left(K 3 \# n\left(S^{2} \times S^{2}\right)\right) ; \mathbb{Z} / 2\right) .
$$

Example 8.11. We here consider the connected sum of some copies of $S^{2} \times S^{2}$ as follows. (The author thanks David Baraglia for discussing this argument.) Using techniques given in J. Park [35], B. Hanke, D. Kotschick, and J. Wehrheim [15] have given oriented and closed 4-manifolds $X(p, q)$ involving two integer parameters $p, q$ (denoted by $k, n$ in [15] respectively) which are spin, symplectic, signature zero, with 
$b^{+} \geq 2$ and dissolve after connected summing one copy of $S^{2} \times S^{2}$ for sufficiently large $p, q$. Fix such $p, q$, and then we have $X(p, q) \# S^{2} \times S^{2} \cong m\left(S^{2} \times S^{2}\right)$ for some $m>0$. In Theorem 8.9, let us substitute $X(p, q)$ for $M$, and as $\mathfrak{s}_{0}$ take the (isomorphism class of a) $\operatorname{spin}^{c}$ structure coming from the symplectic structure of $X(p, q)$. Then, for a fixed $n>0$, we get

$$
\mathbb{S W}\left(k\left(S^{2} \times S^{2}\right), \mathfrak{s}\right) \neq 0 \text { in } H^{n}\left(B \operatorname{Diff}\left(k\left(S^{2} \times S^{2}\right), \mathfrak{s}\right) ; \mathbb{Z} / 2\right),
$$

where $k=m+n-1$. In addition, thanks to [15], one can find infinitely many such $k$ by varying $p, q$.

Remark 8.12. Since Hanke-Kotschick-Wehrheim [15] used Wall's theorem 42] to prove the above dissolving of $X(p, q) \# S^{2} \times S^{2}$, it seems difficult to determine $k$ 's in Example 8.11

The rest of this subsection is devoted to proving Theorem 8.9. One of the main tools of the proof is a technique due to D. Ruberman [38 40]: combination of wall-crossing and gluing. To use it, we describe wall-crossing on $N$. This is a spinanalogue of the argument of [18 by the author. Recall that the definition of the wall for the Seiberg-Witten equations. Let us fix a spin structure on $N$. In the space of perturbations

$$
\Pi(N)=\bigsqcup_{g \in \operatorname{Met}(N)} \Pi_{g}(N)=\bigsqcup_{g \in \operatorname{Met}(N)} L_{k-1}^{2}\left(\Lambda_{g}^{+}(N)\right),
$$

we have a codimenion- $n$ subspace

$$
\mathcal{W}(N):=\bigsqcup_{g \in \operatorname{Met}(N)} \mathcal{W}_{g}(N)
$$

where

$$
\mathcal{W}_{g}(N):=F_{A_{0}}^{+g}+\operatorname{Im} d^{+g}
$$

for a fixed reference connection $A_{0}$ of the determinant line bundle of the spin structure. We can take $A_{0}$ as a trivial connection, and then $\mathcal{W}_{g}(N)=\operatorname{Im} d^{+g}$. Set

$$
\stackrel{\circ}{\Pi}(N):=\Pi(N) \backslash \mathcal{W}(N), \quad \stackrel{\circ}{\Pi}_{g}(N):=\Pi_{g}(N) \backslash \mathcal{W}_{g}(N) .
$$

Then $\Pi(N)$ is homotopy equivalent to $S^{n-1}$. If a continuous map $\varphi:[0,1]^{n} \rightarrow \Pi(N)$ satisfying that $\varphi\left(\partial[0,1]^{n}\right) \subset \stackrel{\circ}{\Pi}(N)$ is given, we can therefore define the "intersection number" $\varphi \cdot \mathcal{W}(N)$ as the mapping degree of $\left.\varphi:\left([0,1]^{n}, \partial[0,1]^{n}\right)\right) \rightarrow(\Pi(N), \stackrel{\circ}{\Pi}(N))$. (Since we work on $\mathbb{Z} / 2$, we do not mention the convention on sign.) Denote by $\mathcal{H}_{g}^{+}(N)$ the space of self-dual harmonic 2-forms with respect to a metric $g$ on $N$. Let $g_{\bullet}:[0,1]^{n} \rightarrow \operatorname{Met}(N)$ be a family of metrics. For each element $\mathbf{t} \in[0,1]^{n}$, we write $g_{\mathbf{t}}$ for $g_{\bullet}(\mathbf{t})$. Let $V \subset H^{2}(N ; \mathbb{R})$ be a maximal positive definite subspace with respect to the intersection form and $\gamma_{1}, \ldots, \gamma_{n}$ be a basis of $V$. Let

$$
\text { Rest : } \bigsqcup_{\mathbf{t} \in[0,1]^{n}}\left(\Pi_{g_{\mathbf{t}}}(N), \stackrel{\circ}{\Pi}_{g_{\mathbf{t}}}(N)\right) \rightarrow \bigsqcup_{\mathbf{t} \in[0,1]^{n}}\left(\mathcal{H}_{g_{\mathbf{t}}}^{+}(N), \mathcal{H}_{g_{\mathbf{t}}}^{+}(N) \backslash\{0\}\right)
$$

be the restriction map, and define

$$
\Phi: \bigsqcup_{\mathbf{t} \in[0,1]^{n}}\left(\mathcal{H}_{g_{\mathbf{t}}}^{+}(N), \mathcal{H}_{g_{\mathbf{t}}}^{+}(N) \backslash\{0\}\right) \rightarrow\left(\mathbb{R}^{n}, \mathbb{R}^{n} \backslash\{0\}\right)
$$

by

$$
\Phi(\eta):=\left(\left\langle[\eta] \cup \gamma_{i},[N]\right\rangle\right)_{i=1}^{n}
$$


for $\eta \in \mathcal{H}_{g_{\mathrm{t}}}^{+}(N)$, and set $\Psi:=\Phi \circ$ Rest.

Lemma 8.13. The map $\Psi$ is a homotopy equivalent map between pairs.

Proof. Since $[0,1]^{n}$ is contractible, it suffices to see that the restriction of $\Psi$ to a

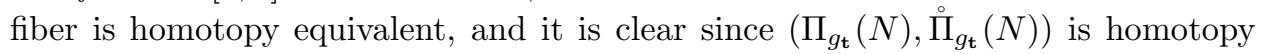
equivalent to $\left(\mathcal{H}_{g_{\mathbf{t}}}^{+}(N), \mathcal{H}_{g_{\mathbf{t}}}^{+}(N) \backslash\{0\}\right)$ and $\left.\Phi\right|_{\mathcal{H}_{g_{\mathbf{t}}}^{+}(N)}: \mathcal{H}_{g_{\mathbf{t}}}^{+}(N) \rightarrow \mathbb{R}^{n}$ is a linear isomorphism.

Corollary 8.14. Let $\sigma$ be a section

$$
\sigma:\left([0,1]^{n}, \partial[0,1]^{n}\right) \rightarrow \bigsqcup_{\mathbf{t} \in[0,1]^{n}}\left(\Pi_{g_{\mathbf{t}}}(N), \stackrel{\circ}{\Pi}_{g_{\mathbf{t}}}(N)\right)
$$

and define $\varphi:=\iota \circ \sigma:\left([0,1]^{n}, \partial[0,1]^{n}\right) \rightarrow(\Pi(N), \stackrel{\circ}{\Pi}(N))$, where

$$
\iota: \bigsqcup_{\mathbf{t} \in[0,1]^{n}}\left(\Pi_{g_{\mathbf{t}}}(N), \stackrel{\circ}{\Pi}_{g_{\mathbf{t}}}(N)\right) \rightarrow(\Pi(N), \stackrel{\circ}{\Pi}(N))
$$

is the inclusion. Then, $\varphi \cdot \mathcal{W}(N)$ is given as the mapping degree of the map

$$
\Psi \circ \sigma:\left([0,1]^{n}, \partial[0,1]^{n}\right) \rightarrow\left(\mathbb{R}^{n}, \mathbb{R}^{n} \backslash\{0\}\right) .
$$

We shall use Corollary 8.14 to prove Theorem 8.9. Before starting the proof of Theorem 8.9 , we give a map used in the proof. Let us write $[0,1]^{n}=I_{1} \times \cdots I_{n}$, where $I_{i}=[0,1]$ for each $i$. Decompose it as $I_{i}=I_{i, 1} \cup I_{i, 2}$, where $I_{i, 1}=[0,1 / 2]$ and $I_{i, 2}=[1 / 2,1]$. Then we obtain the decomposition

$$
[0,1]^{n}=\bigcup_{1 \leq a_{1}, \ldots, a_{n} \leq 2} I_{1, a_{1}} \times \cdots \times I_{n, a_{n}} .
$$

For each $i$, let $\Gamma_{i, 1}: I_{i, 1} \rightarrow I_{i, 1}$ be the identity map and $\Gamma_{i, 2}: I_{i, 2} \rightarrow I_{i, 1}$ be the projection onto $\{1 / 2\}$. Using these maps, we define a map

$$
\text { Shrink : }[0,1]^{n} \rightarrow I_{1,1} \times \cdots \times I_{n, 1}=[0,1 / 2]^{n}
$$

by

$$
\text { Shrink }:=\bigcup_{1 \leq a_{1}, \ldots, a_{n} \leq 2} \Gamma_{1, a_{1}} \times \cdots \times \Gamma_{n, a_{n}} .
$$

We next consider the following reparametrization map. Let us consider the homeomorphism $[0,1 / 2] \cong[0,1]$ given by an affine function. This map induces a homeomorphism Repa : $[0,1 / 2]^{n} \cong[0,1]^{n}$. Set the composition of these maps as

$$
F_{R S}:=\text { Repa oShrink }:[0,1]^{n} \rightarrow[0,1]^{n} \text {. }
$$

Intuitively, the pull-back by $F_{R S}$ is the following operation: for a given object on $[0,1]^{n}$, first give a copy of the object on $[0,1 / 2]^{n}$, and second extend it on the whole of $[0,1]^{n}$ by a natural pull-back.

Proof of Theorem 8.9. We first define $\gamma_{1}, \ldots, \gamma_{n}$ as follows. Let $\Sigma_{1, i}, \Sigma_{2, i} \subset N_{i}$ be the copies of $\Sigma_{1}$ and $\Sigma_{2}$ respectively. We define $\gamma_{i}:=$ P.D. $\left[\Sigma_{1, i}\right]+$ P.D. $\left[\Sigma_{2, i}\right]$ for each $i \in\{1, \ldots, n\}$. We next take $g$ • as follows. Let $g_{i}^{0}$ be a metric on $N_{i}$ which is a cylindrical near the boundary of an embedded disk in $N_{i}$. Take a path $h_{i}:[0,1] \rightarrow \operatorname{Met}\left(N_{i}\right)$ from $g_{i}^{0}$ to $f_{i}^{*} g_{i}^{0}$ and define $g_{\bullet}:[0,1]^{n} \rightarrow \operatorname{Met}(N)$ by

$$
g_{\mathbf{t}}=h_{1}\left(t_{1}\right) \# \cdots \# h\left(t_{n}\right)
$$


for $\mathbf{t}=\left(t_{1}, \ldots, t_{n}\right)$. Define faces $F_{i}^{0}$ and $F_{i}^{1}$ of $[0,1]^{n}$ by

$$
F_{i}^{0}:=\left\{\left(t_{1}, \ldots, t_{n}\right) \in[0,1]^{n} \mid t_{i}=0\right\}
$$

and

$$
F_{i}^{1}:=\left\{\left(t_{1}, \ldots, t_{n}\right) \in[0,1]^{n} \mid t_{i}=1\right\} .
$$

We define a map $\bar{\bullet}^{i}: F_{i}^{0} \rightarrow F_{i}^{1}$ by

$$
\mathbf{t}=\left(t_{1}, \ldots, 0, \ldots, t_{n}\right) \mapsto\left(t_{1}, \ldots, 1, \ldots, t_{n}\right)=\overline{\mathbf{t}}^{i}
$$

and similarly define $\overline{\boldsymbol{\bullet}}^{i}: F_{i}^{1} \rightarrow F_{i}^{0}$, which satisfies $\overline{\boldsymbol{\bullet}}^{i} \circ \overline{\boldsymbol{\bullet}}^{i}=\mathrm{id}$. Note that, for $\mathbf{t} \in F_{i}^{0}$, we have $f_{i}^{*} g_{\mathbf{t}}=g_{\overline{\mathbf{t}}^{i}}$. In what follows, we identify $H_{2}(N)$ with $H^{2}(N)$ by the Poincaré duality.

We next take a section $\sigma:\left([0,1]^{n}, \partial[0,1]^{n}\right) \rightarrow \bigsqcup_{\mathbf{t} \in[0,1]^{n}}\left(\Pi_{g_{\mathbf{t}}}(N),{\stackrel{\circ}{g_{\mathbf{t}}}}(N)\right)$ as follows. For each $i$, let $\hat{N}_{i}$ be the manifold obtained by gluing $N_{i} \backslash D^{4}$ with $S^{3} \times$ $[0, \infty)$, where $D^{4} \subset N_{i}$ is the disk used to consider the connected sum and $S^{3}$ is equipped with the standard metric. Note that a metric $h$ on $N_{i}$ induces $\hat{h} \in \operatorname{Met}\left(\hat{N}_{i}\right)$ if $h$ is a form of the product metric near $D^{4}$. For $t_{i} \in[0,1]$, let us consider

$$
D_{i}^{\hat{h}_{i}\left(t_{i}\right)}=d+\left(d^{+_{\hat{h}_{i}\left(t_{i}\right)}}\right)^{* \hat{h}_{i}\left(t_{i}\right)}: \Omega^{0}\left(\hat{N}_{i}\right) \oplus \Omega^{+\hat{h}_{i}\left(t_{i}\right)}\left(\hat{N}_{i}\right) \rightarrow \Omega^{1}\left(\hat{N}_{i}\right),
$$

which decomposes as $D_{i}^{\hat{h}_{i}\left(t_{i}\right)}=\partial / \partial t+L_{S^{3}}$ as in (33) on the cylindrical part. Let $\delta>0$ be a sufficiently small number such that $[-\delta, \delta] \backslash\{0\}$ contains no spectrum of $L_{S^{3}}$. Extend $D_{i}^{\hat{h}_{i}\left(t_{i}\right)}$ to a bounded Fredholm operator $D_{i}^{\hat{h}_{i}\left(t_{i}\right)}: L_{1, \delta}^{2}\left(\hat{N}_{i}\right) \rightarrow L_{\delta}^{2}\left(\hat{N}_{i}\right)$. As in Subsection 8.1, the notation $\operatorname{Ker} D_{i}^{\hat{h}_{i}\left(t_{i}\right)}$ denotes the kernel of this extended operator. As we mentioned in Subsection 8.1 Ker $D_{i}^{\hat{h}_{i}\left(t_{i}\right)}$ is isomorpshic to the space of extended $L^{2}$-solutions to $D_{i}^{\hat{h}_{i}\left(t_{i}\right)}$, which is nothing other than constant functions and extended self-dual $L^{2}$-harmonic forms on $\hat{N}_{i}$. Thus we have an embedding $\mathcal{H}_{L^{2}, \hat{h}_{i}\left(t_{i}\right)}^{+}\left(\hat{N}_{i}\right) \hookrightarrow \operatorname{Ker} D_{i}^{\hat{h}_{i}\left(t_{i}\right)}$, where $\mathcal{H}_{L^{2}, \hat{h}_{i}\left(t_{i}\right)}^{+}\left(\hat{N}_{i}\right)$ is the space of $\hat{h}_{i}\left(t_{i}\right)$-self-dual $L^{2}$-harmonic 2-forms on $\hat{N}_{i}$, which is of dimension $b^{+}\left(N_{i}\right)=1$. Take a section $\left\{\mu_{i}^{t_{i}}\right\}_{t_{i} \in[0,1]}$ of the bundle $\bigsqcup_{t_{i} \in[0,1]} \mathcal{H}_{L^{2}, \hat{h}_{i}\left(t_{i}\right)}^{+}\left(\hat{N}_{i}\right) \rightarrow[0,1]$ with $\left\|\mu_{i}^{t_{i}}\right\|_{L_{\delta}^{2}}=1$. There is no difficulty in extending the whole argument of Subsection 8.1 for connected sums of finitely many manifolds, not only for connected sums of two manifolds, and so we use it. Let $T \geq T_{1}, \ldots, T_{n}>0$ be positive numbers satisfying that $4 T \geq 3\left(T_{i}+T_{j}\right)$ for any $i, j \in\{1, \ldots, n\}$ with $i \neq j$ and that $T_{i}>C_{1}$ for any $i$, where $C_{1}$ is the constant given in Subsection 8.1. We take $T \geq T_{1}, \ldots, T_{n}$ to be sufficiently large, which shall be described below. In the definition for the connected sum $N=\#_{i=1}^{n} N_{i}$, we use the cylinders of length $2 T$ as in Subsection 8.1 . For $\mathbf{t} \in[0,1]^{n}$, let us consider

$$
D=D^{g_{\mathbf{t}}}=d+\left(d^{+g_{\mathbf{t}}}\right)^{* g_{\mathbf{t}}}: \Omega^{0}(N) \oplus \Omega^{+g_{\mathbf{t}}}(N) \rightarrow \Omega^{1}(N) .
$$

Let $\check{\mu}_{i}^{\mathbf{t}} \in \operatorname{Ker} D^{g_{\mathbf{t}}}$ be the almost localized self-dual harmonic form on $N_{i}$ arising from $\mu_{i}^{t_{i}}$ with respect to $T, T_{1}, \ldots, T_{n}$ and $\hat{h}_{1}\left(t_{1}\right), \ldots, \hat{h}_{n}\left(t_{n}\right)$. Define a section

$$
\alpha_{i}:[0,1]^{n} \rightarrow \bigsqcup_{\mathbf{t} \in[0,1]^{n}} \Pi_{g_{\mathbf{t}}}(N)
$$

by $\alpha_{i}(\mathbf{t}):=\check{\mu}_{i}^{\mathbf{t}}$ for each $i$. Using the map (36), let us consider

$$
\tilde{g}_{\bullet}:=F_{R S}^{*} g_{\bullet}:[0,1]^{n} \rightarrow \operatorname{Met}(N) \text {. }
$$


We again write $\tilde{g}_{\mathbf{t}}$ for $\tilde{g}_{\bullet}(\mathbf{t})$. Similarly, let us consider the pull-backed section

$$
\tilde{\alpha}_{i}:=F_{R S}^{*} \alpha_{i}:[0,1]^{n} \rightarrow \bigsqcup_{\mathbf{t} \in[0,1]^{n}} \Pi_{\tilde{g}_{\mathbf{t}}}(N) .
$$

By definition we have $\tilde{g}_{\mathbf{t}}=g_{\mathbf{s}}$ for $\mathbf{s}=F_{R S}(\mathbf{t})$ and $\tilde{\alpha}_{i}(\mathbf{t})=\check{\mu}_{i}^{\mathbf{s}}$. We now define a continuous section

$$
\beta_{i}:[0,1]^{n} \rightarrow \bigsqcup_{\mathbf{t} \in[0,1]^{n}} \mathcal{H}_{\tilde{g}_{\mathbf{t}}}^{+}(N)
$$

as follows. For $\mathbf{t}=\left(t_{1}, \ldots, t_{n}\right)$, if $0 \leq t_{i} \leq 1 / 2$ holds, we define $\beta_{i}(\mathbf{t}):=\tilde{\alpha}_{i}(\mathbf{t})$, and if $1 / 2 \leq t_{i} \leq 1$ holds, we define

$$
\beta_{i}(\mathbf{t}):=\left(1-t_{i}\right) \tilde{\alpha}_{i}(\mathbf{t})+t_{i} f_{i}^{*} \check{\mu}_{i}^{\overline{\mathbf{s}}^{i}}
$$

for $\mathbf{s}=F_{R S}(\mathbf{t})$. We here check that, if $\mathbf{t}$ satisfies that $1 / 2 \leq t_{i} \leq 1$, then $f_{i}^{*} \breve{\mu}_{i}^{i} \in$ $\mathcal{H}_{\tilde{g}_{\mathrm{t}}}^{+}(N)$ holds. If $1 / 2 \leq t_{i} \leq 1$ holds, we have $\overline{\mathbf{s}}^{i} \in F_{i}^{0}$, and therefore it follows that $f_{i}^{*} g_{\overline{\mathbf{s}}^{i}}=g_{\mathbf{s}}=\tilde{g}_{\mathbf{t}}$. This gives the map $f_{i}^{*}: \mathcal{H}_{g_{\overline{\mathbf{s}}}}^{+}(N) \rightarrow \mathcal{H}_{\tilde{g}_{\mathbf{t}}}^{+}(N)$, thus we get $f_{i}^{*} \check{\mu}_{i}^{\mathbf{s}^{i}} \in$ $\mathcal{H}_{\tilde{g}_{\mathbf{t}}}^{+}(N)$. We define a section $\sigma:\left([0,1]^{n}, \partial[0,1]^{n}\right) \rightarrow \bigsqcup_{\mathbf{t} \in[0,1]^{n}}\left(\Pi_{g_{\mathbf{t}}}(N),{\stackrel{\circ}{g_{\mathbf{t}}}}(N)\right)$ by

$$
\sigma(\mathbf{t}):=\beta_{1}(\mathbf{t})+\cdots+\beta_{n}(\mathbf{t}) .
$$

Obviously $\sigma$ factors through the inclusion

$$
\bigsqcup_{\mathbf{t} \in[0,1]^{n}}\left(\mathcal{H}_{g_{\mathbf{t}}}^{+}(N), \mathcal{H}_{g_{\mathbf{t}}}^{+}(N) \backslash\{0\}\right) \hookrightarrow \bigsqcup_{\mathbf{t} \in[0,1]^{n}}\left(\Pi_{g_{\mathbf{t}}}(N), \stackrel{\circ}{\Pi}_{g_{\mathbf{t}}}(N)\right) .
$$

For this $\sigma$, we shall show that the mapping degree of the map

$$
\Psi \circ \sigma:\left([0,1]^{n}, \partial[0,1]^{n}\right) \rightarrow\left(\mathbb{R}^{n}, \mathbb{R}^{n} \backslash\{0\}\right) .
$$

is 1 , up to sign. Here $\Psi$ is the map given before Lemma 8.13 If we assume this calculation of the mapping degree, the rest story is similar to Subsection 3.2 of [20, given as follows. Let us consider a cell structure of $[0,1]^{n}$ admitting a unique $n$-cell. We equip $T^{n}$ with the cell structure obtained from the given cell structure of $[0,1]^{n}$ by identifying $F_{i}^{0}$ with $F_{i}^{1}$ for each $i$. We now use D. Ruberman's combination of wall-crossing and gluing arguments [38 40]. (It is summarized as Proposition 4.1 in [19.) Take a generic element $\mu_{M} \in \Pi(M)$ and define $\tilde{\sigma}:=\mu_{M} \# \sigma:[0,1]^{n} \rightarrow \bigsqcup_{\mathbf{t} \in[0,1]^{n}} \Pi_{\pi\left(\mu_{M}\right) \# g_{\mathbf{t}}}(X)$, where $\pi: \Pi(M) \rightarrow \operatorname{Met}(M)$ is the projection. Ruberman's result implies that the counted number of the parameterized moduli space with respect to $\tilde{\sigma}$ coincides with the product of $\varphi \cdot \mathcal{W}(N)$ and the Seiberg-Witten invariant of $\left(M, \mathfrak{s}_{0}\right)$ under a small perturbation, and it is non-zero over $\mathbb{Z} / 2$ because of Corollary 8.14 and our assumption on $\left(M, \mathfrak{s}_{0}\right)$. Since $\sigma$ satisfies that $f_{i}^{*} \sigma(\mathbf{t})=\sigma\left(\overline{\mathbf{t}}^{i}\right)$ for each $i$ and $\mathbf{t} \in F_{i}^{0}, \tilde{\sigma}$ can be regarded as an inductive section $\tilde{\sigma}: T^{n} \rightarrow \Pi(E)$ for the bundle $E$, and the moduli space parameterized on $[0,1]^{n}$ is isomorphic to the moduli space $\mathcal{M}_{\tilde{\sigma}}$ parameterized on $T^{n}$. Here we used the vanishing of the moduli space on $\partial[0,1]^{n}$. Thus we obtain the non-triviality of the counted number of $\mathcal{M}_{\tilde{\sigma}}$ over $\mathbb{Z} / 2$, which is nothing other than $\mathfrak{m}\left(s_{\varphi_{e}^{*} \tilde{\sigma}}, 1 ; D_{e}^{n}\right)=1$ in $\mathbb{Z} / 2$. Here $e$ is the unique $n$-cell of $T^{n}$ with respect to the given cell structure and $\varphi_{e}$ is the characteristic map of $e$. Hence $\mathbb{S W}(E)$ is the generator of $H^{n}\left(T^{n} ; \mathbb{Z} / 2\right)$.

Our remaining task is to show that the mapping degree of $\Psi \circ \sigma$ is \pm 1 . To do it, for $i, j \in\{1, \ldots, n\}$ and $\mathbf{t}=\left(t_{1}, \ldots, t_{n}\right)$, we need to investigate $\left\langle\beta_{j}(\mathbf{t}) \cup \gamma_{i},[N]\right\rangle$. We divide our consideration into the four cases: Case 1-1, Case 1-2, Case 2-1, and Case 2-2. They correspond to whether $t_{j} \leq 1 / 2$ or not and whether $i=j$ 
or not. For $\mathbf{s}=F_{R S}(\mathbf{t})$, we write $\mathbf{s}=\left(s_{1}, \ldots, s_{n}\right), \overline{\mathbf{s}}^{i}=\left(\bar{s}_{1}, \ldots, \bar{s}_{n}\right)$ and set $\vec{\mu}_{j}^{s_{j}}=\left(0, \ldots, \mu_{j}^{s_{j}}, \ldots, 0\right), \vec{\mu}_{i}^{\bar{s}_{j}}=\left(0, \ldots, \mu_{j}^{\bar{s}_{j}}, \ldots, 0\right)$, where $\mu_{j}^{s_{j}}$ and $\mu_{j}^{\bar{s}_{j}}$ are in the $j$-th components.

Case 1-1: Assume that $0 \leq t_{j} \leq 1 / 2$ and $i=j$. We first have

$$
\begin{aligned}
\left\langle\beta_{i}(\mathbf{t}) \cup \gamma_{i},[N]\right\rangle= & \int_{\Sigma_{1, i}} \check{\mu}_{i}^{\mathbf{s}}+\int_{\Sigma_{2, i}} \check{\mu}_{i}^{\mathbf{s}} \\
= & \int_{\Sigma_{1, i}} \theta_{i, T_{i}}\left(\vec{\mu}_{i}^{s_{i}}\right)+\int_{\Sigma_{2, i}} \theta_{i, T_{i}}\left(\vec{\mu}_{i}^{s_{i}}\right) \\
& +\int_{\Sigma_{1, i}} Q_{i, T_{i}}^{X} D \theta_{i, T_{i}}\left(\vec{\mu}_{i}^{s_{i}}\right)+\int_{\Sigma_{2, i}} Q_{i, T_{i}}^{X} D \theta_{i, T_{i}}\left(\vec{\mu}_{i}^{s_{i}}\right)
\end{aligned}
$$

For the first two terms of the right-hand side of (37), note that

$$
\int_{\Sigma_{1, i}} \theta_{i, T_{i}}\left(\vec{\mu}_{i}^{s_{i}}\right)+\int_{\Sigma_{2, i}} \theta_{i, T_{i}}\left(\vec{\mu}_{i}^{s_{i}}\right)=\int_{\Sigma_{1, i}} \mu_{i}^{s_{i}}+\int_{\Sigma_{2, i}} \mu_{i}^{s_{i}} .
$$

We next consider the last two terms of the right-hand side of (37). Let us take a metric $g^{\prime}$ on $N$ such that a neighborhood of $\Sigma_{k, i}$ is isometric to $D^{2} \times \Sigma_{k, i}$ equipped with the product metric for $k=1,2$. Here $\Sigma_{k, i}$ is regarded as $\{0\} \times \Sigma_{k, i}$. Let $u$ be a coordinate of $D^{2}$. Near a neighborhood of $\Sigma_{1, i}$, we have $Q_{i, T_{i}}^{X} D \theta_{i, T_{i}}\left(\vec{\mu}_{i}^{s_{i}}\right)=\mu_{i}^{s_{i}}-\check{\mu}_{i}^{\mathbf{s}}$ and the right-hand side is a closed form, and so is the left-hand side. It therefore follows that

$$
\int_{\{u\} \times \Sigma_{k, i}} Q_{i, T_{i}}^{X} D \theta_{i, T_{i}}\left(\vec{\mu}_{i}^{s_{i}}\right)=\int_{\Sigma_{k, i}} Q_{i, T_{i}}^{X} D \theta_{i, T_{i}}\left(\vec{\mu}_{i}^{s_{i}}\right)
$$

for any $u \in D^{2}$. Thus we have

$$
\begin{aligned}
\left|\int_{\Sigma_{k, i}} Q_{i, T_{i}}^{X} D \theta_{i, T_{i}}\left(\vec{\mu}_{i}^{s_{i}}\right)\right|^{2} & =\left|\int_{\{u\} \times \Sigma_{k, i}} Q_{i, T_{i}}^{X} D \theta_{i, T_{i}}\left(\vec{\mu}_{i}^{s_{i}}\right)\right|^{2} \\
& \leq \operatorname{Area}\left(\Sigma_{k, i}\right) \cdot \int_{\{u\} \times \Sigma_{k, i}}\left|Q_{i, T_{i}}^{X} D \theta_{i, T_{i}}\left(\vec{\mu}_{i}^{s_{i}}\right)\right|^{2} .
\end{aligned}
$$

On the other hand, Fubini's theorem implies that

$$
\begin{aligned}
\int_{D^{2}} \int_{\{u\} \times \Sigma_{k, i}}\left|Q_{i, T_{i}}^{X} D \theta_{i, T_{i}}\left(\vec{\mu}_{i}^{s_{i}}\right)\right|^{2} & =\int_{D^{2} \times \Sigma_{k, i}}\left|Q_{i, T_{i}}^{X} D \theta_{i, T_{i}}\left(\vec{\mu}_{i}^{s_{i}}\right)\right|^{2} \\
& \leq\left\|Q_{i, T_{i}}^{X} D \theta_{i, T_{i}}\left(\vec{\mu}_{i}^{s_{i}}\right)\right\|_{L^{2}\left(N, g^{\prime}\right)}^{2} \\
& \leq C_{4}\left\|Q_{i, T_{i}}^{X} D \theta_{i, T_{i}}\left(\vec{\mu}_{i}^{s_{i}}\right)\right\|_{L^{2}\left(N, g_{\mathbf{s}}\right)}^{2} .
\end{aligned}
$$

Here $C_{\bullet}$ denotes a constant which is independent of $T, T_{1}, \ldots, T_{n}$. From this inequality, the inequality (39), and Lemma 8.2, we have

$$
\begin{aligned}
\left|\int_{\Sigma_{k, i}} Q_{i, T_{i}}^{X} D \theta_{i, T_{i}}\left(\vec{\mu}_{i}^{s_{i}}\right)\right|^{2} & \leq \frac{\operatorname{Area}\left(\Sigma_{k, i}\right)}{\operatorname{Area}\left(D^{2}\right)} \int_{D^{2}} \int_{\{u\} \times \Sigma_{k, i}}\left|Q_{i, T_{i}}^{X} D \theta_{i, T_{i}}\left(\vec{\mu}_{i}^{s_{i}}\right)\right|^{2} \\
& \leq C_{5}\left\|Q_{i, T_{i}}^{X} D \theta_{i, T_{i}}\left(\vec{\mu}_{i}^{s_{i}}\right)\right\|_{L^{2}\left(N, g_{\mathbf{t}}\right)}^{2} \leq \frac{C_{6}}{T_{i}^{2}}
\end{aligned}
$$

This inequality and the equalities (37), (38) imply that

$$
\left|\left\langle\beta_{i}(\mathbf{t}) \cup \gamma_{i},[N]\right\rangle-\left(\int_{\Sigma_{1, i}} \mu_{i}^{s_{i}}+\int_{\Sigma_{2, i}} \mu_{i}^{s_{i}}\right)\right| \leq \frac{C_{7}}{T_{i}} .
$$


Case 1-2: Assume that $0 \leq t_{j} \leq 1 / 2$ and $i \neq j$. Then we have

$$
\int_{\Sigma_{1, i}} \theta_{j, T_{j}}\left(\vec{\mu}_{j}^{s_{j}}\right)+\int_{\Sigma_{2, i}} \theta_{j, T_{j}}\left(\vec{\mu}_{j}^{s_{j}}\right)=0
$$

In addition, near a neighborhood of $\Sigma_{k, i}(k=1,2)$, we get $Q_{j, T_{j}}^{X} D \theta_{j, T_{j}}\left(\vec{\mu}_{j}^{s_{j}}\right)=-\check{\mu}_{j}^{\mathbf{s}}$, which is a closed form. An argument which is similar to Case 1-1 therefore works, and thus we obtain

$$
\left|\left\langle\beta_{j}(\mathbf{t}) \cup \gamma_{i},[N]\right\rangle\right| \leq \frac{C_{8}}{T_{j}}
$$

Case 2-1: Assume that $1 / 2<t_{j} \leq 1$ and $i=j$. We first have

$$
\left\langle\beta_{i}(\mathbf{t}) \cup \gamma_{i},[N]\right\rangle=\left(1-t_{i}\right)\left(\int_{\Sigma_{1, i}} \check{\mu}_{i}^{\mathbf{s}}+\int_{\Sigma_{2, i}} \check{\mu}_{i}^{\mathbf{s}}\right)+t_{i}\left(\int_{\Sigma_{1, i}} f_{i}^{*} \check{\mu}_{i}^{\overline{\mathbf{s}}^{i}}+\int_{\Sigma_{2, i}} f_{i}^{*} \check{\mu}_{i}^{\overline{\mathbf{s}}^{i}}\right)
$$

For the first term of the right-hand side of this equation, we have shown (40) in Case 1-1, and therefore consider the second term. We have

$$
\begin{aligned}
\int_{\Sigma_{1, i}} f_{i}^{*} \check{\mu}_{i}^{i}+\int_{\Sigma_{2, i}} f_{i}^{*} \check{\mu}_{i}^{i}= & \int_{\Sigma_{1, i}} f_{i}^{*} \theta_{i, T_{i}}\left(\vec{\mu}_{i}^{\bar{s}_{i}}\right)+\int_{\Sigma_{2, i}} f_{i}^{*} \theta_{i, T_{i}}\left(\vec{\mu}_{i}^{\bar{s}_{i}}\right) \\
& +\int_{\Sigma_{1, i}} Q_{i, T_{i}}^{X} D \theta_{i, T_{i}}\left(\vec{\mu}_{i}^{\bar{s}_{i}}\right)+\int_{\Sigma_{2, i}} Q_{i, T_{i}}^{X} D \theta_{i, T_{i}}\left(\vec{\mu}_{i}^{\bar{s}_{i}}\right)
\end{aligned}
$$

and

$$
\int_{\Sigma_{1, i}} f_{i}^{*} \theta_{i, T_{i}}\left(\vec{\mu}_{i}^{\bar{s}_{i}}\right)+\int_{\Sigma_{2, i}} f_{i}^{*} \theta_{i, T_{i}}\left(\vec{\mu}_{i}^{\bar{s}_{i}}\right)=\int_{\Sigma_{1, i}} f_{i}^{*} \mu_{i}^{\bar{s}_{i}}+\int_{\Sigma_{2, i}} f_{i}^{*} \mu_{i}^{\bar{s}_{i}}
$$

Let us consider the last two terms of the right-hand side of (43). For $k=1$, 2, we have $Q_{i, T_{i}}^{X} D \theta_{i, T_{i}}\left(\vec{\mu}_{i}^{\bar{s}_{i}}\right)=\mu_{i}^{\bar{s}_{i}}-\check{\mu}_{i}^{\overline{\mathbf{s}}}$ near $f_{i}\left(\Sigma_{k, i}\right)$, and hence $f_{i}^{*} Q_{i, T_{i}}^{X} D \theta_{i, T_{i}}\left(\vec{\mu}_{i}^{\bar{s}_{i}}\right)$ is a closed from near $\Sigma_{k, i}$. Therefore, as in Case 1-1, we have

$$
\begin{aligned}
\left|\int_{\Sigma_{k, i}} f_{i}^{*} Q_{i, T_{i}}^{X} D \theta_{i, T_{i}}\left(\vec{\mu}_{i}^{\bar{s}_{i}}\right)\right|^{2} & \leq C_{9} \int_{D^{2} \times \Sigma_{k, i}}\left|f_{i}^{*} Q_{i, T_{i}}^{X} D \theta_{i, T_{i}}\left(\vec{\mu}_{i}^{\bar{s}_{i}}\right)\right|^{2} \\
& \leq C_{9} \int_{N_{i} \backslash D^{4}}\left|f_{i}^{*} Q_{i, T_{i}}^{X} D \theta_{i, T_{i}}\left(\vec{\mu}_{i}^{\bar{s}_{i}}\right)\right|^{2} \\
& \leq C_{9}\left\|f_{i}^{*}\right\|_{\mathrm{op}}^{2} \int_{N_{i} \backslash D^{4}}\left|Q_{i, T_{i}}^{X} D \theta_{i, T_{i}}\left(\vec{\mu}_{i}^{\bar{s}_{i}}\right)\right|^{2} \\
& \leq C_{10}\left\|Q_{i, T_{i}}^{X} D \theta_{i, T_{i}}\left(\vec{\mu}_{i}^{\bar{s}_{i}}\right)\right\|_{L^{2}\left(N, g^{\prime}\right)}^{2} \\
& \leq C_{11}\left\|Q_{i, T_{i}}^{X} D \theta_{i, T_{i}}\left(\vec{\mu}_{i}^{\bar{s}_{i}}\right)\right\|_{L^{2}\left(N, \bar{g}_{\mathrm{s}}\right)}^{2} \leq \frac{C_{12}}{T_{i}^{2}} .
\end{aligned}
$$

Here we used the decomposition $N=\left(N_{i} \backslash D^{4}\right) \sqcup\left(\#_{j \neq i} N_{j}\right) \backslash D^{4}$ taken so that the all cylinders of length $2 T$ are contained in $\left(\#_{j \neq i} N_{j}\right) \backslash D^{4}$, and $\left\|f_{i}^{*}\right\|_{\text {op }}$ is the operator norm for the operator acting on $L^{2}\left(N_{i} \backslash D^{4}\right)$. Since $N_{i} \backslash D^{4}$ contains no cylindrical part, $\left\|f_{i}^{*}\right\|_{\text {op }}$ is independent of $T, T_{1}, \ldots, T_{n}$, and so it can be absorbed into $C_{10}$. 
HOKUTO KONNO

From the equalities (42), (43), (44) and the inequalities (40), (45), it follows that (46)

$$
\begin{aligned}
& \left|\left\langle\beta_{i}(\mathbf{t}) \cup \gamma_{i},[N]\right\rangle-\left(1-t_{i}\right)\left(\int_{\Sigma_{1, i}} \mu_{i}^{s_{i}}+\int_{\Sigma_{2, i}} \mu_{i}^{s_{i}}\right)-t_{i}\left(\int_{\Sigma_{1, i}} f_{i}^{*} \mu_{i}^{\bar{s}_{i}}+\int_{\Sigma_{2, i}} f_{i}^{*} \mu_{i}^{\bar{s}_{i}}\right)\right| \\
& \leq \frac{C_{7}}{T_{i}}+\frac{\sqrt{C_{12}}}{T_{i}}=\frac{C_{13}}{T_{i}} .
\end{aligned}
$$

Case 2-2: Assume that $1 / 2<t_{j} \leq 1$ and $i \neq j$. We first have

$$
\left\langle\beta_{i}(\mathbf{t}) \cup \gamma_{i},[N]\right\rangle=\left(1-t_{j}\right)\left(\int_{\Sigma_{1, i}} \check{\mu}_{j}^{\mathbf{s}}+\int_{\Sigma_{2, i}} \check{\mu}_{j}^{\mathbf{s}}\right)+t_{j}\left(\int_{\Sigma_{1, i}} f_{j}^{*} \check{\mu}_{j}^{\overline{\mathbf{s}}^{j}}+\int_{\Sigma_{2, i}} f_{j}^{*} \check{\mu}_{j}^{\mathbf{s}^{j}}\right) .
$$

For the first term of the right-hand side of this equation, as Case 1-2, we obtain

$$
\left|\int_{\Sigma_{1, i}} \check{\mu}_{j}^{\mathbf{s}}+\int_{\Sigma_{2, i}} \check{\mu}_{j}^{\mathbf{s}}\right| \leq \frac{C_{14}}{T_{j}} .
$$

For the second term, note that

$$
\int_{\Sigma_{k, i}} f_{j}^{*} \check{\mu}_{j}^{\overline{\mathbf{s}}^{j}}=\int_{\Sigma_{k, i}} \check{\mu}_{j}^{\overline{\mathbf{s}}^{j}}
$$

holds for $k=1,2$. Therefore, as Case 1-2 again, we obtain

$$
\left|\int_{\Sigma_{1, i}} f_{j}^{*} \breve{\mu}_{j}^{\overline{\mathbf{s}}^{j}}+\int_{\Sigma_{2, i}} f_{j}^{*} \breve{\mu}_{j}^{\overline{\mathbf{s}}^{j}}\right| \leq \frac{C_{15}}{T_{j}} .
$$

From the equality (47) and the inequalities (48), (150), it follows that

$$
\left|\left\langle\beta_{j}(\mathbf{t}) \cup \gamma_{i},[N]\right\rangle\right| \leq \frac{C_{16}}{T_{j}} .
$$

We have now completed investigating all four cases. For $i \in\{1, \ldots, n\}$ and $\mathbf{t} \in F_{i}^{0}$, using (40) in Case 1-1, (41) in Case 1-2, and (50) in Case 2-2, we have

$$
\begin{aligned}
& \left|\left\langle\sigma(\mathbf{t}) \cup \gamma_{i},[N]\right\rangle-\left(\int_{\Sigma_{1, i}} \mu_{i}^{0}+\int_{\Sigma_{2, i}} \mu_{i}^{0}\right)\right| \\
\leq & \left|\left\langle\beta_{i}(\mathbf{t}) \cup \gamma_{i},[N]\right\rangle-\left(\int_{\Sigma_{1, i}} \mu_{i}^{0}+\int_{\Sigma_{2, i}} \mu_{i}^{0}\right)\right|+\sum_{j \neq i}\left|\left\langle\beta_{j}(\mathbf{t}) \cup \gamma_{i},[N]\right\rangle\right| \\
\leq & \frac{C_{7}}{T_{i}}+\sum_{\substack{j \neq i \\
0 \leq t_{j} \leq 1 / 2}} \frac{C_{8}}{T_{j}}+\sum_{\substack{j \neq i \\
1 / 2<t_{j} \leq 1}} \frac{C_{16}}{T_{j}} \leq \frac{C_{17}}{\min \left\{T_{j} \mid 1 \leq j \leq n\right\}} .
\end{aligned}
$$

Similarly, for $\mathbf{t} \in F_{i}^{1}$, using (46) in Case 2-1, and (41) in Case 1-2, (50) in Case 2-2, we have

$$
\begin{aligned}
& \left|\left\langle\sigma(\mathbf{t}) \cup \gamma_{i},[N]\right\rangle-\left(\int_{\Sigma_{1, i}} f_{i}^{*} \mu_{i}^{0}+\int_{\Sigma_{2, i}} f_{i}^{*} \mu_{i}^{0}\right)\right| \\
\leq & \frac{C_{13}}{T_{i}}+\sum_{\substack{j \neq i \\
0 \leq t_{j} \leq 1 / 2}} \frac{C_{8}}{T_{j}}+\sum_{\substack{j \neq i \\
1 / 2<t_{j} \leq 1}} \frac{C_{16}}{T_{j}} \leq \frac{C_{18}}{\min \left\{T_{j} \mid 1 \leq j \leq n\right\}} .
\end{aligned}
$$


We can regard $\gamma_{i}$ as a cohomology class on $\hat{N}_{i}$. Since $f_{i}^{*} \gamma_{i}=-\gamma_{i}$ holds, we have

$$
\int_{\Sigma_{1, i}} f_{i}^{*} \mu_{i}^{0}+\int_{\Sigma_{2, i}} f_{i}^{*} \mu_{i}^{0}=\int_{\hat{N}_{i}} f_{i}^{*}\left[\mu_{i}^{0}\right] \cup \gamma_{i}=-\int_{\hat{N}_{i}}\left[\mu_{i}^{0}\right] \cup \gamma_{i}=-\left(\int_{\Sigma_{1, i}} \mu_{i}^{0}+\int_{\Sigma_{2, i}} \mu_{i}^{0}\right)
$$

Since $\operatorname{Ker} D_{i}^{\hat{h}_{i}(0)} \cap \Omega^{2}(N)$ is isomorphic to $H^{+}\left(\hat{N}_{i}\right)$ via Hodge theory, $\left[\mu_{i}^{0}\right]$ is of positive self-intersection, and hence the quantity in (53) is non-zero. Therefore, if we take $T, T_{1}, \ldots, T_{n}$ to be sufficiently large, the inequalities (51), (152) and the equality (53) imply that $\left\langle\sigma(\mathbf{t}) \cup \gamma_{i},[N]\right\rangle$ for $\mathbf{t} \in F_{i}^{0}$ and that for $\mathbf{t} \in F_{i}^{1}$ have different signs. This means that $\Psi \circ \sigma\left(F_{i}^{0}\right)$ and $\Psi \circ \sigma\left(F_{i}^{1}\right)$ are contained in distinct connected componect of $\mathbb{R}^{n} \backslash(\mathbb{R} \times \cdots \times\{0\} \times \cdots \times \mathbb{R})$, where $\{0\}$ is the $i$-th component. This implies that the mapping degree of $\left.\Psi \circ \sigma\right|_{\partial[0,1]^{n}}: \partial[0,1]^{n} \rightarrow \mathbb{R}^{n} \backslash\{0\}$ is 1 up to sign, and this coincides with that of $\Psi \circ \sigma:\left([0,1]^{n}, \partial[0,1]^{n}\right) \rightarrow\left(\mathbb{R}^{n}, \mathbb{R}^{n} \backslash\{0\}\right)$. This completes the proof of Theorem 8.9 .

Remark 8.15. Since the diffeomorphisms $f_{i}$ used in Theorem 8.9 reverse a given homology orientation $\mathcal{O}$ of $X$, we cannot give a non-trivial element of $H^{*}(B \operatorname{Diff}(X, \mathfrak{s}, \mathcal{O}) ; \mathbb{Z})$ using these diffeomorphisms. At this stage the author does not know how to give a cohomology class of higher degree over $\mathbb{Z}$ using $\mathbb{S W}$. On the other hand, thanks to D. Ruberman's example in [38, we can give a non-trivial element of $H^{1}(B \operatorname{Diff}(X, \mathfrak{P}, \mathcal{O}) ; \mathbb{Z})$ for a suitable $(X, \mathfrak{P})$ using $\mathbb{D}$, described in Subsection 8.5 ,

8.3. Behavior under the composition 1. To give subtle examples of calculations for $\mathbb{S W}$, we combine an argument of $[20$ by the author and one of $\mathrm{D}$. Ruberman 38. Ruberman has defined invariants of diffeomorphisms on an oriented closed 4-manifold $X$ in 38 using 1-parameter families of $S O(3)$-ASD equations and Seiberg-Witten equations. In 38 he also has given examples of diffeomorphisms on some 4-manifold which are topologically isotopic to the identity map, but not smoothly isotopic to it. To prove the former property, he considered the composition of two diffeomorphisms having mutually converse actions on the homology groups, and to prove the latter property, he used the invariant based on $S O(3)$ Yang-Mills ASD equations. His family corresponding to a given diffeomorphism can be regarded as a family of ASD equations parameterized on $S^{1}$ via the mapping torus construction for the diffeomorphism. In this subsection we consider a Seiberg-Witten version and higher-dimensional parameter version of Ruberman's argument. We give a bundle of a 4 -manifold $X$ obtained as the mapping torus of a tuple of commutative diffeomorphisms belonging to $\operatorname{Diff}(X, \mathfrak{s})$ for (the isomorphism class of) a $\operatorname{spin}^{c}$ structure $\mathfrak{s}$ on $X$ such that each diffeomorphism is topologically isotopic to the identity, but the bundle is non-trivial as $\operatorname{Diff}(X, \mathfrak{s})$-bundle. In this subsection we consider an example of a bundle with non-spin fiber, and we shall do one with spin fiber in Subsection 8.4 using an argument of Subsection 8.2

Fix $n>0$ and let $M_{0}$ be an oriented closed smooth 4-manifold with $b^{+}\left(M_{0}\right) \geq 2$ and $\mathfrak{s}_{0}$ be the isomorpshim class of a $\operatorname{spin}^{c}$ structure on $M_{0}$. Assume that the Seiberg-Witten invariant of $\left(M, \mathfrak{s}_{0}\right)$ is an odd number and that $M_{0} \# \mathbb{C P}^{2}$ is diffeomorphic to $M_{1} \# \mathbb{C P}^{2}$, where $M_{1}=b^{+}(M) \mathbb{C P}^{2} \# b^{-}(M)\left(-\mathbb{C P}^{2}\right.$ ). (Typically we can take a simply connected elliptic surface and the complex structure of it as $\left(M_{0}, \mathfrak{s}_{0}\right)$.) Let us denote $H_{i}$ the projective line embedded in the right connected summand of $M_{i} \# \mathbb{C P}^{2}$. As noted in Ruberman [38, we can find a diffeomorphism $\varphi: M_{0} \# \mathbb{C P}^{2} \rightarrow M_{1} \# \mathbb{C P}^{2}$ so that $\varphi\left(H_{1}\right)$ is homologous to $H_{2}$. The induced map 
$\varphi^{*}: H^{2}\left(M_{1} \# \mathbb{C P}^{2}\right) \rightarrow H^{2}\left(M_{0} \# \mathbb{C P}^{2}\right)$ acts as identity on $H^{2}\left(\mathbb{C P}^{2}\right)$. We can therefore define the isomorphism class $\mathfrak{s}_{1}$ of a $\operatorname{spin}^{c}$ structure on $M_{1}$ by $\mathfrak{s}_{1}:=\left(\varphi^{-1}\right)^{*} \mathfrak{s}_{0}$. Extending by the identity, we get a diffeomorphism $\varphi: M_{0} \# \mathbb{C P}^{2} \# 2\left(-\mathbb{C P}^{2}\right) \rightarrow$ $M_{1} \# \mathbb{C P}^{2} \# 2\left(-\mathbb{C P}^{2}\right)$. Let $\mathfrak{t}_{0}$ be the isomorphism class of a $\operatorname{spin}^{c}$ structure on $\mathbb{C P}^{2} \# 2\left(-\mathbb{C P}^{2}\right)=\mathbb{C P}^{2} \#\left(-\mathbb{C P}_{1}^{2}\right) \#\left(-\mathbb{C P}_{2}^{2}\right)$ such that the $H^{2}\left(\mathbb{C P}^{2}\right)$-component of $c_{1}(\mathfrak{t})$ gives a generator of $H^{2}\left(\mathbb{C P}^{2}\right)$ and $j$-th component does of $H^{2}\left(-\mathbb{C P}_{j}^{2}\right)$ for each $j=1,2$. Set

$$
X:=M_{0} \# n\left(\mathbb{C P}^{2} \# 2\left(-\mathbb{C P}^{2}\right)\right)=M_{0} \# N,
$$

where $N:=\#_{i=1}^{n} N_{i}$ and $N_{i}:=\mathbb{C P}^{2} \# 2\left(-\mathbb{C P}^{2}\right)$. Let $\varphi_{i}: M_{0} \# N_{i} \rightarrow M_{1} \# N_{i}$ be the copy of $\varphi$, and we write $\varphi_{i}: X \rightarrow M_{1} \# N$ also for the extension by the identity. Let $\mathfrak{t}$ be the isomorphism class of a $\operatorname{spin}^{c}$ structure on $N$ defined by $\mathfrak{t}=\#_{i=1}^{n} t_{i}$, where $\mathfrak{t}_{i}$ is the copy of $\mathfrak{t}_{0}$. We define the isomorphism class $\mathfrak{s}$ of a $\operatorname{spin}^{c}$ structure on $X$ by

$$
\mathfrak{s}:=\mathfrak{s}_{0} \# \mathfrak{t}
$$

Then one can easily see that $d(\mathfrak{s})=-n$. Let $f_{1,0}, \ldots, f_{n, 0} \in \operatorname{Diff}(X, \mathfrak{s})$ be the diffeomorphisms given in Theorem 3.2 in [20]. (These diffeomorphisms are written as $f_{1}, \ldots, f_{n}$ there. $)$ Each $f_{i, 0}$ is identity on $M_{0} \#\left(\#{ }_{i^{\prime} \neq i} N_{i^{\prime}}\right)$, and hence they are mutually commutative. We also note that $f_{i, 0}$ is obtained as the copy of a common diffeomorphism on $M_{0} \# \mathbb{C P}^{2} \# 2\left(-\mathbb{C P}^{2}\right)$ for any $i$. Let $f_{1,1}^{\prime}, \ldots, f_{n, 1}^{\prime} \in \operatorname{Diff}^{+}\left(M_{1} \# N\right)$ be the diffeomorphisms defined by substituting $M_{1}$ for $M_{0}$ in Theorem 3.2 in [20], and let $f_{1,1}, \ldots, f_{n, 1} \in \operatorname{Diff}^{+}(X)$ be the diffeomorphisms defined by $f_{i, 1}:=\varphi_{i}^{-1} \circ f_{i, 1}^{\prime} \circ \varphi_{i}$. Then $f_{1,1}, \ldots, f_{n, 1}$ are mutually commutative and belong to $\operatorname{Diff}(X, \mathfrak{s})$. We also note that, if $i \neq i^{\prime}$, then $f_{i, 0}$ and $f_{i^{\prime}, 1}$ are also commutative. As Ruberman [38, by the construction of $\varphi$ and $f_{i, 0}, f_{i, 1}$, we have that $f_{i, 0}$ is homotopic to $f_{i, 1}$ for each $i$. The diffeomorphism $f_{i}:=f_{i, 0} \circ f_{i, 1}^{-1} \in \operatorname{Diff}(X, \mathfrak{s})$ is therefore homotopic to the identity. In fact the result due to F. Quinn [36] implies that, more strongly, $f_{i}$ is topologically isotopic to the identity.

We here recall an invariant of $n$-tuples of commutative diffeomorphisms on an oriented closed 4-manifold defined in [20] by the author. This is a generalization of Ruberman's invariant given in 38 emerging from 1-parameter families of SeibergWitten equations. This invariant of commutative diffeomorphisms relates to $\mathbb{S W}$ as follows. For mutually commutative diffeomorphisms $f_{1}, \ldots, f_{n} \in \operatorname{Diff}(X, \mathfrak{s})$, say ones defined above, one can associate a number

$$
\mathrm{SW}\left(f_{1}, \ldots, f_{n} ; \mathfrak{s}\right) \in \mathbb{Z} \text { or } \mathbb{Z} / 2 .
$$

This is defined by counting the moduli space of families of Seiberg-Witten equations parameterized on $[0,1]^{n}$, and it can be interpreted as a counted number of a parameterized moduli space on $T^{n}$. (See Example 2.6 in [20.) This number is nothing but the counted number of $\mathcal{M}_{\sigma}$ for some inductive section $\sigma: T^{n} \rightarrow \Pi(E)$ for the bundle $X \rightarrow E \rightarrow T^{n}$ defined as the mapping torus of $f_{1}, \ldots, f_{n}$. Thus we have

$$
\mathrm{SW}\left(f_{1}, \ldots, f_{n} ; \mathfrak{s}\right)= \pm\left\langle\mathbb{S W}(E),\left[T^{n}\right]\right\rangle .
$$

(If we work over $\mathbb{Z} / 2$, of course, the plus-minus sign in the right-hand side is omitted.)

We now come back to our specific diffeomorphisms. Since $f_{i, 1}$ and $f_{i, 1}$ reverse a homology orientation, we work on $\mathbb{Z} / 2$, though $\mathrm{SW}\left(f_{1}, \ldots, f_{n} ; \mathfrak{s}\right)$ is defined over $\mathbb{Z}$. 
As Lemma 2.6 Ruberman 38, we immediately see that

$$
\begin{aligned}
\mathrm{SW}\left(f_{1}, \ldots, f_{n} ; \mathfrak{s}\right) & =\sum_{0 \leq j_{1}, \ldots, j_{n} \leq 1} \operatorname{SW}\left(f_{1, j_{1}}^{\epsilon_{1}}, \ldots, f_{n, j_{n}}^{\epsilon_{n}} ; \mathfrak{s}\right) \\
& =\sum_{0 \leq j_{1}, \ldots, j_{n} \leq 1} \operatorname{SW}\left(f_{1, j_{1}}, \ldots, f_{n, j_{n}} ; \mathfrak{s}\right)
\end{aligned}
$$

over $\mathbb{Z} / 2$, where $\epsilon_{i} \in\{1,-1\}$ is defined by $j_{i}=1 \Leftrightarrow \epsilon_{i}=-1$, and $f_{i, 0}^{1}:=f_{i, 0}$. Let $j_{1}, \ldots, j_{n} \in\{0,1\}$ and $j_{1}^{\prime}, \ldots, j_{n}^{\prime} \in\{0,1\}$. Then, if we have $\#\left\{i \mid j_{i}=0\right\}=$ $\#\left\{i \mid j_{i}^{\prime}=0\right\}$, it follows that

$$
\mathrm{SW}\left(f_{1, j_{1}}, \ldots, f_{n, j_{n}} ; \mathfrak{s}\right)=\mathrm{SW}\left(f_{1, j_{1}^{\prime}}, \ldots, f_{n, j_{n}^{\prime}} ; \mathfrak{s}\right)
$$

because of symmetry. Let us assume that $n$ can be written as $n=2^{N}$ for some $N \geq 0$. Then the binomial coefficient $\left(\begin{array}{l}n \\ k\end{array}\right)$ is even for any $k \in\{1, \ldots, n-1\}$, and it therefore follows from the equalities (57) and (58) that

$$
\mathrm{SW}\left(f_{1}, \ldots, f_{n} ; \mathfrak{s}\right)=\mathrm{SW}\left(f_{1,0}, \ldots, f_{n, 0} ; \mathfrak{s}\right)+\mathrm{SW}\left(f_{1,1}, \ldots, f_{n, 1} ; \mathfrak{s}\right)
$$

over $\mathbb{Z} / 2$. In addition, Theorem 3.2 in 20 implies that

$$
\mathrm{SW}\left(f_{1, j}, \ldots, f_{n, j} ; \mathfrak{s}\right)=\operatorname{SW}\left(M_{j}, \mathfrak{s}_{j}\right)=j+1
$$

over $\mathbb{Z} / 2$ for each $j=0,1$, and thus we have $\mathrm{SW}\left(f_{1}, \ldots, f_{n} ; \mathfrak{s}\right)=1$. We can therefore deduce that $\mathbb{S W}(E) \neq 0$ in $H^{n}\left(T^{n} ; \mathbb{Z} / 2\right)$ from (156). Thus we have:

Theorem 8.16. Let $N \geq 0$ and set $n=2^{N}$. Let $(X, \mathfrak{s})$ and $X \rightarrow E \rightarrow T^{n}$ be as above. Then $\mathbb{S W}(E) \neq 0$ holds, in particular $E$ is a non-trivial Diff $(X, \mathfrak{s})$-bundle.

Remark 8.17. We note that, in the case of $n=1$ above, since $f_{1}$ is topologically isomorphic to the identity, $X \rightarrow E \rightarrow S^{1}$ is trivial as a $\operatorname{Homeo}_{0}(X)$-bundle, and hence also as a $\operatorname{Homeo}(X, \mathfrak{s})$-bundle. Here $\operatorname{Homeo}_{0}(X)$ is the identity component of the whole group of orientation preserving homeomorphisms on $X$.

Remark 8.18. Although a result of F. Quinn [36] gives topological isotopies between $f_{1}, \ldots, f_{n}$ and the identity, the supports of the topological isotopies are not disjoint. The topological isotopies are therefore not mutually commutative in general, and we cannot conclude that $E$ is trivial as a $\operatorname{Homeo}(X, \mathfrak{s})$-bundle unless $n=1$ from [36]. The author does not know whether $E$ is trivial as $\operatorname{Homeo}(X, \mathfrak{s})$-bundle when $n>1$.

Example 8.19. If we substitute $K 3$ for $M_{0}$ in the above argument, we have

$$
X \cong(n+3) \mathbb{C P}^{2} \#(2 n+19)\left(-\mathbb{C P}^{2}\right) .
$$

In this case we can take the (isomorphism class of a) $\operatorname{spin}^{c}$ structure coming from the complex structure of $K 3$ as $\mathfrak{s}_{0}$. Let $\mathfrak{s}$ be the (isomorphism class of a) $\operatorname{spin}^{c}$ structure on $X$ defined by (54). From the diffeomorphism (60) and Theorem 8.16 if $n$ can be written as $n=2^{N}$ for some $N \geq 0$, we get a bundle

$$
(n+3) \mathbb{C P}^{2} \#(2 n+19)\left(-\mathbb{C P}^{2}\right) \rightarrow E \rightarrow T^{n}
$$

with structure group $\operatorname{Diff}(X, \mathfrak{s})$ satisfying $\mathbb{S W}(E) \neq 0$. This is therefore non-trivial as a $\operatorname{Diff}(X, \mathfrak{s})$-bundle, and trivial as a $\operatorname{Homeo}(X, \mathfrak{s})$-bundle if $n=1$, explained in Remark 8.17 
We here note that, to show only the non-triviality of our characteristic classes, one can directly use author's result [20]. As explained in (59), Theorem 3.2 in [20] gives diffeomorphisms whose invariant explained in (55) is non-trivial over $\mathbb{Z} / 2$, and thus we obtain:

Theorem 8.20. Let $M$ be an oriented closed smooth 4 -manifold with $b^{+}(M) \geq 2$ and $\mathfrak{s}_{0}$ be the isomorphism class of a spin ${ }^{c}$ structure on $M$ such that the SeibergWitten invariant of $\left(M, \mathfrak{s}_{0}\right)$ is an odd number. Let $n$ be a positive integer. Define $N=n \mathbb{C P}^{2} \# m\left(-\mathbb{C P}^{2}\right)=\#_{i=1}^{n} \mathbb{C P}_{i}^{2} \#\left(\#_{j=1}^{m}\left(-\mathbb{C P}_{j}^{2}\right)\right)$ for some $m \geq 2 n$ and $\mathfrak{t}$ is the isomorphism class of a spinc structure on $N$ such that the $i$-th component of $c_{1}(\mathfrak{t})$ gives a generator of $H^{2}\left(\mathbb{C P}_{i}^{2}\right)$ and $j$-th component does of $H^{2}\left(-\mathbb{C P}_{j}^{2}\right)$. Then, for the pair defined by

$$
(X, \mathfrak{s}):=\left(M \# N, \mathfrak{s}_{0} \# \mathfrak{t}\right)
$$

we have

$$
\mathbb{S W}(X, \mathfrak{s}) \neq 0 \text { in } H^{n}(B \operatorname{Diff}(X, \mathfrak{s}) ; \mathbb{Z} / 2) .
$$

Proof. Let $X \rightarrow E \rightarrow T^{n}$ be the mapping torus of the diffeomorphisms $f_{1}, \ldots, f_{n}$ given in Theorem 3.2 in [20]. Then (56) and Theorem 3.2 in [20] imply that $\left\langle\mathbb{S W}(E),\left[T^{n}\right]\right\rangle=\mathrm{SW}\left(f_{1}, \ldots, f_{n} ; \mathfrak{s}\right) \neq 0$ in $\mathbb{Z} / 2$. The assertion in the theorem therefore follows from functoriality (Theorem 6.6).

8.4. Behavior under the composition 2. We here discuss a spin analogue of Subsection 8.3. we consider $S^{2} \times S^{2}$ in this subsection instead of $\mathbb{C P}^{2} \# 2\left(-\mathbb{C P}^{2}\right)$ in Subsection 8.3. Fix $n>0$ and let $M_{0}$ be an oriented closed spin smooth 4-manifold such that $\operatorname{sign}\left(M_{0}\right)=0$ (hence $M_{0}$ has even $\left.b_{2}\right)$ and $b^{+}\left(M_{0}\right) \geq 2$, and let $\mathfrak{s}_{0}$ be the isomorpshim class of a $\operatorname{spin}^{c}$ structure on $M_{0}$. Assume that the Seiberg-Witten invariant of $\left(M, \mathfrak{s}_{0}\right)$ is an odd number and that $M_{0} \# S^{2} \times S^{2}$ is diffeomorphic to $M_{1} \# S^{2} \times S^{2}$, where $M_{1}=\frac{b_{2}\left(M_{0}\right)}{2}\left(S^{2} \times S^{2}\right)$. Set

$$
X:=M_{0} \# n\left(S^{2} \times S^{2}\right) .
$$

Let $\mathfrak{s}$ be the isomorphism class of a $\operatorname{spin}^{c}$ structure on $X$ defined by $\mathfrak{s}:=\mathfrak{s}_{0} \# \mathfrak{t}$, where $\mathfrak{t}$ is the isomorphism class of $\operatorname{spin}^{c}$ structure coming from a spin structure on $n\left(S^{2} \times S^{2}\right)$. Recall that $f_{1, j}, \ldots, f_{n, j}$ in Subsection 8.3 are given as the extension of the $n$-tuple of diffeomorphisms on $n\left(\mathbb{C P}^{2} \# 2\left(-\mathbb{C P}^{2}\right)\right)$ given in [20] by the identity on $M_{i}$. If we use the diffeomorphisms $f_{1}, \ldots, f_{n}$ on $n\left(S^{2} \times S^{2}\right)$ in Subsection 8.2 instead of this $n$-tuple of diffeomorphisms on $n\left(\mathbb{C P}^{2} \# 2\left(-\mathbb{C P}^{2}\right)\right)$, we get commutative diffeomorphisms $f_{1, j}, \ldots, f_{n, j} \in \operatorname{Diff}(X, \mathfrak{s})$ for $j=0,1$. In this subsection we use the notation $f_{1}, \ldots, f_{n}$ for the diffeomorphisms defined as $f_{i, 0} \circ f_{i, 1}^{-1}$. Each $f_{i}$ is topologically isotopic to the identity as in Subsection 8.3. Let $X \rightarrow E \rightarrow T^{n}$ be the mapping torus of $f_{1}, \ldots, f_{n}$. Instead of Theorem 3.2 in [20] used in Subsection 8.3. we can use calculations given in Subsection 8.2. More precisely, in the proof of Theorem 8.9, we have shown that

$$
\mathrm{SW}\left(f_{1, j}, \ldots, f_{n, j} ; \mathfrak{s}\right)=j+1
$$

over $\mathbb{Z} / 2$. We can therefore deduce the following theorem by the same argument of Subsection 8.3 .

Theorem 8.21. Let $N \geq 0$ and set $n=2^{N}$. Let $(X, \mathfrak{s})$ and $X \rightarrow E \rightarrow T^{n}$ be as above. Then $\mathbb{S W W}(E) \neq 0$ holds, in particular $E$ is a non-trivial $\operatorname{Diff}(X, \mathfrak{s})$-bundle. 
Remark 8.22. By the same reason described in Remark 8.17, in the case of $n=1$, the bundle $X \rightarrow E \rightarrow S^{1}$ is trivial as a $\operatorname{Homeo}(X, \mathfrak{s})$-bundle.

Example 8.23. We here use the 4-manifold $X(p, q)$ in Example 8.11, We take $p, q$ so that $X(p, q) \# S^{2} \times S^{2} \cong m\left(S^{2} \times S^{2}\right)$ for some $m>0$. In the above argument, let us substitute $X(p, q)$ for $M_{0}$, and as $\mathfrak{s}_{0}$ take the (isomorphism class of a) $\operatorname{spin}^{c}$ structure coming from the symplectic structure of $X(p, q)$. From the dissolving of $X(p, q) \# S^{2} \times S^{2}$ and Theorem 8.21 , if $n$ can be written as $n=2^{N}$ for some $N \geq 0$, we get a bundle

$$
k\left(S^{2} \times S^{2}\right) \rightarrow E \rightarrow T^{n}
$$

with structure group $\operatorname{Diff}(X, \mathfrak{s})$ satisfying $\mathbb{S W}(E) \neq 0$, where $k=m+n-1$. This is therefore non-trivial as a $\operatorname{Diff}(X, \mathfrak{s})$-bundle, and trivial as a $\operatorname{Homeo}(X, \mathfrak{s})$-bundle if $n=1$.

Remark 8.24. As remarked in Remark 8.12, it seems difficult to determine $k$ 's in Example 8.23 .

8.5. Ruberman's calculation and $\mathbb{D}$. We mention a calculation of $\mathbb{D}$ obtained from Ruberman's one given in [38. In Theorem 3.1 in [38, he has constructed a diffeomorphism preserving the isomorphism class of an $S O(3)$-bundle on some 4-manifold $X$ such that his invariant of the diffeomorphism based on a 1-parameter family of ASD equations does not vanish. This invariant coincides with the counted number of the parametrized moduli space of ASD equations for the family $X \rightarrow$ $E \rightarrow S^{1}$ defined as the mapping torus, as in Subsections 8.3 and 8.4 Thus we have:

Theorem 8.25. Let $M$ be an oriented closed smooth 4-manifold with $b^{+}(M) \geq 2$ and $\mathfrak{P}_{0}$ be the isomorphism class of an $S O(3)$-bundle on $M$ such that the formal dimension for $\mathfrak{P}_{0}$ is zero and $w_{2}\left(\mathfrak{P}_{0}\right) \neq 0$, and that the Donaldson invariant of $\left(M, \mathfrak{P}_{0}\right)$ does not vanish (over $\left.\mathbb{Z}\right)$. Set

$$
X=M \# \mathbb{C P}^{2} \# 2\left(-\mathbb{C P}^{2}\right) .
$$

Let $L \rightarrow \mathbb{C P}^{2} \# 2\left(-\mathbb{C P}^{2}\right)$ be the complex line bundle such that the $H^{2}\left(\mathbb{C P}^{2}\right)$-component of $c_{1}(L)$ is a generator of $H^{2}\left(\mathbb{C P}^{2}\right)$, and similarly the $H^{2}\left(-\mathbb{C P}_{j}^{2}\right)$-component of $c_{1}(L)$ is a generator of $H^{2}\left(-\mathbb{C P}_{j}^{2}\right)$ for $j=1,2$. We define $\mathfrak{P}$ as the isomorphism class of the $S O(3)$-bundle obtained by gluing (a representative of) $\mathfrak{P}_{0}$ with $L \oplus \underline{\mathbb{R}}$, where $\mathbb{R}$ is the trivial real line bundle. Then, for a fixed a homology orientation $\mathcal{O}$ on $X$, we have

$$
\mathbb{D}(X, \mathfrak{P}, \mathcal{O}) \neq 0 \text { in } H^{1}(B \operatorname{Diff}(X, \mathfrak{P}, \mathcal{O}) ; \mathbb{Z}) .
$$

Proof. Theorem 3.1 in Ruberman [38] asserts that there is a diffeomorphism on $X$ such that it preserves $\mathfrak{P}$ and $\mathcal{O}$ and that his invariant of the diffeomorphism coincides with the -4 times the Donaldson invariant of $\left(M, \mathfrak{P}_{0}\right)$. The assertion in the theorem therefore follows as in the proof of Theorem 8.20 .

Remark 8.26. At this stage the author does not have a non-trivial example of $\mathbb{D}$ for more higher degree $n>1$. In the argument of Section 8 , the difference on computability for $\mathbb{S W}$ and for $\mathbb{D}$ arises from the difference between the structure of wall in both theories: the wall in Donaldson theory is more complicated rather than that of Seiberg-Witten theory. To generalize Theorem 8.25 for $n>0$, one has to study the structure of the wall in Donaldson theory for $b^{+}>0$. 


\section{CONCLUding REMARKS}

Finally, we note some related work which we have not mention and also note further potential developments of our characteristic classes $\mathbb{D}$ and $\mathbb{S W}$.

Remark 9.1. P. Kronheimer 22 has defined an invariant for families of SeibergWitten equations. More precisely, he has considered a family parameterized on a singular chain in the space of perturbations such that the parameterized moduli space vanishes on the boundary. Namely, his invariant is a version of relative invariants for families. In symplectic geometry, several people have considered relative Gromov-Witten invariants using Kronheimer's work. See O. Buşe [6] and T. Nishinou [34. In our context, we can define a similar relative version of our characteristic classes as follows. Let $X \rightarrow E \rightarrow B$ a bundle of an oriented closed smooth 4-manifold $X$ on a CW complex $B$. Let us choose either the ASD setting or the SW setting, and suppose that the formal dimension is $-n$ for $n>0$. Fix an inductive section $\sigma:\left.B^{(n)} \rightarrow \Pi(E)\right|_{B^{(n)}}$. Let $B^{\prime} \subset B$ be a subspace of $B$ such that the parameterized moduli space with respect to $\sigma$ vanishes on $B^{\prime}$. Set $\tau:=\left.\sigma\right|_{B^{\prime} \cap B^{(n)}}$. Then we can define $\mathbb{A}\left(E, B^{\prime}, \tau\right) \in H^{n}\left(B, B^{\prime}\right)$ as $\mathbb{A}(E)$. The relative cohomology class $\mathbb{A}\left(E, B^{\prime}, \tau\right)$ depends only on $E, B^{\prime}$, and $\tau$, i.e., for another inductive section $\sigma^{\prime}$ satisfying $\left.\sigma^{\prime}\right|_{B^{\prime} \cap B^{(n)}}=\tau$, we can get the same cohomology class.

Remark 9.2. M. Szymik [41] has considered a family version of the Bauer-Furuta invariant [5. As described in the introduction, one of the big differences between his setting and ours is the structure group of families. The author expects that a Bauer-Furuta-type refinement can be also considered in our setting: families with structure group $\operatorname{Diff}(X, \mathfrak{s})$ or $\operatorname{Diff}(X, \mathfrak{s}, \mathcal{O})$. To establish such a refinement in full generality, we need some stacks, which will be discussed in a subsequent paper. (See also Remark 6.11)

Remark 9.3. A possibility of a generalization of our theory is to construct a version of Floer theory for $\mathbb{D}$ and $\mathbb{S W}$. If we try to do it, we are faced with a serious analytic problem: if we consider a family of 3-manifolds, the differential of the Floer chain complexes varies, and in addition, critical points also do in the Seiberg-Witten situation. One might hope that we shall overcome such a problem by introducing techniques developed in the context of symplectic geometry. See, for example, K. Fukaya [13] and M. Abouzaid [1,2].

\section{REFERENCES}

[1] Mohammed Abouzaid, Family Floer cohomology and mirror symmetry, Proceedings of the International Congress of Mathematicians 2014, available at arXiv:1404.2659

[2] ㄴ The family Floer functor is faithful, J. Eur. Math. Soc. (JEMS) 19 (2017), no. 7, 2139-2217. MR3656481

[3] M. F. Atiyah, V. K. Patodi, and I. M. Singer, Spectral asymmetry and Riemannian geometry. I, Math. Proc. Cambridge Philos. Soc. 77 (1975), 43-69. MR0397797

[4] M. F. Atiyah and I. M. Singer, The index of elliptic operators. IV, Ann. of Math. (2) 93 (1971), 119-138. MR0279833

[5] Stefan Bauer and Mikio Furuta, A stable cohomotopy refinement of Seiberg-Witten invariants. I, Invent. Math. 155 (2004), no. 1, 1-19, DOI 10.1007/s00222-003-0288-5. MR2025298

[6] Olguţa Buşe, Relative family Gromov-Witten invariants and symplectomorphisms, Pacific J. Math. 218 (2005), no. 2, 315-341. MR2218350

[7] S. K. Donaldson, Yang-Mills invariants of four-manifolds, Geometry of low-dimensional manifolds, 1 (Durham, 1989), London Math. Soc. Lecture Note Ser., vol. 150, Cambridge Univ. Press, Cambridge, 1990, pp. 5-40. MR1171888 
[8] _ Polynomial invariants for smooth four-manifolds, Topology 29 (1990), no. 3, 257315. MR1066174

[9] _ The Seiberg-Witten equations and 4-manifold topology, Bull. Amer. Math. Soc. (N.S.) 33 (1996), no. 1, 45-70. MR1339810

[10] _ Floer homology groups in Yang-Mills theory, Cambridge Tracts in Mathematics, vol. 147, Cambridge University Press, Cambridge, 2002. With the assistance of M. Furuta and D. Kotschick. MR1883043

[11] Paul M. N. Feehan and Thomas G. Leness, SO(3) monopoles, level-one Seiberg-Witten moduli spaces, and Witten's conjecture in low degrees, Proceedings of the 1999 Georgia Topology Conference (Athens, GA), 2002, pp. 221-326. MR1936209

[12] _ An SO(3)-monopole cobordism formula relating Donaldson and Seiberg-Witten invariants, available at arXiv:math/0203047.

[13] Kenji Fukaya, Floer homology for families - a progress report, Integrable systems, topology, and physics (Tokyo, 2000), Contemp. Math., vol. 309, Amer. Math. Soc., Providence, RI, 2002, pp. 33-68. MR1953352

[14] Mikio Furuta, A remark on a fixed point of finite group action on $S^{4}$, Topology 28 (1989), no. 1, 35-38. MR991097

[15] B. Hanke, D. Kotschick, and J. Wehrheim, Dissolving four-manifolds and positive scalar curvature, Math. Z. 245 (2003), no. 3, 545-555, DOI 10.1007/s00209-003-0553-8. MR2021570

[16] Allen Hatcher, Algebraic topology, Cambridge University Press, Cambridge, 2002. MR1867354

[17] Michel A. Kervaire, Relative characteristic classes, Amer. J. Math. 79 (1957), 517-558. MR0090051

[18] H. Konno, Bounds on genus and configurations of embedded surfaces in 4-manifolds, J. Topol. 9 (2016), no. 4, 1130-1152, DOI 10.1112/jtopol/jtw021.

[19] — A cohomological Seiberg-Witten invariant emerging from the adjunction inequality, available at arXiv: 1704.05859

[20] _ Positive scalar curvature and higher-dimensional families of Seiberg-Witten equations, available at arXiv:1707.08974

[21] Andreas Kriegl and Peter W. Michor, The convenient setting of global analysis, Mathematical Surveys and Monographs, vol. 53, American Mathematical Society, Providence, RI, 1997. MR1471480

[22] P. Kronheimer, Some non-trivial families of symplectic structures, preprint.

[23] Serge Lang, Differential and Riemannian manifolds, 3rd ed., Graduate Texts in Mathematics, vol. 160, Springer-Verlag, New York, 1995. MR1335233 (96d:53001)

[24] Hông-Vân Lê and Kaoru Ono, Parameterized Gromov-Witten invariants and topology of symplectomorphism groups, Groups of diffeomorphisms, Adv. Stud. Pure Math., vol. 52, Math. Soc. Japan, Tokyo, 2008, pp. 51-75. MR2509705

[25] Tian-Jun Li and Ai-Ko Liu, Family Seiberg-Witten invariants and wall crossing formulas, Comm. Anal. Geom. 9 (2001), no. 4, 777-823. MR1868921 (2002k:57074)

[26] J. P. May, A concise course in algebraic topology, Chicago Lectures in Mathematics, University of Chicago Press, Chicago, IL, 1999. MR1702278

[27] Dusa McDuff, The symplectomorphism group of a blow up, Geom. Dedicata 132 (2008), 1-29. MR2396906

[28] Richard B. Melrose, The Atiyah-Patodi-Singer index theorem, Research Notes in Mathematics, vol. 4, A K Peters, Ltd., Wellesley, MA, 1993. MR1348401

[29] Edward Y. Miller, The homology of the mapping class group, J. Differential Geom. 24 (1986), no. 1, 1-14. MR857372

[30] Shigeyuki Morita, Characteristic classes of surface bundles, Invent. Math. 90 (1987), no. 3, 551-577. MR914849

[31] David Mumford, Towards an enumerative geometry of the moduli space of curves, Arithmetic and geometry, Vol. II, Progr. Math., vol. 36, Birkhäuser Boston, Boston, MA, 1983, pp. 271328. MR717614

[32] Nobuhiro Nakamura, The Seiberg-Witten equations for families and diffeomorphisms of 4-manifolds, Asian J. Math. 7 (2003), no. 1, 133-138, DOI 10.4310/AJM.2003.v7.n1.a8. MR2015245

[33] Smoothability of $\mathbb{Z} \times \mathbb{Z}$-actions on 4-manifolds, Proc. Amer. Math. Soc. 138 (2010), no. 8, 2973-2978, DOI 10.1090/S0002-9939-10-10413-4. MR2644908 
HOKUTO KONNO

[34] Takeo Nishinou, Some nontrivial homology classes on the space of symplectic forms, J. Math. Kyoto Univ. 42 (2002), no. 3, 599-606. MR1967225

[35] Jongil Park, The geography of Spin symplectic 4-manifolds, Math. Z. 240 (2002), no. 2, 405-421, DOI 10.1007/s002090100390. MR1900318

[36] Frank Quinn, Isotopy of 4-manifolds, J. Differential Geom. 24 (1986), no. 3, 343-372. MR868975

[37] Yongbin Ruan, Virtual neighborhoods and the monopole equations, Topics in symplectic 4manifolds (Irvine, CA, 1996), First Int. Press Lect. Ser., I, Int. Press, Cambridge, MA, 1998, pp. 101-116. MR1635698 (2000e:57054)

[38] Daniel Ruberman, An obstruction to smooth isotopy in dimension 4, Math. Res. Lett. 5 (1998), no. 6, 743-758, DOI 10.4310/MRL.1998.v5.n6.a5. MR1671187 (2000c:57061)

[39] _ A polynomial invariant of diffeomorphisms of 4-manifolds, Proceedings of the Kirbyfest (Berkeley, CA, 1998), Geom. Topol. Monogr., vol. 2, Geom. Topol. Publ., Coventry, 1999, pp. 473-488 (electronic), DOI 10.2140/gtm.1999.2.473. MR1734421 (2001b:57073)

[40] _ Positive scalar curvature, diffeomorphisms and the Seiberg-Witten invariants, Geom. Topol. 5 (2001), 895-924 (electronic), DOI 10.2140/gt.2001.5.895. MR1874146 (2002k:57076)

[41] Markus Szymik, Characteristic cohomotopy classes for families of 4-manifolds, Forum Math. 22 (2010), no. 3, 509-523. MR2652709

[42] C. T. C. Wall, On simply-connected 4-manifolds, J. London Math. Soc. 39 (1964), 141-149, DOI 10.1112/jlms/s1-39.1.141. MR0163324

[43] Edward Witten, Monopoles and four-manifolds, Math. Res. Lett. 1 (1994), no. 6, 769-796, DOI 10.4310/MRL.1994.v1.n6.a13. MR1306021

Graduate School of Mathematical Sciences, the University of Tokyo, 3-8-1 Komaba, Meguro, TOKYO 153-8914, JAPAN

E-mail address: hkonno@ms.u-tokyo.ac.jp 